\title{
Teoria e prassi delle transizioni costituzionali e del consolidamento democratico agli inizi del nuovo millennio
}

Luca Mezzetti

\section{Introduzione}

Agli albori del XXI secolo, al costituzionalismo liberale sembra doversi riconoscere una pluralità di successi su vari versanti. Da un lato, infatti, il modello liberale ha pienamente compiuto (ovvero appare in procinto di compiere) la missione storicamente affidatagli ai fini del definitivo consolidamento della democrazia in un numero non irrilevante di ordinamenti, che tendono a coincidere con le esperienze costituzionali dell'Europa occidentale e dei Paesi di matrice anglosassone dell'area nordamericana (Stati Uniti e Canada) e pacifica (Australia e Nuova Zelanda), dovendosi comprendere in quest'ultima anche il caso del Giappone. Il successo del costituzionalismo liberale può dirsi, nei menzionati ordinamenti, pieno.

D'altro lato, non può mancarsi di osservare come il modello liberale abbia comunque rappresentato, nel contesto delle diverse ondate di democratizzazione verificatesi dopo il 1945, un punto di riferimento per un elevato numero di ordinamenti interessati dal processo di decolonizzazione $\mathrm{e}$, più recentemente, dal mutamento della forma di stato e di governo conseguente al crollo dei regimi comunisti (è il caso dei Paesi dell'Europa centro-orientale e dell' area ex-sovietica) ovvero dal tentativo di democratizzazione e di modernizzazione delle strutture costituzionali e dei sistemi politici derivati dalle esperienza post-coloniale (è il caso dei Paesi africani e asiatici). Il successo del costituzionalismo liberale si è rivelato, in questo secondo insieme di ordinamenti, solo parziale.

In realtà, con riferimento alle funzioni e alle attitudini del modello costituzionale liberale, si può individuare un denominatore comune ai 
due insiemi sopra considerati : la capacità del modello medesimo di atteggiarsi quale strumento di resistenza e di reazione nelle fasi di deligittimazione politica dei miti antidemocratici (Habermas), nonchè quale strumento di consolidamento delle conquiste democratiche nelle fasi successive. Tali fasi hanno coinciso, per le esperienze costituzionali occidentali, con la sconfitta degli autoritarismi nel 1945 da una parte e con la edificazione dello Stato sociale di diritto dall'altra; per le esperienze costituzionali dei Paesi di recente (o riacquisita) indipendenza o in via di sviluppo (dovendosi intendere comprensiva tale categoria degli ordinamenti dell'Europa centro-orientale), con la decolonizzazione e con il varo della modernizzazione dell'assetto istituzionale ed economico ovvero, nel caso degli ordinamenti ex-socialisti, con la rifondazione del medesimo. In tal senso il «secolo breve», presentato da autorevole dottrina storicistica (Hobsbawm) quale traiettoria che si dispiega senza soluzioni di continuità dal 1914 al 1989, appare in realtà caratterizzato da una profonda, positiva cesura -la vittoria delle democrazie contro gli autoritarismi- tale da configurarsi quale evento capace di privare "di qualsiasi legittimazione chi da allora non avesse reso omaggio (anche soltanto verbalmente, anche soltanto con riferimento alla lettera) allo spirito universalistico dell'illuminismo politico" (Habermas).

La affermazione solo relativa che il costituzionalismo liberale ha registrato può spiegarsi con una serie di ragioni, non riduttivamente riconducibili alla difficoltà di innestare e trapiantare il modello liberale in seno ad ordinamenti pronti a recepirne solo alcuni contenuti e principi essenziali, rigettandone invece altri. Accanto a motivazioni o cause di ordine giuridico e culturale, infatti, altre ne emergono di tipo economico e strutturale, derivanti da fattori di matrice esogena ed endogena, procedenti dalle peculiarità della società civile, identificabili con ragioni di tipo religioso, riconducibili al ruolo dei militari e delle forze armate. E' alla luce di tali considerazioni che si comprende come una pluralità di ordinamenti sia tuttora chiamato a misurarsi con una doppia sfida : la risoluzione dei problemi dello sviluppo, la modernizzazione della propria struttura istituzionale e le questioni poste dalla globalizzazione e dalla internazionalizzazione.

I compiti connessi a tali questioni si sono spesso rivelati (e tuttora si rivelano), in una pluralità di ipotesi, un fardello di entità qualitativa $e$ quantitativa eccessivamente oneroso per le gracili strutture di ordinamenti che, faticosamente superata la fase della transizione costituzionale, si 
trovano ad affrontare la non meno delicata fase del consolidamento democratico. In tal senso, particolarmente evocativa appare l'immagine tratteggiata da autorevole dottrina politologica (Huntington) per sottolineare la natura (almeno potenzialmente) anfidrome dei processi di democratizzazione : ad ogni ondata di democratizzazioni -che rappresenta per alcune esperienze costituzionali un momento di approdo susseguente ad una perigliosa navigazione nelle acque della transizione istituzionale - corrisponde una ondata di ritorno (reverse wave) che ne trascina altre, sfinite e spossate dal tentativo di assolvere tale duplice (immane) compito, verso il limbo, giuridico e politico, delle democrazie incerte o verso il baratro del regresso autoritario.

L'analisi comparatistica che intenda penetrare tale complesso di ragioni non può in ogni caso prescindere dall'apporto sia delle scienze giuridiche sia delle scienze storico-politiche, da utilizzarsi secondo un metodo di feconda osmosi, compenetrazione e sussidiarietà.

Tale approccio alle istituzioni costituzionali non è -come è stato autorevolmente notato (Barbera) - consueto nella letteratura, essendo finora prevalse le analisi politiche o la mera comparazione fra istituti giuridici. E' peraltro significativo come, a fronte della attuale diffusa attenzione per i temi della globalizzazione e della necessità di fornire adeguate risposte alle sfide poste al costituzionalismo dal contesto internazionalizzato nel suo complesso (definizione di nuovi ruoli e attualità delle funzioni del costituzionalismo, rimodulazione dell'assetto e delle funzioni dello stato nazionale ; definizione di una nuova e diversa valenza del concetto e del contenuto della sovranità popolare), su altro versante la dottrina si interroghi sulle funzionalità del costituzionalismo in sede di consolidamento democratico in una pluralità di ordinamenti e di esperienze costituzionali che si trovano tuttora a metà del guado della propria transizione e recano indici di incertezza e incompiutezza quanto alla piena realizzazione dell'opera di convergenza fra dato costituzionale formale e garanzia della effettività dei principi e degli strumenti codificati in seno al medesimo.

In tal senso, l'opera del comparatista è resa particolarmente ardua dalla necessità di individuare in via preliminare quale tertium comparationis una nozione minimale di democrazia funzionale in via propedeutica alla non facile comparabilità fra sistemi giuridici spesso profondamente diversi, nonché dal dovere di rendere oggetto di indagine una pluralità di fattori emergenti da ambiti diversi da quello strettamente giuridico e capaci di 
incidere ampiamente sulla caratterizzazione descrittiva e contenutistica delle due fasi dellae transizioni costituzionali e del consolidamento democratico.

\section{Parte prima. Le categorie delle transizioni politiche e costituzionali}

\section{La transizione}

Il vocabolo «transizione» intende indicare un passaggio di condizione.

La definizione che ne forniscono O'Donnell e Schmitter (1986) ${ }^{1}$ come "'intervallo che si estende tra un regime politico e un altro", è la più lata possibile. Finisce per abbracciare tutti i possibili cambiamenti del sistema politico ${ }^{2}$ quanto ad approdo (che spazia in una gamma che individua la democrazia e l'autocrazia come suoi estremi) e quanto a modalità (anch'esse suscettibili di essere più o meno democratiche). Spostandosi su una dimensione temporale (l'«intervallo"), tale accezione va oltre il raffronto tra status di partenza e status di approdo (vale a dire l'identificazione del "cambiamento"), per conglobare il complesso di fattori, accadimenti, attori e sentimenti che si agita nello spazio temporale considerato.

La transizione nel tipo di regime implica il movimento verso «altro di differente» e ha termine quando la "anormalità» cessa di essere la caratteristica centrale della vita politica. La «normalità» è costituita dall'insieme di regole (che prende il nome di «regime») entro le quali si muovono gli attori politici (per accedere al governo, per confrontarsi, per prendere decisioni, per escludere altri dalla contesa).

Di tutti i possibili regime changes, la battigia della terza ondata di democratizzazioni ha messo a fuoco la "transizione alla democrazia», tan-

1 G. O’Donnell e P. C. Schmitter, "Definición de algunos conceptos (y exposición de algunas premisas)", in: O'Donnell, G., Schmitter y P. C., Whitehead, L., Transiciones desde un gobierno autoritario, versione in castigliano supervisionata da Oszlak O., Tomo IV, Conclusiones tentativas sobre las democracias inciertas, Barcelona-Buenos Aires, 1988 (1994), pp. 19-20 e 118, già in: Transitions from Authoritarian Rule, Tomo IV, Tentative Conclusions about Uncertain Democracies, Baltimore-London, 1986.

2 Per la non perfetta mutua adattabilità dei concetti di «regime» e "forma di governo" sia consentito il rinvio a Mezzetti L., Le democrazie incerte. Transizioni costituzionali e consolidamento della democrazia in Europa orientale, Africa, America Latina, Asia, Torino, 2000, p. 150. 
to da supporre la nascita (e breve vita?) di un'apposita disciplina: Transitology. ${ }^{3}$

L'evoluzione di un ordinamento che si affranca da un regime non democratico per produrre una democrazia diviene allora «la» transizione, in via antonomastica. ${ }^{4}$

La dimensione perimetrale è segnata da uno sgretolamento, quantomeno significativo, del regime autocratico, quale punto di partenza, ${ }^{5}$ e dall'insediarsi di un governo democratico elettivo, quale estremo finale.

L'incertezza, ${ }^{6}$ carattere dominante ai limiti, connota tutto il dispiegarsi dell'arco temporale della transizione. La transizione alla democrazia non è processo lineare o razionale: notevole è la plasticità e finanche l'indefinitezza delle identità politiche, troppi sono i dubbi che pervadono

3 Vedi P. C. Schmitter, «Transitology: The Sciences or the Art of Democratization?", in: Tulchin J. \& Romero B. (a cura di), The Consolidation of Democracy in Latin America, Boulder [Co], 1995, pp. 11-41; Schmitter P. e Karl T., "The Conceptual Travels of Transitologists and Consolidologists: How Far to the East Should They Attempt to Go?", in: Slavic Review, No 53, 1994, pp. 173-185; Bunce V., "Should Transitologists Be Grounded?", in: Slavic Review, No 54 1994, pp. 111-125; Mansilla H. C. F., "Los limites de la democracia contemporánea y de las teorias de la transición», in: NS, No 166, 2000, pp. 62-75. Diamond L., con riferimento a Rustow - come precursore-, ad O'Donnell, Schmitter e Karl, parla di "Scuola della transizione", in: El final de la tercera ola y el futuro global de la Democracia, in: López E., Mainwaring S. (a cura di), Democracia: discusiones y nuevas aproximaciones, Buenos Aires, 2000, p. 80.

Già O' Donnell e Schmitter avvertivano l'inadeguatezza della scienza sociale classica per spiegare il fenomeno «transizione»: cfr. "Introdución de la incertidumbre», in: Transiciones desde un gobierno autoritario, Tomo IV, op. cit., p. 17.

4 Cfr., per tutti, Garretón M. A., "Democracia, transición y consolidación, un esquema general", in: Reconstruir la política. Transición y Consolidación Democrática en Chile, Santiago, 1987, p. 28 e 36.

5 Dissente Mainwaring: "[ऐे] un errore presumere che il deterioramento sia sempre una condizione per iniziare il processo di extrication. Invero, alcuni regimi autoritari intraprendono un processo di liberalizzazione in un momento di forza piutrosto che da una posizione di debolezza». L'affermazione, in sé parecchio circoscritta quanto campo d'applicazione, prova troppo e induce a sovrapporre e confondere i concetti di transizione e liberalizzazione. Cfr. Mainwaring S., "Transitions to Democracy and Democratic Consolidation: Theoretical and Comparative Issues", in: Mainwaring S., O'Donnell G. \& Valenzuela A., (a cura di) Issues in Democratic Consolidations: The New South American Democracies in Comparative Perspective, Notre Dame [Ind], 1992.

6 Il tratto è identificato da O'Donnell G. e Schmitter P. C., "Introdución de la incertidumbre», in: Transiciones desde un gobierno autoritario, Tomo IV, op. cit., cap. 1 . 
ogni soggetto coinvolto, frequente la commistione di motivi ed interessi, non esclusa è la possibilità che alcuni fattori necessari o sufficienti per fuoriuscire dal regime autoritario cambino di significato o di segno. ${ }^{7}$ A determinare il risultato finale concorrono eventi inattesi ("fortuna»), decisioni talora repentine e audaci, il talento di taluno («virtù») e la diffidenza che nutrono gli uni verso gli altri. ${ }^{8}$ Altro carattere fondamentale è l'indefinitezza delle regole del gioco politico nel dipanarsi della transizione : emergono in flusso incessante, dopo essere state, in buona misura, oggetto di aspra contesa. Tali regole affioranti definiranno in larga misura attori e mezzi che legittimamente occuperanno l'arena politica.

L'incertidumbre si declina, poi, in campo sociale e, in ultima istanza, in latente reversibilità del processo. ${ }^{9}$

O'Donnell e Schmitter (1986) utilizzano la metafora di una partita su scacchiera multidimensionale: una partita "veloce e tumultuosa», in cui la scacchiera ha plurime superfici, i giocatori sono in numero indeterminato ${ }^{10}$ e dotati di scarso fair-play, le regole stesse sono mutabili, ${ }^{11}$ specie mediante accordo, e in cui si registra una indefinitezza di vincitori e perdenti.

7 Si tratta di un allargamento del principio delineato da Fritz Stern nel 1966, operato da O'Donnell e Schmitter.

8 Lindeterminatezza circonda tutti i partecipanti alla transizione, quanto alla loro interazione immediata, come, considerata la proiezione in futuro del loro rapportarsi, per le conseguenze a medio-lungo termine.

9 Il procedimento di instaurazione di una democrazia è un processo di istituzionalizzazione dell'incertezza, di assoggettamento di tutti gli interessi all'incertezza [...]. Democrazia significa che ogni gruppo [ed ogni individuo] deve sottoporre i propri interessi all'irresolutezza. Cfr. Przeworski A., "Some Problems in the Study of the Transition to Democracy", in: O'Donnell G., Schmitter P. S. \& Whitehead L., Transition from Authoritarian Rule, tomo III, Comparative Perspectives, Baltimore-London, 1986, pp. 47-63.

$10 \mathrm{La}$ "resurrezione della società civile» dovrebbe garantire l'apporto di nuovi , più eterogenei e stimolanti giocatori.

In proposito, si veda la critica di Whitehead: come possono i padroni degli scacchi [chessmaster] o autori del gioco controllare e orchestrare i movimenti di tutti gli scacchisti [chessplayer]? Vedi Whitehead L., The Drama of Democratization, Juan March Institute, Madrid, Working Paper No 105, dicembre, 1997.

11 Pur in una tendenziale predittibilità, prevedibilità e natura compromissoria.

La democrazia sarebbe il frutto dell'interdipendenza di interessi antagonistici e del tentato amalgama di ideali tra loro discordanti. Due regole sono intangibili: non può dichiararsi scacco matto ad uno dei giocatori [cioè: va rispettato il diritto alla proprietà privata della borghesia], né si può circoscrivere l'ambito di movimento della regina [cioè: non si possono minacciare le forze armate]. Corollari sarebbero: che ogni giocatore lotti per i propri pezzi e 
Di Palma $(1990)^{12}$ descrive la transizione come l'intaglio artigianale di una (nuova) democrazia. Il cesello riguarderebbe la forgiatura della comunità politica, delle convergenze tra forze politiche, dei meccanismi di negoziazione, degli istituti costituzionali stessi [«crafting institutions)].

Whitehead $(1997)^{13}$ si affida all'analogia con il teatro: la transizione condensa complesse, spesso imprevedibili narrazioni in gran numero in uno spazio cronologico limitato, per raggiungere un momento di risoluzione che si suppone cristallizzi il significato di quanto è venuto prima.

\subsection{Modi, tipi e processi di transizione}

Il "modo" ha riguardo, in senso lato, alla forma peculiare in cui la transizione si verifica. La nozione è capace di ricomprendere l'essere dei soggetti e il loro agire (ed in questa accezione si utilizza di solito il mode of transition), ${ }^{14}$ le cause, il contesto, la procedura.

Il "processo" è concetto che appartiene piuttosto alla scansione, se con esso si intende individuare la serie di fasi conseguenti che volgono verso il fine comune del cambio di regime.

Entrambi (modo e processo) possono assurgere a modello («tipo di transizione") che consenta di ricavare ordinamenti dai tratti comuni tra loro e quindi di operare per categorie.

luoghi, anziché per eliminare gli oppositori; e che i giocatori non necessariamente devono aver raggiunto un accordo previo sui valori democratici prima di cominciare la tenzone. Così O'Donnell G. e Schmitter P. C., "Definición de algunos conceptos (y exposición de algunas premisas)", in: O'Donnell G., Schmitter P. C., Whitehead L., Transiciones desde un gobierno autoritario, Tomo IV, op. cit., pp. 19-20 e 118.

12 Di Palma G., To Craft Democracy: An Essay on Democratic Transition, Berkeley, 1990.

13 Whitehead L., The Drama of Democratization, Juan March Institute, Madrid, Working Paper No 105, dicembre 1997.

Successivamente, in forma breve, in: Journal of Democracy, vol. 10.4, 1999, p. 84 ss.

$14 \mathrm{Il}$ «modo di transizione» è definito in termini di identità degli attori che guidano la transizione e di strategie da questi impiegate. Il concetto è funzionale a discernere i differenti processi per cui ci si disfa delle regole che definiscono i regimi politici. Cfr. Munck G. L. e Skalnik Leff C., "Models of Transition and Democratization. South America and Eastern Europe in comparative perspective», in: $C P$, vol 29, 1997, pp. 343-344. 


\subsection{Sequenza transizionale}

Sono stati esperiti numerosi tentativi per scandire una progressione «per tappe» della transizione.

In realtà ogni ricostruzione si rivela estremamente pericolosa perché estremamente artificiosa. Il caotico processo di cambiamento procede, arranca, arretra, devia e certo non in modo regolare. ${ }^{15}$ Tra il punto di partenza (il «significativo" sgretolamento del regime autocratico) e il punto d'arrivo si possono identificare con sufficiente approssimazione quattro fasi.

In limine: un grado "significativo" di erosione del governo autocratico si ha quando il regime, ancorché non esautorato [collapse], percepisce un calo tra i vantaggi di persistere ovvero tra $i$ costi per democratizzare ${ }^{16}$ e si inclina a modificare le proprie regole. ${ }^{17} \mathrm{Il}$ punto d'arrivo, per i più, è costituito dall'insediarsi di un governo elettivo ["instaurazione democratica"]; per Schmitter ${ }^{18}$ è la — successiva - ricognizione che non sussiste più alcuna minaccia di ristabilimento dello status quo ante, per Linz e Stepan il cambiamento è completo solo quando - ancor più in là nel tempo- «il governo eletto detiene de facto l'autorità di decidere nuove politiche e i poteri esecutivo, legislativo e giudiziario espressi dalla nuova democrazia non devono condividere de jure le proprie attribuzioni con altre istituzioni.» ${ }^{19}$

15 Carothers T., "The End of the Transition Paradigm", in: Journal of Democracy JoD, vol. 13.1, 2002, pp. 5-20.

16 Share D. e Mainwaring S., "Transitions through Transation: Democratization in Brazil and Spainm, in: Selcher W. (a cura di), Political Liberalization in Brazil: Dilemmas and Future Prospects, Boulder, 1986, pp. 175-215, già in: Transitions from Above: Democratization in Brazil and Spain, Kellogg Working Paper No 32, dicembre 1984.

17 Discorrendo in termini di tipicità, O'Donnell e Schmitter incappano nella confusione tra liberalizzazione e democratizzazione. Le regole «autoritarie" che verrebbero blandendosi sarebbero quelle attinenti ai diritti di gruppi e individui. Vedi Transiciones desde un gobierno autoritario, op. cit., p. 20.

18 Schmitter P. C., "The Consolidation of Political Democracies: Processes, Rhythms, Sequences and Types", in: Pridham G., Transitions to Democracy. Comparative Perspectives from Southern Europe, Latin America and Eastern Europe, Aldershot-Brookfield, 1995, pp. 535-569.

19 Linz J. J. e Stepan A., "Problems of Democratic Transition and Consolidation. Southern Europe, South America, and Post-Communist Europe», Baltimore-London, 1996, pp. 55-65 (trad. it Transizione e consolidamento democratico, Bologna, 2000, pp. 91-104). 
Le fasi si possono riassumere come:

1. liberalizzazione politica; ${ }^{20}$

2. attivazione di procedure minime di autogoverno. Le forze politiche vanno definendo la propria identità, i propri interessi, gli obiettivi e sviluppano credenziali di leadership;

3. riconoscimento e garanzia delle libertà pubbliche;

4. indizione e svolgimento di founding elections.

A loro volta, le quattro tappe si inscrivono nella dicotomia classica liberalismo-elettoralismo. È universalmente accettato soltanto come i due tratti debbano - prima o poi- rinvenirsi non alternativamente.

Esiste un concetto errabondo all'interno di una evoluzione così stilizzata. Il "punto di non ritorno" per chi scrive ${ }^{21}$ e per Di Palma ${ }^{22}$ si situa idealmente all'interno della scansione, in un momento non precisato né precisabile, in cui diviene fisiologico per i revanscisti opporsi alla democrazia a mezzo delle regole democratiche. Per Schmitter, come si è osservato, lo stesso si alloca dopo la trasferimento formale dei poteri, ed è un momento (quello estremo) della transizione che avviene in pieno consolidamento democratico.

La concezione "dinamica» del processo di transizione si deve a Rustow. ${ }^{23}$

Secondo l'Autore, vi sono alcuni «ingredienti» o passaggi indispensabili alla genesi di una democrazia: un sentimento di unità nazionale, come requisito-base (a), ed un percorso che si snoda attraverso la lotta [radicati e seri conflitti (b)], il compromesso [la cosciente adozione di regole democratiche (c)] e l'abitudine [di politici ed elettorato a tali regole (d)]. Tali elementi devono essere assemblati uno alla volta. Ogni compito ha la sua logica e i suoi protagonisti naturali.

20 Sia consentito il rinvio, sul punto, a Mezzetti L., Le democrazie incerte. Transizioni costituzionali e consolidamento della democrazia in Europa orientale, Africa, America Latina, Asia, Torino, 2000, pp. 191-202.

$21 \mathrm{Cfr}$. Le democrazie incerte. Transizioni costituzionali e consolidamento della democrazia in Europa orientale, Africa, America Latina, Asia, op. cit., p. 134 e 232.

22 Di Palma G., "Le transizioni democratiche in Europa Orientale. Una prospettiva comparata", in: Rivista Italiana di Scienza Politica, vol. 20, 1990, pp. 203-241.

23 Rustow D. A., "Transition to Democracy: Toward a Dynamic Model", in: $C P$, vol. 3, 1970, pp. 337-363. Il valore - ma anche il limite- dell'opera sta nell'essere stata elaborata prima della c.d. "terza ondata». 


\section{Classificazioni [«i tipi di transizione»]}

Per Laurence Whitehead è meritorio, quale sia l'esito sortito, il tentativo di dare ordine alla "confusa e contingente molteplicità di eventi e iniziative che paiono essere concatenate senza un'apparente struttura durante il compresso intervallo che va dalla disintegrazione di un sistema [Lato sensu] autoritario e l'emergere di un'alternativa democratica». Tanto a supplire ad errori e carenze, quanto a rivelare incoerenze o incongruenze, dovrebbe provvedere una strategia di analisi votata all'interpolazione dei modelli, favorita da un necessario pluralismo metodologico. ${ }^{24}$

Alfred Stepan (1986) ${ }^{25}$ si premura di avvertire che, come tutte le tipologie di scienze sociali, anche per gli idealtipi "transizione» trattasi di mera semplificazione di una realtà più complessa, che spesso coinvolge più tipi di percorso in concomitanza o in sequenza, laddove ogni singolo percorso è la risultante di una costellazione di variabili.

\subsection{Tipologia per strategia degli attori}

Juan J. Linz, Scott Mainwaring e Samuel P. Huntington hanno cercato di contornare idealtipi secondo il grado di controllo sul processo di transizione esercitato dall' élite governante uscente.

$\operatorname{Linz}^{26}$ traccia una dicotomia tra transizione mediante reforma del regime, cambiamento graduale (ad esempio Turchia, 1947) e negoziato (ad esempio Spagna, 1975-1978), e transizione mediante ruptura, cambiamento di natura rapida e radicale.

Nel primo caso, la trasformazione è condotta da chi detiene il potere (autocratico) in perfetta solitudine (Turchia, 1947) per scongiurare un destino che si profila avverso o nefasto, oppure, per essere il momento tardivo o le determinazioni esitanti, perché compromesse risultano le possibilità di un accordo con l'opposizione al regime, oppure, infine,

24 Whitehead L., The Drama of Democratization, Juan March Institute, Madrid, Working Paper No 105, dicembre, 1997.

25 Stepan A, "Paths toward Redemocratization: Theoretical and Comparative Considerations", in: O'Donnell G., Schmitter P. C. e Whitehead L., Transitions from Authoritarian Rules, vol. III, Baltimore, 1986, pp. 64-84.

26 Linz J. J., "Crisis, Breakdown, and Reequilibration", in: Linz J. J. e Stepan A., The Breakdown of Democratic Regimes, Baltimore, 1978, p. 35. 
perché il governo autoritario ritiene di aver compiuto la propria missione e quindi si dice disposto a ripristinare la democrazia, coerentemente con le premesse del proprio insediamento.

Più realisticamente la reforma è pactada, controllata dalla leadership riformista del regime uscente, ma portata avanti con la collaborazione, in forme svariate, dell'opposizione.

Nel secondo caso, la rottura, si attua un cambio del sistema istituzionale in cui non è la vecchia nomenklatura a dirigere; anzi, accade che la stessa si riscopra del tutto tagliata fuori, suo malgrado.

Lo stesso Linz definisce «un falso problema» l'alternativa tra riforma e rottura. ${ }^{27}$ Nella realtà dei fatti, nessuna delle due si concretizza in modo puro: la transizione è resa possibile dalla simultanea presenza di elementi di entrambe (traiettoria evolutiva di stampo transattivo: modello «reforma pactada-ruptura pactada»). Poiché sia "autocrati» che democratici dispongono di notevole forza o, viceversa, si presentano estremamente deboli, a causa dell'apatia di vasti segmenti della popolazione.

Mainwaring ${ }^{28}$ propone una tripartizione in transition after regime breakdown or collapse, in cui un rilevante cambiamento istituzionale $\mathrm{e}$ l'infrazione degli schemi autocratici avvengono senza che le élite "autoritarie" possano esercitarvi alcun controllo; transition through extrication, nella quale il regime non democratico, pur costretto a ritirarsi dal potere a causa del basso grado di legittimazione e di coesione interna, riesce ad imporre limiti alla forma e allo sviluppo cronologico del cambio politico; transition through transaction, in cui è per scelta del regime

27 Linz J. J., "Transitions to Democracy", in: The Washington Quaterly, vol. 13.3, 1990, pp. 143-164.

28 Viola E. e Mainwaring S., Transitions to Democracy: Brazil and Argentina in the 1980s, Kellogg Institute, Working Paper No 21, luglio, 1984, anche in: Journal of International Affairs, vol. 38, pp. 193-219. Cfr. altresì Share D. \& Mainwaring S., "Transitions through Transation: Democratization in Brazil and Spain", in: Selcher W. (a cura di), Political Liberalization in Brazil: Dilemmas and Future Prospects, Boulder, 1986, pp. 175-215, già Transitions from Above: Democratization in Brazil and Spain, Kellogg Institute, Working Paper No 32, dicembre 1984; Mainwaring S., "Transitions to Democracy and Democratic Consolidation: Theoretical and Comparative Issues", in: Mainwaring S., O'Donnell G. e Valenzuela A. (a cura di), Issues in Democratic Consolidations: The New South American Democracies in Comparative Perspective, Notre Dame, 1992, pp. 321-326.

E di Di Palma G. l'espressione «transition through transation». 
autocratico che prendono forma liberalizzazione e democratizzazione, ma questo, con lo scorrere del tempo, vede assottigliarsi vistosamente la propria influenza, pur rimanendo una forza significativa nel quadro istituzionale, capace di influenzare fortemente ma in modo circoscritto il corso del cambiamento politico.

L'Autore avverte come si tratti di idealtipi e come nessun caso reale si possa conformare completamente ad essi. Al contrario, ogni transizione alla democrazia è caratterizzata da negoziazione costante.

Huntington (1991) ${ }^{29}$ stilizza parimenti un modello tricotomico. Per aversi transformation è necessario che al governo i riformatori siano più forti dei conservatori intransigenti, che il governo sia più forte dell'opposizione e che all'interno di quest'ultima i moderati prevalgano sugli estremisti. Non è escluso che, con l'andar del tempo, gli oppositori moderati, comunque interpellati, siano cooptati nel governo, mentre si tende ad estromettere le ali reazionarie dello stesso. Nel replacement ha un ruolo di primazia l'opposizione, capace di imporsi mentre il regime, controllato dai duri, crolla. Il cammino che porta alla sostituzione inizia con una compatta lotta al governo non democratico, ma, successivamente al tracollo di questo, le opposizioni si frantumano in correnti che entrano in conflitto per il potere e dissentono quanto alla forma da attribuire al regime nascente. Librido del transplacement prevede che la transizione sia il frutto della collaborazione tra riformatori e oppositori moderati, capaci entrambi di controllare le fazioni antidemocratiche che si agitano negli schieramenti. Prerequisito al compromesso è il reciproco riconoscimento nell'avversario di una controparte legittima. Affinché la negoziazione appaia un rischio minore rispetto allo scontro è necessario poi che i gruppi dominanti di governo ed opposizione abbiano una forza equivalente e vi sia incertezza su chi prevarrà in fine. L'accordo porta a una cogestione del potere ovvero all'opportunità mutualmente garantita di competere per la sua conquista.

29 Huntington S. P., The Third Wave. Democratization in the Late Twentieth Century, Norman, 1993, trad. It. La terza ondata. I processi di democratizzazione alla fine del XX secolo, Bologna, 1995, capitolo III. 


\begin{tabular}{|l|l|l|l|l|}
\hline \multicolumn{1}{|c|}{$\begin{array}{c}\text { Controllo sulla } \\
\text { Transizione }\end{array}$} & \multicolumn{1}{|c|}{$\begin{array}{c}\text { Linz 1978 e } \\
1990\end{array}$} & \multicolumn{1}{|c|}{$\begin{array}{c}\text { Mainwaring } \\
1984\end{array}$} & $\begin{array}{c}\text { Huntington } \\
1991-93\end{array}$ & e.g. \\
\hline Regime autocratico I & nforma & $\begin{array}{l}\text { transition through } \\
\text { extrication }\end{array}$ & transplacement & $\begin{array}{l}\text { Bolivia, } \\
\text { Uruguay }\end{array}$ \\
\hline Bilaterale II & $\begin{array}{l}\text { neforma pactada- } \\
\text { nuptura pactada }\end{array}$ & $\begin{array}{l}\text { transition through } \\
\text { transaction }\end{array}$ & transformation & $\begin{array}{l}\text { Spagna, } \\
\text { Brasile }\end{array}$ \\
\hline Opposizione III & muptrma & $\begin{array}{l}\text { transition after } \\
\text { regime breakdowen or } \\
\text { cllapse }\end{array}$ & replacement & $\begin{array}{l}\text { Portogallo } \\
\text { Argentina }\end{array}$ \\
\hline
\end{tabular}

Nonostante le sfumature che rendono peculiare ciascuno dei tre sistemi, appaiono forti le analogie tra i tipi isolati, non potendosi sostenere, in ultima istanza, che la distinzione è meramente nominale.

\subsection{Tipologia per «modo - in senso stretto- di transizione»}

L'intuizione è di Terry Lynn Karl, ${ }^{30}$ ripresa da Philippe C. Schmitter ${ }^{31}$ e ottimizzata in due successive occasioni da Gerardo L. Munck. ${ }^{32}$

Il concetto di «modo di transizione» ${ }^{33}$ deve essere bidimensionale: nella fluidità del processo deve ritagliare — per definirla— l'identità di coloro che guidano il cambiamento [il «chi»] e i mezzi da questi adoperati [il

30 Karl T. L., "Dilemmas of Democratization in Latin America", in: AA. VV., América Latina en el Umbral de los años 90. [Colección de ponencias del Simposio Internacional, Beijing, 8-10 giugno 1988], Pechino, s.d., pp. 110-126, successivamente in: CP, vol. 23, 1990, pp. 1-21.

31 Karl T. L. \& Schmitter P. C., "Modes of Transition in Latin America, Southern and Eastern Europe», in: International Social Science Journal, vol. 128, 1991, pp. 269-284.

32 Munck G. L., Disaggregating Political Regime: Conceptual Issues in the Study of Democratization, Kellogg Institute, Working Paper No 228, New Haven, agosto 1996; Munck G. L. e Skalnik Leff C., "Models of Transition and Democratization. South America and Eastern Europe in comparative perspective», in: CP, vol 29, 1997, pp. 343-362.

33 Whitehead critica l'eccessivo volontarismo della democratizzazione come "patto tra elitem. In una simile ricostruzione rimane in ombra il tema del modellamento della comprensione e del sostegno popolare per gli accordi raggiunti.

Il voluntarism estremo suppone un'inesistente volontà dirigente unificata che controlli l'intero processo di cambio.

Vedi. Whitehead L., The Drama of Democratization, Juan March Institute, Madrid, Working Paper No 105, 1997. 
"come»]. Non è sufficiente considerare le strategie (come sub 2.1): occorre interpolarle con la variabile rappresentata dal soggetto portatore dell'impulso e sforzo sottesi al cambio.

Dall'intersezione tra mezzo principale [scontro versus compromesso] e attore principale [élite governante versus contro-élite] ${ }^{34}$ scaturiscono quattro idealtipi collocati agli estremi: cauta riforma, rivoluzione dall'alto/ imposizione, rivoluzione tout court, riforma tout court. In realtà, la combinazione è assai variegata, gli interstizi non classificabili.

Munck riesce a collocare nello schema embrionale di Karl le categorie elaborate dai politologi attenti prevalentemente alla strategia.

\begin{tabular}{|c|c|c|c|c|c|}
\hline \multirow[t]{2}{*}{ Strategia } & \multicolumn{5}{|c|}{ Identità dell' Agent of Change } \\
\hline & élite titolare & & combinazione attori & & contro-élite \\
\hline $\begin{array}{l}\text { unilaterale } \\
\text { [scontro] }\end{array}$ & $\begin{array}{l}\begin{array}{l}\text { revolution from } \\
\text { above }\end{array} \\
\end{array}$ & & $\begin{array}{l}\text { reform through } \\
\text { rupture III }\end{array}$ & & social revolution \\
\hline 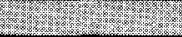 & Fin & & 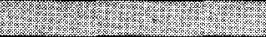 & 20 & 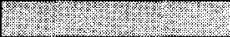 \\
\hline $\begin{array}{l}\text { combinazione } \\
\text { attitudini }\end{array}$ & & & $\begin{array}{l}\text { reform through } \\
\text { extrication I }\end{array}$ & & \\
\hline & 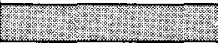 & & 1. & & 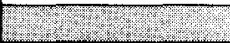 \\
\hline $\begin{array}{l}\text { multilaterale } \\
\text { [compromesso] }\end{array}$ & $\begin{array}{l}\text { conservative } \\
\text { reform }\end{array}$ & & $\begin{array}{l}\text { reform through } \\
\text { transation II }\end{array}$ & ?7? & reform from below \\
\hline
\end{tabular}

Risulta tuttavia poco convincente un'allocazione così "centrale» delle tre categorie classiche ${ }^{35}$ e diventa necessario modificare il reticolo e utilizzare categorie più univoche:

\begin{tabular}{|c|c|c|c|}
\hline Strategia & \multicolumn{3}{|c|}{ Identità dell' Agent of Change } \\
\hline & élite titolare & combinazione attori & contro-élite \\
\hline $\begin{array}{l}\text { unilaterale } \\
\text { [scontro] }\end{array}$ & $\begin{array}{l}\text { revolution from } \\
\text { above }\end{array}$ & & social revolution \\
\hline 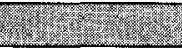 & & 3 & \\
\hline combinazion & & Ruptura pactada & \\
\hline attitudini & $\sqrt{28}$ & Reforma pactada & \\
\hline & & & 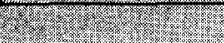 \\
\hline $\begin{array}{l}\text { multilaterale } \\
\text { [compromesso] }\end{array}$ & $\begin{array}{l}\text { conservative } \\
\text { reform }\end{array}$ & $\begin{array}{l}\text { reform through } \\
\text { transation }\end{array}$ & $\begin{array}{l}\text { reform from } \\
\text { below }\end{array}$ \\
\hline
\end{tabular}

34 In realtà, Karl tracciava un continuum tra élite e masse, non riuscendo perciò ad inquadrare "una questione cruciale nelle transizioni: lo scontro tra incumbent élite e 
I quattro idealtipi di transizione posizionati agli angoli lasciano consistenti (inter)spazi indeterminati. In questi territori virtualmente illimitati, interstizi tanto vasti da poter ricomprendere una infinità di ipotesi e da costituire un ostacolo alla cristallizzazione di modelli funzionanti, risiede la maggior parte delle transizioni che la realtà registra. Ciononostante, i $4[+3]$ idealtipi sono anche sistemi storicamente esistiti.

Il Cile rappresenta al meglio la reform from below: l'impeto al cambiamento veniva da gruppi che erano esclusi e fortemente avversati dal regime militare. Tuttavia, eludendo il regime change, l'élite al potere ha potuto esercitare un controllo sulla transizione al massimo grado, rispetto ad ogni altra ipotesi, tanto che gli oppositori si videro costretti a muoversi all'interno del quadro istituzionale del vecchio regime, mediante una strategia di mero accomodamento. La Bulgaria si colloca agli antipodi, con un prototipo di revolution from above. Eventi esterni hanno spezzato gli equilibri interni e preparato un'apertura preventiva dall'alto. Segmenti dell'élite al governo hanno avuto l'abilità di rompere col vecchio sistema e definire in solitudine un'agenda di transizione dall'alto. ${ }^{36} \mathrm{Il} \mathrm{Ni-}$ caragua incarna il formato della social revolution, per quanto sia discutibile che il processo intrapreso nel 1979 sia stato preordinato alla democrazia. Il puntofijismo venezuelano potrebbe concretizzare una conservative reform, ma sul punto occorre evitare di assestarsi ad una mera liberalizzazione,

counterélite». Cfr. Munck G. L. e Skalnik LeffC., «Models of Transition and Democratization. South America and Eastern Europe in comparative perspective", in: CP, vol 29, 1997, p. 361. E nel pensiero di Munck che il soggetto-chiave divengono le élite e non i gruppi d'interesse, le organizzazioni di massa, i movimenti sociali, in piena rispondenza da quanto preconizzato da Rustow nella decision phase. Cfr. Rustow D. A., Transitions to Democracy. Toward a Dynamic Model, op. cit., passim.

Per esse, "muoversi strategicamente" significa decriptare il comportamento di alleati e rivali, trarne delle aspettative, agire di conseguenza.

35 Desta perplessità, in particolar modo, l'extrication, il «trarsi d'impaccio», lo sbrogliarsi dagli attuali governanti, visto come terza via tra scontro e compromesso in un contesto in cui intervengono entrambi gli attori di vertice. La reforma può avvenire anche in perfetta solitudine (Linz) e lo stesso dicasi, in via tendenziale, per la transition after regime breakdown or collapse (Mainwaring).

In realtà, già nell'edizione del 1996 Munck aveva provveduto a lasciare spazio "al di sopra" della reform through rupture: Munck G. L., Disaggregating Political Regime: Conceptual Issues in the Study of Democratization, Kellogg Institute, Working Paper No 228, New Haven, agosto 1996 , passim.

36 Il partito comunista, non senza operazioni di restyling, ha ottenuto, a mezzo di vittoria elettorale, il potere di ritagliare le norme del nascente sistema. 
prestando attenzione al fatto che una democratizzazione realmente abbia preso piede. ${ }^{37}$

\subsection{Tipologia per capacità di ingenerare consenso}

In altra sede si è denunciata l'incompletezza ermeneutica del paradigma classico ${ }^{38} \mathrm{e}$ si è auspicata la adozione di un criterio ulteriore, integrante e perfettivo: «l'entità qualitativa e quantitativa del consenso che accompagna i processi [di democratizzazione]... nonché la identità dei soggetti protagonisti». ${ }^{39}$

Il "consenso" è parte del meccanismo di legittimazione politica: è il controcanto all'uso legale ed efficace del potere acquisito nel rispetto di un tessuto di norme generalmente condivise. ${ }^{40} S$ 'intende per "consenso" «il riconoscimento del diritto del governo ad emettere comandi e l'assunzione del dovere di obbedirvi» ${ }^{41} \mathrm{da}$ parte del singolo individuo in genere e della collettività nei regimi democratici in specie.

Trattando dell'ampiezza del consenso, in termini di pervasività, dunque, poichè un'analisi qualitativa si sottrae ad ogni schematismo, si potrebbe abbozzare una tavola, senza pretese di esaustività e con il solo fine di segnare i tipi di regime change per capacità, in via tendenziale, di ingenerare consenso.

\begin{tabular}{|c|c|c|c|}
\hline \multirow[t]{2}{*}{ Consenso } & \multicolumn{3}{|c|}{ Identità degli Attori } \\
\hline & tra élite & gruppi & masse \\
\hline ristretto/basso & managed transition ${ }^{1}$ & conditional transition & revolution \\
\hline medio-basso & transplacement & refolution & insurrection \\
\hline & & 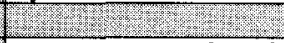 & 30 \\
\hline diffuso/alto & [élite-]pacted transition & $\begin{array}{l}\text { transition through } \\
\text { national conference }\end{array}$ & $\begin{array}{l}\text { government change via } \\
\text { democratic elections }\end{array}$ \\
\hline
\end{tabular}

37 Il rischio è, altrimenti, quello di porsi al di fuori del quadro transizionale. D'altro canto occorre evitare la teorizzazione di modelli vacui, incapaci di accogliere alcun caso concreto.

38 Si tratta della tripartizione dismissione volontaria del potere (I), transizione negoziata (II), surrogazione per implosione (III).

39 Cfr. Mezzetti L., Le Democrazie Incerte. Transizioni costituzionali e consolidamento della democrazia in Europa orientale, Africa, America Latina, Asia, op. cit., pp. 573-574.

40 In un'ottica soggettiva il consenso sta ai governati come il potere sta ai governanti. Elementi oggettivi che vanno a completare il quadro sono le regole di competizione, accesso ed esercizio del potere; elementi psicologici -nei governanti-sono il rispetto delle regole di accesso e quello delle regole di esercizio. 
A livello di élite, si può presumere che una transizione negoziata produca un grado medio-alto di consenso, salvo il caso in cui la stessa non sia guidata, dal basso e non sempre con mezzi democratici, magari da un unico leader (ad esempio Ghana, Kenya).

Suscita maggiore adesione la transizione i cui attori principali non si limitino agli elitisti.

Quanto ai gruppi, le conferenze nazionali (ad esempio Polonia, Germania orientale, Cecoslovacchia, ${ }^{43}$ Africa occidentale francofona, Sudafrica) sono un veicolo privilegiato e privilegiante, particolarmente efficacie in sede di liberalizzazione. La refolution ungherese ne costituisce una storpiatura, a causa del ruolo preminente svolto dai nuovi esponenti del partito fino ad allora sovrano e a causa del ritardo dei negoziati, fattori questi in grado di ridurre l'adesione collettiva, adesione del tutto inficiata dalle transizioni condizionate e sclerotizzate fino al blocco (ad esempio Algeria, Tunisia) a causa dell'azione stessa di taluni gruppi.

A livello di masse, una rivoluzione e, secondariamente, una generica insurrezione saranno capaci di attrarre minore consenso, specie in una prospettiva futura, rispetto ad un avvicendamento di regime implementato con mezzi democratici.

La tipologia illustrata, mentre si spinge a ragionare sul criterio da noi indicato, non può dirsi priva dell'ambiguità, dell'imprecisione e dei limiti che una prima schematizzazione porta connaturati.

\subsection{Tipologie ulteriori. Modelli parziali e modelli inconducenti}

Alfred Stepan ${ }^{44}$ identifica dieci "percorsi" rispetto alla ridemocratizzazione di un Paese. In linea di principio ripercorre l'antesignana dicotomia «transizione controllata dal regime -autocratico, in questo caso-

41 Mezzetti L., Le Democrazie Incerte. Transizioni costituzionali e consolidamento della democrazia in Europa orientale, Africa, America Latina, Asia, op. cit., p. 471.

42 Democracy through undemocratic means (O'Donnell).

43 Sulle «tavole rotonde» e le differenze tra ordinamenti dell'Europa centro-orientale sia consentito il riferimento a Mezzetti L., Le democrazie incerte. Transizioni costituzionali e consolidamento della democrazia in Europa orientale, Africa, America Latina, Asia, op. cit., pp. 47-51.

44 Stepan A, "Paths toward Redemocratization: Theoretical and Comparative Considerations", in: O'Donnell G., Schmitter P. C. e Whitehead L., Transitions from Authoritarian Rules, vol. III, Baltimore, 1986, pp. 64-84. 
uscente" (I) e "transizione controllata dall'opposizione» (III), ${ }^{45}$ matrice storica originata dall'analisi delle prime due transizioni di quella che si rivelerà poi essere la terza ondata di democratizzazioni (Portogallo, 1974 e Spagna, 1976). Alcune delle categorie indicate dall'Autore si rivelano essere null sets ${ }^{46}$ essenzialmente perché, nella realtà delle cose, la maggior parte delle transizioni ha carattere interattivo. Si ha per data, cioè, la coesistenza di risultanti di azioni concomitanti, pur se non coordinate, del regime uscente e dell'opposizione; sovente tale coesistenza approda fino ad un livello, per quanto embrionale, di negoziazione.

Donald Share ${ }^{47}$ elabora una matrice: in genere questo tipo di approccio (bidimensionale) è gravido di ottimi frutti, comunque dotati di maggiore complessità e, laddove non inutilmente capziosi, forieri di categorie che meglio rispondono a requisiti di opportunità, effettività e capienza.

\begin{tabular}{|l|l|l|}
\hline \multicolumn{1}{|c|}{ Andatura } & \multicolumn{2}{|c|}{ Consenso/Partecipazione dei Leaders Autocratici } \\
\hline & T. guidata dai Regime Leaders & T. guidata contro il regime \\
\hline graduale & $\begin{array}{l}\text { incremental consersual } \\
\text { democratization }\end{array}$ & transition through rewdutionary strugde \\
\hline rapida & $\begin{array}{l}\text { transition through transation II } \\
\text { transition through rupture III }\end{array}$ \\
\hline
\end{tabular}

45 Schema prodotto da Stepan:

I- Allorché il regime autoritario inizi e controlli il processo di democratizzazione [regime-led Transition]:

1 - ridemocratizzazione iniziata dai civili o dalla leadership civile; 2 - transizione iniziata dai "militari come governo"; 3- liberazione guidata dai «militari come istituzione»;

IIIa- Rispetto a guerra internazionale o intervento esterno:

4- ripristino interno dopo occupazione dall'esterno; 5- riformulazione democratica interna dopo liberazione dall'esterno; 6-installazione monitorata dall'esterno;

IIIb- Rispetto all'atteggiamento delle forze di opposizione:

7- la società nel suo complesso (guidata dalle forze d'opposizione) espelle il regime autoritario [society-led Transition]; 8- patto tra partiti politici di opposizione (al regime), con o senza elementi consociativi; 9-rivolta violenta; 10 -guerra di rivoluzione.

46 Precisamente, quelle qui indicate con i numeri $2,7,10$. Va notato poi che, ad aggravare la fragilità della ricostruzione, il tipo sub 9 ha registrato un unico caso storico (Costa Rica). Vedi Mainwaring S., Transitions to Democracy and Democratic Consolidation: Theoretical and Comparative Issues, op. cit., pp. 319-320.

47 Share D., Transition to Democracy and Transition through Transation, in Comparative Political Studies, vol. 19, 1987, pp. 525-548. 
Proprio quanto all'attitudine a ricomprendere casi attuali, le due categorie dell'andatura graduale si rivelano fallaci, ${ }^{48}$ rendendo quasi del tutto inutile il modello, ridotto a versione ridotta della tripartizione classica. Un'applicazione residua, ed estrema, potrebbe essere la seguente statuizione: "le transitions through reforma (I) non sono rapide». Il che, in linea di principio, non può che essere auspicabile, specie se il tipo si presenta come transplacement.

Al di là degli esperimenti di Stepan e di Share, appare chiaro come ogni modello sia destinato ad essere parziale finché non cercherà di temperare gli eccessi dello «strutturalismo» e del "transattivismo»: la transizione è il combinato disposto di negoziazione (e quindi, in ultima istanza, volontà) e coartazione (una sorta di determinismo storicocomplessionato).

Ciononostante, alcuni modelli si rivelano persino inconducenti, vacui epistemologicamente, falsamente ascrittivi.

Baloyra ${ }^{49}$ elabora una nebulosa tipologia quadripartita: early-internal, delayed-external, delayed-internal, late-external transitions. Ne è dubbia la conducenza e scarsa la capacità discretiva.

Morlino (1987) suggerisce un'ambiziosa analisi lungo la contestuale combinazione di nove variabili, analisi che originerebbe infinite «transizioni», forzosamente innominate. ${ }^{50}$

48 Non si conoscono casi di transition through revolutionary struggle, mentre il modulo della democratizzazione incrementale per negoziato controllato dal regime ha riguardato gli ordinamenti della prima ondata di democratizzazioni.

49 Baloyra, E., «Democratic Transition in Comparative Perspective», in: Baloyra, E., Comparing New Democracies: Transition and Consolidation in Mediterranean Europe and the Southern Cone, Boulder, 1987, pp. 9-52.

50 Morlino, L., “Democratic Establishments: A Dimensional Analysis», in: Baloyra, E., Comparing New Democracies: Transition and Consolidation in Mediterranean Europe and the Southern Cone, op. cit., pp. 53-78.

L'evanescenza della ricostruzione è denunciata da Mainwaring S., Transitions to Democracy and Democratic Consolidation: Theoretical and Comparative Issues, 1992, p. 319. Ricostruendo le variabili del modello come variabili bipolari, capaci di assumere due valori soltanto, si otterrebbero 512 (29) stringhe, vale a dire una quantità per cui un modello non è più tale. Tacendo del fatto che il numero degli Stati democratici si aggira attorno a 120, va evidenziato come difficilmente le variabili (si pensi al «livello di continuità nell'amministrazione») saranno suscettibili di concretarsi in due soli valori. Tendenzialmente si approda ad un prototipo alienante, dacché il numero delle "disposizioni con ripetizione» di 9 elementi di classe $¥$ è rappresentabile come $¥ 9$. 


\section{Paradigmi transizionali ulteriori}

Ogni analisi delle transizioni deve forzatamente fare i conti con la propria relatività.

Lungi dal costituirne un difetto, la ricognizione della intrinseca limitatezza di ciascun esperimento ricostruttivo è funzionale all'implicito accoglimento dell'integrazione operata mediante altre teorizzazioni e quindi, in ultima istanza, del pluralismo ricostruttivo. Linterazione di ogni paradigma emerge come necessaria e necessitata $a b$ origine; la cointeressenza appare percorribile nella misura in cui preclusioni non decurtino o spoglino un sistema per un altro, non assiomatizzino una logica per un'altra: in verità, povera sarebbe l'analisi che terminasse senza considerare l'innumerevolezza delle variabili registrabili a colorare una transizione. Data qualche costante, a caratterizzare le formule e gli schemi sono le variabili per lo più suscettibili di assumere infinite stringhe di valori, ricchezza in sé da non depauperare mediante moduli binari. In altri termini, i tratti fisionomici del cambiamento sono passibili di essere accorpati per campi semantici, a patto, tuttavia, di non snaturarli, di non eliderli per confusione, di non operare cernite o valorizzazioni gerarchiche abusive.

\subsection{Analisi per regime autocratico di partenza}

Juan J. Linz e Alfred Stepan elaborano una teoria della transizione incentrata sulla prospettiva dell' influenza ("eredità) esercitata dal precedente regime non democratico. ${ }^{51}$ Chiaro come neppure questo sia il solo fattore influente nella transizione e come la risultante sia una prospettiva

51 Linz J. J. \& Stepan A., Problems of Democratic Transition and Consolidation. Southern Europe, South America, and Post-Communist Europe, Baltimore-London, 1996, pp. 55-65 [cap. 4]; trad. It. parziale Transizione e consolidamento democratico, Il Mulino, Bologna, 2000, pp. 91-104.

In realtà, la costruzione elaborata dai due Autori riguarda le "transizioni" tout-court, intese nell'accezione ampia di "cambio di regime», piuttosto che in quella ristretta di "passaggio alla democrazia». I percorsi (paths) delineati si aprono ad una gamma di soluzioni: la democratizzazione, la pattuizione, l'indizione di elezioni, il governo provvisorio, un governo ancora non democratico, il caos. Nel totalitarismo [b] sconfitto rimane per natura sempre possibile lo sbocco del passaggio ad un post-totalitarismo $[c]$, non come percorso ma come risultato, mancano di precisare Linz e Stepan. 
tutt'altro che esaustiva, forse finanche troppo angusta, per ontologica limitatezza.

Il pregio della ricostruzione di Linz e Stepan risiede nel tentativo di elaborare un modello a due dimensioni sovraordinato alle costruzioni (anche bidimensionali) incentrate sui «modi di transizione». In altri termini, l'usuale tipologia, i modelli fin qui analizzati, anche quando derivanti da una griglia a doppia entrata, ${ }^{52}$ vengono comunque sussunti in un'unica variabile (per così dire "verticale»). Su questa, è implementata una elaborazione "orizzontale», frutto dello sforzo di Linz teso a isolare, all'interno del macrocosmo non democratico, sistemi politici distinti.

Per conferire sistematicità all'esposizione, si possono ricondurre le sette specie individuate dagli Autori allo schema monodimensionale classico dismissione volontaria del potere (I), transizione negoziata (II), surrogazione per implosione (III). Il che rappresenta, in definitiva, un passo indietro rispetto al modello di Karl e Munck. In altri termini: il disegno, nonostante l'auspicata tridimensionalità, manca di interpolare «regimi di partenza»e «modi di transizione», rimane bidimensionale, in quanto alla variabile «strategia degli attori» subentra la "tipologia di regimi non democratici", ferma restando la variabile «attori».

Ia- I «militari come istituzione», per varie ragioni, potrebbero fare pressioni sui «militari come governo» affinché le forze armate si ritirino dal governo diretto e si indicano elezioni libere. I militari verosimilmente potranno cadenzare il ritmo e la durata della transizione ed eventualmente imporre «domini riservati di potere». Il percorso è attuabile solo negli autoritarismi [a]..$^{53}$

Ib- Quanto ai regimi in condizione di dipendenza, nel totalitarismo [b], il ritiro dell'appoggio fondamentale di un soggetto egemone esterno può alterare tutti $\mathrm{i}$ rapporti di potere, aprendo la strada alla caduta del sistema. Il protettore straniero e il perdurare della crisi potrebbero indurre il sultano [d] a indurre elezioni in tempi brevi, nella presunzione di poterle

52 In verità, per ottenere un modello ricostruttivo che riesca a distinguere la percorribilità dei (vari) tracciati transizionali rispetto all'idealtipo di regime che ci si lascia alle spalle, si dovrebbe accedere ad uno spazio tridimensionale, estremamente complesso da concepire, in cui la tavola di Karl e Munck (dove le ascisse "x» sono l'identità dell'agente e le ordinate "y» le strategie) riceva una coordinata cartesiana ortogonale «z" (la quota dello spazio) in grado di rappresentare il sistema autocratico.

53 E' più facile, secondo Linz e Stepan, imporre un controllo da parte civile e iniziare anche procedimenti giudiziari se il regime uscente è diretto da militari non gerarchici. 
controllare. Non è esclusa la sconfitta elettorale per il sovrano o il suo abbandono, forzato dal protettore.

II- Una transizione pactada si rivela possibile, dati i tre prerequisiti indicati da Przeworski, ${ }^{54}$ solo in un regime (agonizzante) autoritario [a] o in uno post-totalitario (solo se maturo) [c].

IIIa. La sconfitta militare può condurre ad una transizione quando il regime autoritario [a] conservi una pur debole capacità negoziale a fronte di una società civile e politica che, disponibile, richieda un percorso elettorale. Il post-totalitarismo maturo [c] debellato rende percorribile l'assunzione del governo da parte di un'opposizione democratica e l'immediato svolgimento di elezioni. La sconfitta bellica deve rivestire la forma di occupazione da parte di un Paese democratico ed esser accompagnata da un'instaurazione democratica controllata dall'esterno, per poter uscire da un regime totalitario [b], post-totalitario ancora acerbo [c], o sultanistico [d].

IIIb. Nell'ipotesi di caduta del regime non democratico, ossia di cambio non avviato dallo stesso regime — si spazia dal colpo di stato, alle rivolte armate, alle insurrezioni di massa-, può darsi l'insediamento di un governo interino con il compito di portare il Paese al voto. Quando a cadere siano autoritarismi [a] o post-totalitarismi maturi [c], può darsi che l'opposizione sia sufficientemente organizzata da richiedere con successo elezioni. Altrimenti -l'affermazione è valida in particolare per il crollo del regime sultanistico [d] ${ }^{55}$ - un governo ad interim potrebbe esser tentato di esercitare poteri rivoluzionari in vari settori della vita politica,

54 Przeworski ricava dalla teoria dei giochi un modello con 4 attori. In esso: a- i moderati del regime e i moderati dell'opposizione debbono esser entrambi in grado di usare e controllare i propri rispettivi estremisti;

b- i moderati del regime devono esser dotati di sufficiente autorità per poter condurre negoziazioni (sia strategiche che tattiche) con i moderati dell'opposizione;

c- $\mathrm{i}$ moderati dell'opposizione necessitano di un certo grado di presenza organizzativa continuativa, di potere e di sostenitori all'interno del sistema politico (cd. "opposizione democratica organizzata non-violenta).

Vedi «The Games of Transition», in: Mainwaring S., O'Donnell G. \& Valenzuela S. (a cura di), Issues in Democratic Consolidation, Notre Dame, 1992, pp. 105-153.

55 In buona misura, la causa interna più comune della rimozione del sultano è l'assassinio o lo spodestamento per sollevamento rivoluzionario (specie da parte di gruppi armati) ed è molto probabile, in queste ipotesi, che si instauri un governo provvisorio. Se, per contro, il sultano muore per cause naturali, i membri della famiglia cercheranno di perperuare il regime. 
dilazionando il termine per le elezioni. Un governo ad interim è improbabile dopo un regime totalitario $[b]^{56} \mathrm{e}$, al più, cercherebbe elezioni per legittimarsi. Il post-totalitarismo precoce e congelato [c] o il sultanismo in genere [d] saranno sostituiti da un governo in buona misura equivoco in caso di insurrezione di massa che abbia successo.

\subsection{Analisi per condizione del sistema economico di partenza}

Stephan Haggard e Robert R. Kaufman ${ }^{57}$ si staccano dalle analisi «in soggettiva", per distinguere le transizioni che hanno luogo in una congiuntura di crisi economica da quelle che godono di un momento di buona performance economica del Paese.

Nel prospetto a due tipi, gli Autori indicano alcuni temi lungo i quali si è storicamente ripartito uno stock di dieci casi empirici. ${ }^{58}$

Non può sottovalutarsi a priori il procedimento induttivo, soprattutto se funzionale all'elaborazione di nuovi paradigmi di analisi. Tuttavia, la tavola di seguito riportata arrischia un livello di astrazione ulteriore rispetto alle conclusioni offerte da Haggard e Kaufman.

\begin{tabular}{|l|l|l|}
\hline \multicolumn{2}{|c|}{ Questione Politica } & \multicolumn{2}{|c|}{ Situazione Economica } \\
\hline Sfida politica & $\begin{array}{l}\text { Le istanze politiche concorrono } \\
\text { con istanze economiche }\end{array}$ & $\begin{array}{l}\text { L'istanza è per la } \\
\text { liberalizzazione }\end{array}$ \\
\hline $\begin{array}{l}\text { Riforma } \\
\text { costituzionale }\end{array}$ & $\begin{array}{l}\text { Forte influenza } \\
\text { dell'Opposizione }\end{array}$ & Dominio degli autocratici \\
\hline $\begin{array}{l}\text { Pubblici ufficiali e } \\
\text { assetto istituzionale }\end{array}$ & $\begin{array}{l}\text { Giurisdizione degli ufficiali } \\
\text { elettivi }\end{array}$ & $\begin{array}{l}\text { Manca una netta troncatura con } \\
\text { glistituti dell'ancient regime }\end{array}$ \\
\hline $\begin{array}{l}\text { Ostacoli all'accesso } \\
\text { politico }\end{array}$ & $\begin{array}{l}\text { Partecipazione tendenzialmente } \\
\text { libera e leggi elettorali } \\
\text { includenti }\end{array}$ & $\begin{array}{l}\text { Legislazione elettorale } \\
\text { escludente; limitazioni a } \\
\text { determinati gruppi politici }\end{array}$ \\
\hline Party Arena & $\begin{array}{l}\text { Sistema partitico frammentato } \\
\text { e/o polarizzato }\end{array}$ & $\begin{array}{l}\text { Forti partiti di continuita in un } \\
\text { Sistema centristico }\end{array}$ \\
\hline
\end{tabular}

56 Ma potrebbe dividersi la leadership, aprendo lo spazio alla mobilitazione popolare la quale, a sua volta, può condurre ad un governo liberalizzatore interinale.

57 Haggard S. \& Kaufman R. R., "The Political Economy of Democratic Transitions", in: CP, vol. 29, 1997, pp. 263-283.

58 Quali Crisis Transitions sono considerate: Argentina (1983), Bolivia (1980), Brasile (1985), Filippine (1986), Perú (1980) e Uruguay (1985). Prototipi di Non-Crisis Transitions sono stati: Cile (1989), Corea del Sud (1986), Thailandia (1983) e Turchia (1983). 
Lungi dal dichiararsi in grado di concepire una "teoria economica della transizione democratica», Haggard e Kaufman non rigettano tuttavia l'affermazione per cui gli interessi e le relazioni sociali determinerebbero le aspettative per la democrazia. La possibilità per le élite politiche di mobilitare sostegno od opposizione nelle nuove democrazie dipenderà ancora dalle politiche e performances economiche. Per le Non-Crisis Transitions va notato come, pur rivelandosi meno pressanti le esigenze di riforme macroeconomiche, anche il miglior "autoritarismo» in economia lascia problemi di distribuzione, povertà, deficit sociale ed esclusione politica, passibili tutti di rivelarsi esplosivi in un contesto democratico.

\subsection{Analisi del modello socio-economico in tempo di transizione}

José Nun e Juan Carlos Portantiero ${ }^{61}$ sperimentano un difficoltoso ${ }^{62}$ inquadramento della transizione nello schema del conflitto tra [appropriazione e] accumulazione del "plus-valore» e distribuzione della ricchezza. ${ }^{63}$

È concezione rapsodica, che combina gli sbalzi del sistema politico con gli sbalzi del sistema sociale: talora i due fattori vengono a sovrapporsi, producendo una transizione duplice e complessa.

Prima facie si potrebbe obiettare che una simile costruzione inserisce elementi spuri nel tessuto del "cambio di regime». Il punto è che, per Nun, «regime» non è solo quello «politico». Esistono transizioni che si allocano all'interno di una crisi che è duplice: non solo crisi del «regime politico di governo", ma anche crisi del "regime sociale di accumulazione». ${ }^{64}$

59 Esemplificando, le frizioni si concretizzano in defezioni nella business élite, proteste di massa a fini economici e spaccature nel governo sul problema della distribuzione.

60 In tal senso si registrano sostanziali prerogative militari e la presenza di significative enclavi autoritarie.

61 Nun, J., La teoría política y la transición democrática; Portantiero J. C., "La crisis de un régimen: una mirada retrospectiva", entrambi in: Nun J. \& Portantiero J. C. (a cura di), Ensayos sobre la transición democrática en la Argentina, Buenos Aires, 1987.

62 Il pericolo è quello di confondere -come di fatto accade per Nun- la transizione e il consolidamento.

63 Già la tradizione sociologica e marxista evidenziava la distonia nell'allocazione disarmonica dei beni.

64 In altri termini, la crisi di una tappa capitalistica e delle strutture, istituzioni, immagini e tipo di attori che le sono propri. Cfr. Nun, J., La teoría politica y la transición democrática, op. cit., p. 48. 
Il "regime sociale di accumulazione» non coincide con il processo sociale di accumulazione capitalista (di concezione marxista), né corrisponde alla nozione classica (di Smith e Ricardo) di modo di produzione determinato dall'economia. Allude, piuttosto, all'insieme complesso delle istituzioni e delle pratiche che incidono sul processo di accumulazione del capitale, intendendo quest'ultimo come l'attività microeconomica di generazione di profitto e di assunzione di decisioni di investimento. Tale attività non può portarsi a capo né nel vuoto né nel mezzo di un caos. Per questo esiste un regime [sociale di accumulazione], accanto al «regime politico di governo".

Un «regime sociale di accumulazione» è insanabilmente eterogeneo ed è percorso da contraddizioni permanenti che si manifestano a livelli variabili di conflittività. $\grave{E}$ una matrice dalla cangiante configurabilità, al cui interno si vanno allacciando differenti strategie di accumulazione e tattiche per implementarle, di modo che "l'accumulazione di capitale appare sempre come il risultato contingente di una dialettica di strutture e strategie».

La transizione non può limitarsi a [ri-]stabilire le consultazioni elettorali e il libero gioco dei partiti politici. Necessita della costruzione di un terreno in cui si sviluppino e si integrino tre aspetti: il democraticorappresentativo, quello corporativo e quello capitalista. Altrimenti il primo aspetto (la democrazia rappresentativa) si configura come una «finzione organizzativa" che difficilmente funzionerà e men che meno si manterrà.

Lalternativa per la comunità si prospetta in questi termini: costruire un ordine basato sulla legge o abbandonarsi ad un processo di dissoluzione progressiva. La democrazia non si profila come la panacea, ma come uno strumentario per perseguire la giustizia sociale, a mezzo della regolazione dei conflitti e dell'adozione di decisioni collettive.

Il tentativo dei Paesi sottosviluppati è il paradosso di cercare la democrazia [rappresentativa] ${ }^{65}$ in contesti marcati da povertà, diseguaglianza e polarizzazione nella distribuzione del reddito e in cui i regimi sociali di accumulazione vigenti fomentano la marginalità $e$ l'esclusione. ${ }^{66}$

65 «In America Latina, la democrazia rappresentativa risulta perseguibile solo dentro gli stretti limiti che i politici devono continuamente negoziare con i grandi gruppi economici stranieri». Cfr. Nun, J., Democracia. ¿Gobierno del pueblo o gobierno de los políticos?, Buenos Aires, 2000, capp. XVIII e XXI.

66 Il capitalismo genera per se diseguaglianze, che nel caso delle società latinoamericane sono implementate su una eterogeneità strutturale, tratteggiata dalle diseguaglianze proprie 


\begin{tabular}{|l|l|l|}
\hline \multicolumn{1}{|c|}{$\begin{array}{c}\text { Fattori di } \\
\text { Democratizzazione }\end{array}$} & \multicolumn{2}{|c|}{ Contesto } \\
\hline & Paesi ricchi & Paesi Latinoamericani \\
\hline $\begin{array}{l}\text { Primo fattore: } \\
\text { di possibilità }\end{array}$ & $\begin{array}{l}\text { l'efficace protezione sociale, che } \\
\text { ha portato il Paese all'economia } \\
\text { di mercato }\end{array}$ & $\begin{array}{l}\text { gli Stati si rimpiccioliscono e si } \\
\text { rivelano incapaci di fronteggiare la } \\
\text { crisi }\end{array}$ \\
\hline $\begin{array}{l}\text { Secondo fattore: } \\
\text { di desiderabilità }\end{array}$ & $\begin{array}{l}\text { lidea che il potere legittimo } \\
\text { dello Stato si fondi sulla libera } \\
\text { elezione periodica }\end{array}$ & $\begin{array}{l}\text { la rappresentatività opera } \\
\text { prescindendo dalla protezione } \\
\text { sociale; il Sistema si fonda } \\
\text { sull'imitazione dei privilegi }\end{array}$ \\
\hline $\begin{array}{l}\text { Terzo fattore: } \\
\text { di sostenibilità }\end{array}$ & $\begin{array}{l}\text { un aumento generalizzato del } \\
\text { benessere (della popolazione) }\end{array}$ & $\begin{array}{l}\text { i regimi sociali di accumulazione } \\
\text { vigenti fomentano la marginalità e } \\
\text { l'esclusione }\end{array}$ \\
\hline
\end{tabular}

Una critica larvata all'approccio socio-economico viene da Whitehead. ${ }^{68}$

Se l'opzione democratica durante la transizione "riflette una spaccatura nel bilancio di lungo periodo del potere di classe, in che modo questo cambiamento nelle condizioni di base si converte nelle strategie e interazioni di decision-makers più pienamente individualizzati? Le azioni di tali politici possono esser lette alla luce del loro background di classe o non dovremmo anche considerare le loro convinzioni e motivazioni più personali?»

\subsection{Analisi del diritto della transizione}

Una prospettiva finora non adeguatamente considerata è quella dell'analisi dell'ordinamento giuridico nel suo complesso nell'intervallo della transizione.

In realtà, la transizione è anche un momento di intensa normazione. Il successo del cambio risiede in buona parte nella capacità che gli attori coinvolti devono dimostrare nel tratteggiare le regole, spesso non scritte, della negoziazione e dell'istituzionalizzazione, di "codificare" e, ove

di strutture di dominazione e sfruttamento segnatamente meno moderne di quelle dei Paesi sviluppati.

67 Nei Paesi ricchi, la democrazia è frutto di tre fattori, i primi due mutuati da John Dunn. Vedi Dunn J., "Conclusion", in: Dunn J. (a cura di), Democracy, New York, 1993.

68 Non si può pensare di trasferire de plano il modello dell'uomo economico nell'elaborazione classica e neoclassica-dalla sua particolare posizione sociale ad ogni altro dominio. Whitehead L., The Drama of Democratization, Juan March Institute, Madrid, Working Paper No 105, dicembre 1997. 
possibile, formalizzare pro futuro l'enteléchia e il principio vitale: la democrazia. Ma deve parlarsi di una minuta, pulviscolare attività lato sensu legislativa, ad opera del governo autocratico uscente o di un governo interino, a metà strada tra l'ancient regime e la futura articolazione democratica del Paese. E' intuitivo come, nella pur limitata letteratura strettamente giuridica, grande attenzione sia stata prestata al diritto costituzionale. ${ }^{69}$ Del resto, a ragione, si parla di «transizione costituzionali»" ${ }^{70}$ e di «ingegneria costituzionale». ${ }^{71}$

Linz e Stepan abbozzano una classificazione, indicando sei contesti in cui le formule costituzionali sono adottate o mantenute. ${ }^{72}$ Lesposizione avviene in ordine decrescente quanto alla capacità di intralciare la transizione [e il consolidamento].

1. Mantenimento di una Costituzione creata da un regime non democratico con «domini riservati» e difficili procedure di emendamento. Si chiuderà la transizione solo quando tali enclavi saranno rimosse (es. Cile);

2. Mantenimento di una Costituzione non democratica scritta, con manierate regole decisionali, procedure e diritti che nel periodo non democratico non hanno avuto alcun effetto perché la Carta era virtuale. Una volta applicata a un sistema elettorale più competitivo può avere inattese conseguenze destabilizzanti e paralizzanti. (es. ex-Urss, exIugoslavia);

3. Creazione di una Costituzione da parte di un governo provvisorio de jure ma non democratico. Ancora la transizione non può dirsi completa fintantoché non vengano eliminate le clausole non democratiche. $\mathrm{Ma}$ anche una volta rimosse queste ultime, il consolidamento sarà messo a repentaglio dalla inadeguatezza o debole accettazione sociale. (es. Portogallo);

69 In particolare: Elster J. \& Slagstad R. (a cura di), Constitutionalism and Democracy, Cambridge, 1988; Greenberg D., Katz S. N., Oliveira M. B., Wheatly S. C. (a cura di), Constitutionalism and Democracy: Transitions in the Contemporary World, New York, 1993.

70 Mezzetti L., Le Democrazie Incerte. Transizioni costituzionali e consolidamento della democrazia in Europa orientale, Africa, America Latina, Asia, op. cit., p. 571.

$71 \mathrm{Cfr}$., per tutti, Sartori G., Ingegneria costituzionale comparata, $4^{\circ} \mathrm{ed}$., Il Mulino, Bologna, 2000.

72 "A neglected aspect of democratic transition and consolidation", in: Linz J. J. \& Stepan A., Problems of Democratic Transition and Consolidation. Southern Europe, South America, and Post-Communist Europe, op. cit., p. 81. 
4. Attivazione del potere costituente, estremamente vincolato, però, da condizioni che riflettono il potere de facto di istituzioni e forze non democratiche. La Carta sarà formalmente democratica e quindi coerente con il completamento della transizione, ma ostacolerà il consolidamento (es. Brasile);

5. Restaurazione della precedente Costituzione democratica. Si ricorre a tale soluzione per evitare un dibattito foriero di spaccature, per ragioni di rapidità e per il desiderio di richiamarsi a una qualche eredità di legittimità storica ${ }^{73}$ (es. Argentina, Uruguay);

6. Libera e consensuale adozione di una nuova Costituzione, indifferibile allorché una Costituzione previgente manchi o si riveli inadeguata. L'Assemblea Costituente eviterà in linea di principio l'approvazione di una Carta di parte, approvata solo da una maggioranza temporanea, che porterebbe un'ampia minoranza a mettere in agenda revisioni costituzionali, rendendo allora tortuoso il consolidamento. Maggiore legittimità acquista l'opera approvata da un referendum popolare. (es. unico la Spagna).

Un'attenzione maggiore all'ordinamento giuridico nel suo complesso viene prestata dalla letteratura ispanofona. Enrique Groisman ${ }^{74}$ guida una linea dottrinaria che intende il diritto come un fenomeno storico riferito a (e spiegabile da) situazioni sociali concrete, in grado di ricevere quanto di esercitare influenze.

Il diritto in genere compie un ruolo positivo nelle battute iniziali della transizione, attraverso molteplici meccanismi di rivitalizzazione di norme, istituzioni e pratiche, contribuendo alla produzione di sentimenti funzionali alla legittimazione del nuovo ordine. Nella ricostruzione di Alicia Ruiz e Carlos María Cárcova, ${ }^{75}$ la legalità consente la produzione

73 Una condizione ed un limite: il ripristino presuppone che le procedure ele istituzioni della vecchia Costituzione non abbiano giocato alcun ruolo nel [precedente] crollo della democrazia; se il sistema politico, poi, ha conosciuto grandi cambiamenti durante l'interludio "autoritario", ai fini del consolidamento è più adeguata una nuova carta.

74 Groisman E. I. (a cura di), El Derecho en la transición de la dictatura a la democracia: la experiencia en América Latina, (2 voll.), Biblioteca politica argentina, No 300 e No 301, Buenos Aires, 1990.

75 Ruiz A. E. C. \& Cárcova C. M., «Derecho y transición democratica», in: Groisman E. I. (a cura di), El Derecho en la transición de la dictatura a la democracia: La experiencia en América Latina, vol. 1, Biblioteca politica argentina, No 300, op. cit., pp. 97-117. 
legislativa, la conoscenza giuridica e l'applicazione e interpretazione del diritto. «Il diritto è una domanda ineludibile del fondamento razionale dell'organizzazione democratica, poiché presuppone una coesistenza sociale basata sull'accordo libero e volontario di soggetti eguali». Carlos Santiago Nino scriveva, replicante ed icastico: «L'origine democratica di una norma ci offre ragioni per credere che ci sono ragioni per realizzare il contenuto di una norma. Nell'offrirci tali ragioni risiede la superiorità morale della democrazia, giacché abbiamo ragioni per credere che abbiamo ragioni per farem. ${ }^{76}$

Nel concreto, gli autocratici "agli sgoccioli» produrranno norme di apertura, volte a riservarsi quanto più possibile benefici e, al contempo, ad ingraziarsi la comunità. Mentre il governo democratico neoeletto si troverà ad affrontare il più spinoso dei problemi: ciò che tanto spazio occupa nel dibattito giuridico nei regimi post-autoritari è la questione del trattamento del diritto di fatto. Si tratta di decidere se invalidarlo in blocco o in parte, se smussarne le asperità e sopprimerne quanto di intollerabile, ovvero se recepirlo, integrandolo nell'ordinamento nascente. Il diritto dell'inaugurazione democratica avrà un occhio di riguardo per i diritti umani, dovrà farsi carico del [completamento dello] smantellamento del passato regime e della costruzione del nuovo.

Non è escluso che dall'analisi comparatistica emergano prototipi in grado di organizzare una nuova tipologia di transizioni. ${ }^{77}$

\section{Il futuro della Transitology}

L'esperienza e la necessità stessa di una nuova scienza, Transitology, così vivida per un decennio -indicativamente dal 1986 al 1995- soffre attualmente un momento d'incuria ed abbandono.

Alcuni segni, più o meno espliciti, della breve stagione sono: 1) la ricognizione del fallimento empirico di un intreccio di statuizioni fondanti il paradigma transizionale (Carothers), ${ }^{78}$ 2) l'individuazione di una gray

76 Nino, C. S., "El valor epistemológico de la democracia», mimeografia cit. da Ruiz \& Cárcova.

77 Sulle dimensioni di un'elaborazione teorico-empirica, vedi Munck G. L., “Democratic Transitions in Comparative Perspectiven, in: CP, vol. 26, 1994, p. 369.

78 «ll paradigma transizionale è stato il prodotto di un determinato tempo e quel tempo ora è passato": Carothers T., "The End of the Transition Paradigm", in: JoD, vol. 13.1, 2002, p. 19. 
$z o n e$ in cui giacciono in quantità gli ordinamenti neonati; l'interesse degli studiosi non più rinnovato. La letteratura sulla transizione è frorita, come è ovvio, specialmente nei dintorni dello tsunami «1989». La «terza ondata di democratizzazioni" si è andata successivamente affievolendo, forse progressivamente spegnendo e l'attenzione degli studiosi si è rivolta altrove ${ }^{79}$ in particolare al consolidamento democratico; 3) l'elaborazione di approcci radicalmente alternativi alla transizione, come la Developmental Democracy di Richard L. Sklar. ${ }^{80}$ Sklar ritiene lo sviluppo democratico un processo continuo e non la traversata della soglia di un tipo di regime. ${ }^{81}$ L'«evoluzione» risiede nella natura incessante ed aperta del cambiamento della democrazia e delle sue istituzioni.

\subsection{La fine del paradigma delle transizioni}

In un discorso rivolto ai democracy promoters, Thomas Carothers ${ }^{82}$ indica cinque assiomi, costituenti il nucleo della "teoria della transizione» che, a suo avviso, all'inizio del XXI secolo non sarebbero più validi in quanto smentiti da una ricognizione empirica delle traiettorie politiche dei «Paesi della terza ondata».

Le presunzioni incriminate sono:

1. "Ogni Paese che si distacchi da un regime dittatoriale può essere considerato un Paese in transizione verso la democrazia».

2. «La democratizzazione tende a spiegarsi in un insieme sequenziale di fasi».

79 Sintomatico come l'ultimo Working Paper del «Kellogg Institute for International Studies» dell'Università di Notre Dame intitolato alla transizione risalga al dicembre 1992. Vedi. Bartell E., Business Perceptions and the Transition to Democracy in Chile, Kellogg Institute, Working Paper No 184.

80 Sklar, R. L., "Towards a Theory of Developmental Democracy", in: Leftwich, A. (a cura di), Democracy and Development: Theory and Practice, Cambridge, 1996, pp. 25-44; Idem, Developmental Democracy, in Comparative Studies in Society and History, vol. 29.4, 1987, pp. 686-714. L'Autore si poggia sulle deficienze democratiche delle forme di governo costituzionali stabili e industrializzate e sui frammenti democratici presenti in varie forme di governo autocratico per rifiutare un «sistema" globale.

81 Per un confronto con la "scuola della transizione» vedi Diamond L., "El final de la tercera ola y el futuro global de la Democracia", in: López E. \& Mainwaring S. (a cura di), Democracia: discusiones y nuevas aproximaciones, Buenos Aires, 2000, pp. 39 e 45.

82 Carothers, T., "The End of the Transition Paradigm», in: JoD, vol. 13.1, 2002, pp. $5-20$. 
3. "Le elezioni hanno rilevanza determinante». Lo svolgimento di genuine elections implicherebbe per se ottimizzazione della partecipazione politica e rafforzamento della responsabilità del governo.

4. «Le pre-condizioni strutturali ${ }^{83}$ non sono fattori determinanti per l'inizio della transizione, né per il suo risultato".

5. «Le transizioni democratiche registratesi nella terza ondata sono state implementate su Stati già funzionanti e coerenti».

\begin{tabular}{|c|c|}
\hline The Transition Paradigm & smentita empirica \\
\hline 1. presunzione democratica & $\begin{array}{l}\text { Cambogia, Congo-Kinshasa, Guinea, Moldova, } \\
\text { Zambia }\end{array}$ \\
\hline 2. sviluppo sequenziale & Corea del Sud, Messico, Taiwan \\
\hline 3. centralità delle elezioni & Nepal \\
\hline 4. «No preconditions» view & $\begin{array}{l}\text { Africa sub-sahariana, Cono Sud delle Americhe, Eu- } \\
\text { ropa centrale, Estremo Oriente }\end{array}$ \\
\hline 5a. esistenza dello Stato & $e x$-Yugoslavia, ex-Urss \\
\hline 5b. efficienza dello Stato & America Latina, Asia (ptc Medio Oriente) \\
\hline
\end{tabular}

Ricavata la precedente tabella dall'opera di Carothers, si potrebbe essere tentati di sostenere che l'Autore ha esposto a titolo di esempio tutte le parti del mondo in disfunzione. Potrebbe, ciò̀, aver operato induttivamente. I postulati teorici potrebbero essere semplici cristallizzazioni di una somma di situazioni rilevate.

In realtà Carothers offre la concettualizzazione di uno spazio in cui sarebbero rifluiti tutti gli Stati per i quali la transizione pare non essere più un costrutto calzante.

\subsection{Un tertium genus}

Quanto spesso si è ritenuto una condizione precaria tra full-fledged democracy e outright dictatorship è attualmente lo status politico più comune nel mondo in via di sviluppo e in quello post-comunista. Non è categoria residuale, definita in termini negativi: è la condizione di normalità per

83 Esemplificando: il livello di sviluppo economico, la storia politica, le eredità istituzionali, la composizione sociale, la composizione etnica, le tradizioni socio-culturali. 
molte società. La maggior parte dei transitional countries non si rivela né autocratica (non più tale e non regredita) né democratica: tali ordinamenti, figli della terza ondata, non hanno raggiunto una forma democratica funzionante o non sembrano in grado di avanzare nel progresso democratico che pure ha attecchito al loro interno. ${ }^{84}$ Sono entrati in una "zona grigia». ${ }^{85}$

Questa depigmentazione spiegherebbe il proliferare della «qualified democracyn.

Ciascun "Paese della zona grigia» ha il proprio tratto dominante, pur essendo i più frequenti gracile pluralismo e dominio politico da parte di un gruppo che esclude l'alternanza al potere.

\section{Parte Seconda. I Processi Di Consolidamento Democratico}

\section{Il consolidamento della democrazia}

"Consolidamento" è un'etichetta imprecisa e generica che si piega a far rientrare nel processo di democratizzazione una congerie indefinita di elementi disparati e talora sconnessi rispetto al problema in senso stretto.

Si parla di consolidamento con riguardo alla legittimazione, alla diffusione di valori democratici, alla neutralizzazione di attori antisistema, alla supremazia dei civili sui militari, all'eliminazione di enclavi autoritarie, alla costruzione di partiti, all'organizzazione degli interessi, alla stabilizzazione delle regole elettorali, alla routinizzazione delle politiche, al decentramento, all'introduzione di meccanismi di democrazia diretta, alle riforme -in particolare giudiziarie-, all'alleviare la povertà, alla stabilizzazione economica. La tematica sembra non avere fine.

La vastità dei contenuti riconfluiti causa (ed è causata dalla) la non precisabilità dei confini del concetto. In tale contesto la letteratura usa, talvolta con leggerezza, termini dal distinto significato come fossero sinonimi: istituzionalizzazione, routinizzazione, stabilizzazione-stabilità, sostenibilità, radicamento, persistenza, approfondimento, perfezionamento, durevolezza, continuità, mantenimento, sopravvivenza, permanenza, resistenza, persistenza, resilenza, percorribilità, irreversibilità. Ed in senso negativo, il tracciato è nel senso di andar oltre la fragilità,

84 Carothers, T., "The End of the Transition Paradigm", in: JoD, vol. 13.1, 2002, pp. 17 e 9 . 
l'instabilità, l'incertezza, la vulnerabilità, la reversibilità, il timore di crollo.

Nonostante il complesso concetto sia crowded out, si cercano titoli per una tassonomia.

Lo stato della scienza è, tuttavia, non incoraggiante.

$\mathrm{Si}$ risolvono in definizioni tautologiche ${ }^{86}$ quelle che utilizzano i concetti di «rafforzamento, irrobustimento, rassodamento del sistema democratico", pur attribuendo un carattere teleologico minimo, «diretto ad aumentare la capacità di persistenza [del regime] e a contrastare e prevenire possibili crisi». ${ }^{87}$

Nonostante la deprecabile indeterminatezza del concetto, si registra una ormai asettica tendenza ad aderire alla "only game in town-definition" di Juan José Linz. ${ }^{88} \mathrm{La}$ formula dell'Autore è citata anche da chi, rivolgendosi alla costruzione di modelli che a parole si pretenderebbero diversi, muove i primi passi dallo smantellare questo o quel punto della posizione dottrinale di Linz. ${ }^{89}$

La dottrina sembra prendre comunque le mosse dalle considerazioni di Juan José Linz. Chi vi aderisce pedissequamente, chi s'avventura in approcci «innovativi», che esaltano un tratto della definizione di Linz, salvo poi ripiegare ma senza ammetterlo esplicitamente sulla definizione

85 Mezzetti, L., Le Democrazie Incerte. Transizioni costituzionali e consolidamento della democrazia in Europa orientale, Africa, America Latina, Asia, op. cit.. Diamond parla invece di «twilight zone» con riguardo alle democrazie elettorali che sono in una condizione di persistenza senza legittimazione o istituzionalizzazione. Cfr. Diamond L., Developing Democracy: Toward Consolidation, Baltimore, 1999, passim.

86 "Il consolidamento democratico è una condizione dei nuovi regimi democratici in cui [sia i fattori istituzionali che quelli culturali rendono] la democrazia sicura e stabile e, pertanto, regressioni autoritarie divengono improbabili» : Menéndez Moreno A., Political Cleavages. Issues, Parties and the Consolidation of Democracy, Boulder-Oxford, 1999, p. 2. In seguito, l'autore ridefinisce il consolidamento come «un processo», p. 19.

87 Sono parole di Morlino L., "Consolidamento democratico: definizione e modelli", in: Rivista Italiana di Scienza Politica, vol. 16.2, 1986, p. 203.

88 Grosso modo, "Nessun attore politico considera percorribili alternative alla democrazia".

Occorrebbe introdurre sfumature in tale non indiscriminata accettazione. L'ordinamento che prontamente non pone in marcia riforme sociali e socio-economiche evidenti sarà sfidato dal discontento delle masse. Vedi Alcántara M., «De la reforma y la consolidación del sistema político en el equilibrio entre democracia y mercado en América Latina", in Alcántara M. e Crespo I., (a cura di), Los limites de la consolidación democrática en América Latina, Salamanca, 1999, pp. 29-45.

89 Ad esempio Guillermo O'Donnell o Joe Foweraker. 
in toto, recuperandola. Che la dottrina si sia adattata ed adagiata non è un fattore positivo, data la poliformità del concetto e delle applicazioni ancora tutte da mappare. E nuovi utilizzi —occorrono metri sicuri, ma ogni volta convalidati per potere avallarli-a talora disinvolti si fanno continuamente strada.

La definizione di Linz che è dominio pubblico è definizione minimalista nel senso di Sartori, ${ }^{90}$ svicolata da tratti propri di un particolare modello di democrazia (quella nord-occidentale, in specie) e pertanto capace di plasmarsi, adattandosi ai più disparati contesti culturali, ad ogni Paese del mondo. Non così per le concezioni massimalistiche.

$\mathrm{E}$ ancora, dietro l'apparente appagamento, apparente uniformazione nell'accettazione dell'itinerario di Linz, in realtà accade che gli studiosi si riferiscano a fenomeni empirici distinti. ${ }^{91}$ Per Munck la confusione è stata aggravata dall' utilizzo delle già divergenti concezioni per affermazioni causali. ${ }^{92}$

Burton, Gunther e Higley firmano la resa: la democrazia consolidata è un idealtipo. ${ }^{33}$ E giacchè nessun ordinamento democratico soddisfa appieno i requisiti, non sarà mai completamente consolidato nel senso dell'idealtipo, si preferisce guardare al consolidamento come ad un "processo di adattamento-congelamento di strutture e norme democratiche che vengono accettate come legittime da parte o dall'intera società civile» (Morlino, 1986) ${ }^{94} \mathrm{o}$, ancora, come un processo attraverso il quale le forme democratiche vengono valutate per sè stesse, anche contro risultati sostanziali avversi (Bolivar Lamounier, 1988). In un'ottica dinamica, il consolidamento è il processo di conversione dei modelli in strutture (Schmitter, 1988). ${ }^{95}$

90 Sartori G., "Concept Misformation in Comparative Politics", in: American Political Science Review, vol. 64.4, 1970.

91 Per tutti, Schedler, A., "What is Democratic Consolidation?", in: JoD, 9.2, 1998, pp. 103-104.

92 Munck, G. L., Democracy Studies: Where to From Here?, Urbana-Champaign [III], 2001 .

93 Burton M., Gunther R. e Higley J., "Introduction: elite transformations and democratic regimes», in: Higley J. e Gunther R. (a cura di), Elites and Democratic Consolidation in Latin America and Southern Europe, Cambridge, 1992, pp. 1-37.

94 Morlino, L., "Consolidamento democratico: definizione e modelli», in: Rivista Italiana di Scienza Politica, vol. 16.2, 1986, p. 210.

95 Schmitter P. C., "Interest Systems and the Consolidation of Democracies", in: Marks G. e Diamond L. J., (a cura di), Reexaming Democracy. Essays in Honor of Seymour Martin Lipset, Newbury Park, 1988, p. 158. 
Resta da vagliare, entro queste prime approssimazioni, se il consolidamento sia un processo o una situazione, o, ancor più probabilmente, ambedue le cose. ${ }^{96}$ Morlino non ha dubbi sul carattere procedimentale del concetto, ${ }^{97}$ carattere che implica estensione temporale e interrelazioni tra la variabile tempo e ciascuno degli elementi essenziali del sistema politico.

I processi di consolidamento possono differire profondamente da caso a caso: ogni contesto storico può coinvolgere differenti istituti e attori a stadi divergenti del singolo processo; può variare notevolmente l'ammontare di tempo richiesto per consolidare l'ordinamento; i progressi verso il consolidamento non sono nè inevitabili, nè uni-lineari. ${ }^{98}$

Plasser, Ulram e Waldrauch (1997), ${ }^{99}$ nel tentativo di far combaciare l'idea di democrazia come never-ending process ${ }^{100}$ con l'intuizione di un assetto permanente che il consolidamento porta con sè, spiegano che il consolidamento non è necessariamente un processo lineare, unidirezionale, monoconducente, nè unidimensionale. «Il consolidamento di un regime politico può soltanto aumentare le probabilità per la sua sopravvivenza, ma non può mai interamente eliminare la possiibilità di caduta o collasso».

Di diverso avviso, almeno quanto ad enunciazioni, è Linz: la democrazia consolidata è una "political situation», ${ }^{101}$ uno state of being. E' chiaro,

96 Ciò darebbe origine al carattere teleologico del concetto. Pare che ciò non sia bene e vada rimediato

a- considerando la democrazia consolidata (the final destination) un ideal-tipo e concentrandosi sul processo. Così, ad es. Valenzuela o Linz e Stepan; ovvero

b- separando chiaramente il prodotto ultimo di lungo termine del consolidamento (la sostenibilità democratica) dal concetto di consolidamento stesso. In questo senso Gunther, Puhle e Diamandouros.

97 Cfr. anche Valenzuela, J. S., Democratic Consolidation in Post-Transitional Settings: Notion, Process, and Facilitating Conditions, Kellogg Institute, Working Paper No 150, dicembre, 1990.

98 Gunther, R., Puhle, H.-J. e Diamandouros N., «Introduction», in: Gunther R., Diamandouros N. e Puhle H.-J. (a cura di), The Politics of Democratic Consolidation. Southern Europe in Compatative Perspective, Baltimore-London, 1995, pp. 19-20.

99 Plasser F., Ulram P. A. e Waldrauch H., "The Concept of Consolidation in Regime Change Research", in: Democratic Consolidation in East-Central Europe, HoundmillsLondon, 1998, pp. 3-56.

100 "Democracy is not but is constantly becoming", nelle parole di von Beyme.

101 Linz, J. J. e Stepan, A., Problems of Democratic Transition and Consolidation. Southern Europe, South America, and Post-Communist Europe, Baltimore-London, 1996, p. 6. 
tuttavia, come i due autori stiano facendo riferimento a fattispecie diverse e come Linz si riferisca al punto d'arrivo del processo, quello che Morlino chiama «stabilità». ${ }^{102}$

\subsection{Serie di definizioni}

Al fine di non incorrere in pericolosi equivoci sarà bene tenere prudentemente distinti alcuni concetti. Va da sè che l'esposizione si regge sulla distinzione, fondante sotto questo profilo, tra transizione e consolidamento.

Altri termini potrebbero far cadere in errore. Ve ne sono di afferenti al campo perlomeno semantico del consolidamento (quali deeping e profundization), ${ }^{103}$ ve ne sono di peregrini e maldestramente accostati, talora confusi (ad esempio stabilità, durata). ${ }^{104}$

Alcuni concetti fuoriescono dall'ambito di significanza del consolidamento. Linstaurazione, intesa come delineamento delle nuove istituzioni e procedure democratiche, va meglio allocata in tempo di transizione. All'interno dell'instaurazione stessa si trova la strutturazione, la fase di formazione ed articolazione delle diverse strutture proprie del sistema politico.

Da trattare con cautela, secondo Morlino, il concetto sociologico di istituzionalizzazione: esso pertiene al processo di emersione delle struttureistituzioni e a quello del loro rafforzamento. Guillermo O'Donnell attribuisce, invece, un duplice significato, più contiguo al consolidamento, alla institutionalization: si tratta del processo di graduale stabilizzazione delle aspettative che un dato sistema adotta al fine di orientare le azioni politiche, ovvero della crescente aderenza tra istituzioni formali e pratiche reali.

Guillermo O'Donnell, osserva Diamond, ${ }^{105}$ ha intuito per primo la distinzione che deve intercorrere tra il consolidamento e quella che si ritiene

102 Morlino, L., Consolidamento democratico: definizione e modelli, op. cit., p. 200.

103 Schmitter afferma che il consolidamento è una specie del genere strutturazione, istituzionalizzazione, routinizzazione, stabilizzazione, reificazione. Si tratta di fenomeni consanguinei.

104 Una cosa è il consolidamento di una democrazia , altro è la sua stabilità.

105 Diamond, L., Developing Democracy: Toward Consolidation, Baltimore, 1999, p. 65 e 301 ; Diamond L., "Rethinking Civil Society: Toward Democratic Consolidation», in: JoD, vol. 5.3, 1994, p. 15. 
essere la sua principale conseguenza (l'esito, l'accadimento che chiude il processo): la stabilità e persistenza della democrazia.

Venendo a concetti successivi al consolidamento, con stabilità Ismael Crespo indica la capacità di preservare procedimenti minimi di partecipazione e competizione. La stabilità si ottiene anche se sussiste volontà di non inglobare attori e temi nell'arena politica.

La persistenza democratica, quale risultato finale del processo di democratizzazione, attiene alla capacità del regime di sopravvivere, di aggiustarsi e di resistere alla sfide più severe. ${ }^{106}$ Il consolidamento contribuisce alla capacità di persistere dell'ordinamento, ma in nessun senso il consolidamneto conseguito ad un certo punto può garantire che un particolare regime sopravviva indefinitamente. ${ }^{107}$ Crespo opta per una linea più ambigua: se la persistenza è l'eliminazione dei rischi di regresso autocratico, allora un regime può dirsi persistere pur se incapace di gestire l'incertezza istituzionale, se incapace di efficacemente condurre la gestione statale. In quest'ottica, se il tentativo di consolidamento ha conosciuto un collasso, c'è spazio per un regime permanente di crisi ovvero per una persistenza instabile. ${ }^{108}$ Il consolidamento proseguirebbe senza soluzioni

106 Diamandouros, N. e Gunther, R., "Preface», in: Gunther R., Diamandouros N. \& Puhle H.-J. (a cura di), The Politics of Democratic Consolidation. Southern Europe in Compatative Perspective, Baltimore-London, 1995, pp. ix-xxx.

Schmitter parla della "persistenza delle democrazie inconsolidate" sostenendone la non regressione in autocrazie (concetto da alcuni denominato "consolidamento negativo") : Schmitter P. C., "Consolidation", in: Lipset S. M. (a cura di), The Encyclopedia of Democracy, I, 1995, p. 299.

107 Diamandouros, N. e Gunther R., "Preface", in: Gunther R., Diamandouros N. e Puhle H.-J. (a cura di), The Politics of Democratic Consolidation. Southern Europe in Compatative Perspective, op. cit., pp. ix-xxx.

108 Morlino L., "Authoritarianism", in: Bebler A. e Seroka J., (a cura di), Contemporary Political Systems: Classifications and Typologies, Boulder, 1990, pp. 91-101. Il problema consiste nel fatto che molte nuove democrazie meramente sopravvivono senza consolidarsi. Cfr. Munck G. L., "Democratic Transitions in Comparative Perspective", in: $C P$, vol. 26, 1994, pp. 355-375.

Per Gerardo Munck, attualmente gli studi sono disposti a distinguere il processo di consolidamento da uno di eventuale deconsolidamento (con un arretramento della soglia di consolidamento), da uno di crisi e riequilibrio (con il fallimento di un insieme di regole), da uno di transizione mediante riconsolidamento. Vedi Munck G. L., Disaggregating Political Regime: Conceptual Issues in the Study of Democratization, Kellogg Institute, Working Paper No 228, New Haven, agosto, 1996. 
di continuità nella persistenza (Rosales preferisce parlare di normalità), più o meno stabile a seconda del grado di consolidamento raggiunto.

Mentre il consolidamento riguarda le nuove democrazie, si perfezionano (Dahl si esprime in termini di approfondimento) ${ }^{109}$ quelle già consolidate. ${ }^{100} \mathrm{Il}$ perfezionamento, a tale riguardo regna concordanza in dottrina, attiene alla realizzazione più completa di diritti, libertà e, più in generale, degli ideali democratici, in particolare alla estensione ad altri ambiti della vita sociale.

La qualità della democrazia, invece, va relazionata all'espansione della cittadinanza, cioè alla partecipazione, rappresentazione e soddisfazione dei cittadini mediante i processi decisionali a livello locale, regionale e centrale. ${ }^{111}$

L'efficacia è la capacità del sistema di progettare soluzioni ai problemi generati dal proprio stesso funzionamento, capacità di progettazione ed esecuzione degli affari statali. La forza dello Stato deriva piuttosto dal rispetto della legge garantito dal governo (Morlino).

$\mathrm{Al}$ concetto di democrazia in funzione accedono svariati altri concetti, quali rappresentatività-congelamento e governabilità-ingovernabilità. Il concetto di governabilità -particolarmente sentito in America Latina- viene latamente inteso come disimpegno governativo in termini di opportunità, effettività, accettazione sociale, efficienza (in particolare nel campo delle politiche economiche e dell'amministrazione della gestione pubblica) e coerenza delle decisioni (Flisfisch). ${ }^{112}$ Ancora, Morlino aggiunge

109 Dahl, R. A., On Democracy, New Haven, 1998; trad. it. Sulla democrazia, Bari, 2000, p. 4.

Manuel Antonio Garretón preferisce utilizzare l'espressione profundización democrática per indicare un miglioramento della democrazia, qualunque ne sia il contesto; tale uso vorrebbe indicare come il consolidamento, essendo un ideale, sia in realtà non raggiungibile.

110 Morlino, L., «Introduzione all'edizione italiana», in: Linz J. J. e Stepan A., Transizione e consolidamento democratico, Bologna, 2000, pp. vii-xvi.

111 Carretón, M. A., "Democratización, desarrollo, modernidad: ¿Una nueva problemática para América Latina?", in: Alcántara M. e Crespo I., (a cura di), Los límites de la consolidación democrática en América Latina, Salamanca, 1999, pp. 75-82.

112 Flisfisch, Á., "Gobernabilidad y consolidación democrática: sugerencias para la discusión", in: Revista Latinoamericana de Sociología, vol. 51.3, pp. 113-134; cfr. inoltre Camou A., Gobernabilidady Democracia. Elementos para un Mapa Conceptual, UACJ, Noesis 13 ; Feldman, E., "La evolución de la reflexión politológica sobre la democratización: del cambio de régimen a la arquitectura institucional de governance y la gobernabilidad", in: Biblioteca de ideas, Desarrollo institucional para una gobernabilidad democrática, No $8 / 9$, maggio 2001 . 
che con «incapsulamento" s'intende indicare il processo di contenimento dei conflitti mediante norme.

La modernizzazione, infine, pretende essere lo studio di come le istituzioni affettino valori ed outcomes individuali e collettivi. Trattando di salute, industrializzazione, educazione, urbanizzazione, benessere e forse istituzioni, ${ }^{113}$ esautora, come irrilevanti, la democratic performance, stability o consolidation e persino la sopravvivenza della democrazia.

\subsection{Le distinzioni dalla transizione}

$\grave{E}$ bene delineare in primo luogo il consolidamento come momento di relativizzazione: le passioni della transizione si rimodellano, si prestano a nuove dimensioni. Ciò non significa che il consolidamento sia un momento di stagnazione: i sentimenti (ri)vivono in nuovi attori e nuove direzioni che esordiscono sullo scenario non solo nazionale.

Se con transizione si intende la creazione delle istituzioni fondamentali e il delineamento delle nuove regole per la condotta politica di ogni attore e con consolidamento la legittimazione di tali istituzioni e l'interiorizzazione delle nuove norme democratiche di comportamento, allora transizione e consolidamento sono concettualmente distinti, ma possono sovrapporsi temporalmente e persino coincidere. ${ }^{114}$

Manuel Alcántara Sáez smentisce l'idea meccanicistica per cui inevitabilmente tutte le transizioni politiche son seguite da un processo di consolidamento. ${ }^{115}$

Intuitivamente, Gerardo Munck sottolinea la distinzione per cui nella transizione è in gioco l'emergere stesso delle regole, nel consolidamento

113 Johannsen, L., Democratization and Development: Modernization and Political Institutions, Århus-Copenhagen, 2000. Secondo l'Autore danese, le istituzioni possono studiarsi nella fase di creazione mediante la transitology e nella fase di funzionamento mediante la tcoria della Modernizzazione.

Sulla relazione tra teoria dela modernizzazione e consolidamento, Martí i Puig S., "¿Promesas incumplidas? Un balance critico de las teorias del cambio politico y su aplicabilidad en América Latina", in: Revista Instituciones y Desarrollo, No 10, 2001, pp. 201-235.

114 Diamandouros, N. e Gunther R., "Preface», in: Gunther R., Diamandouros N. e Puhle H.-J. (a cura di), The Politics of Democratic Consolidation. Southern Europe in Compatative Perspective, op. cit., pp. ix-xxx.

115 Alcántara, M., "De la reforma y la consolidación del sistema político en el equilibrio entre democracia y mercado en América Latina», in: Alcántara M. e Crespo I., (a cura di), Los límites de la consolidación democrática en América Latina, op. cit., p. 37. 
le regole sono date per esistenti. Nel primo caso si tratta dei problemi di cambio di regime, nel secondo di quelli di funzionamento del regime. ${ }^{116}$

Secondo Ismael Crespo la transizione è periodo di definizione di un ordine-le regole, norme e istituzioni del nuovo gioco democratico-, laddove il consolidamento tenta di ricomporre tale ordine: ristrutturare il sistema di articolazione tra società civile, sistema politico e Stato attorno al nuovo formato istituzionale definito e legalmente delimitato. La transizione è azione per determinare le regole, il consolidamento è azione sviluppata in un quadro normativo già delimitato. ${ }^{117}$

Per Schmitter i due processi impegnano «differenti attori, condotte, processi, valori e risorsen. ${ }^{118}$

Il consolidamento è molto più complesso e coinvolge un maggior numero di attori in un'arena politica allargata. In un'ottica soggettiva, ciascun processo è strutturato in modo da massimizzare (durante la transizione) o da porre forti vincoli (durante il consolidamento) alla libertà di movimento di cui godono gli attori coinvolti. ${ }^{119}$ Nelle democrazie consolidate tutti i principali attori politici danno per assodato il fatto che le procedure democratiche dettino il rinnovamento del governo; nelle «democrazie» in transizione $\mathbf{i}$ governi eletti operano in un ambiente politico in cui la continuità democratica è ancora incerta. Nella transizione prevale il sentimento d'euforia, nel consolidamento il desencanto. ${ }^{120}$ Parimenti con attenzione agli attori, ma con maggior sensibilità per le

116 Munck, G. L., Disaggregating Political Regime: Conceptual Issues in the Study of Democratization, op. cit.

117 Crespo, I., "¿Hacia dónde van las democracias latinoamericanas?", in: Alcántara M. e Crespo I., (a cura di), Los limites de la consolidación democrática en América Latina, op. cit., pp. 13-28.

118 Il consolidamento «non è solo un prolungamento della transizione». Se molti dei soggetti rimarranno "gli stessi", cambieranno i problemi cui si relazioneranno e il loro comportamento. V. Schmitter P. C., "Transitology: The Science or the Art of Democratization?", in: Tulchin J. S. e Romero, B. (a cura di), The Consolidation of Democracy in Latin America, Boulder, 1995, p. 12.

119 Diamandouros, N. e Gunther R., "Preface", in: Gunther R., Diamandouros N. e Puhle H.-J. (a cura di), The Politics of Democratic Consolidation. Southern Europe in Compatative Perspective, op. cit., pp. ix-xxx.

120 Mainwaring S., O’Donnell G. \& Valenzuela S., "Introduction», in: Mainwaring S., O'Donnell G. e Valenzuela S., (a cura di) Issues in Democratic Consolidations: The New South American Democracies in Comparative Perspective, Notre Dame, 1992, pp. $1-16$. 
inclinazioni, José M. Rosales registra come l'orientamento cooperativocostruttivo ceda il passo a un orientamento di segno cooperativo-competitivo, a misura in cui il pluralismo è incorporato alla dinamica istitutizionale e la mappa politica sperimenta riaggiustamenti ideologici e strategici. ${ }^{121}$ Schmitter in tal senso suole distinguere tra guerra di movimento, di difficile predittibilità, e guerra di posizione, nella quale gli attori si limitano e riconoscono mutuamente. ${ }^{122}$

Per Hyman, se in tempo di transizione la questione è l'agire umano, in tempo di consolidamento occorre focalizzare l'attenzione su incentivi e ostacoli all' 'istituzionalizzazione. ${ }^{123}$

Anche i prodotti dei due processi sono diversi: la transizione sfocia nella creazione di un nuovo regime; il consolidametno sfocia nella stabilità e persistenza di tale regime. Il punto finale di una transizione [in senso lato] può essere un regime non democratico; il consolidamento, invece, richiede piena aderenza ai criteri di una democrazia procedurale.

Per Gunther, Diamandouros e Puhle, diverse sono le variabili esplicative. ${ }^{124}$ Si comporteranno in modi diversi le variabili tralaticie: rilevanti per la transizione, potranno scolorare successivamente $o$, viceversa, potranno assumere rilevanza con l'avanzare del consolidamento (ad esempio: irrilevanza successivamente acquisita dalle condizioni vincolanti la transizione; emergere del ruolo del sostegno delle masse).

Il consolidamento è di più difficile delineazione rispetto alla transizione: ingloba, tra l'altro, un numero maggiore di livelli di attività. ${ }^{125}$ L'analista

121 Rosales, J. M., "Tras la consolidación democrática: la normalidad en la articulación entre el sistema de instituciones y una política civica", in: Alcántara M. e Crespo I., (a cura di), Los límites de la consolidación democrática en América Latina, op. cit., pp. 83-101.

122 Karl parla di «structured contingency": Karl T. L., "Dilemmas of Democratization in Latin America", in: $C P$, vol. 22.4, 1990, pp. 5-8.

123 Gerald F. Hyman G. F. (et. al.), Conducting a Democracy and Governance [DG] Assessment: A Framework for Strategy Development, Center for Democracy and Governance, U.S. Agency for International Development [USAID], Development Experience Clearinghouse [DEC], Document PN-ACG-505, Washington DC, dicembre, 1999.

124Gunther R., Nikiforos, P., Diamandouros e Puhle, H.-J., The Politics of Democratic Consolidation. Southern Europe in a Comparative Perspective, Baltimore-London, The Johns Hopkins University Press, 1998, p. 3.

125 Pridham G., "Introduction», in: Pridham G. (a cura di), Transitions to Democracy. Comparative Perspectives from Southern Europe, Latin America and Eastern Europe, Aldershot-Brookfield, 1995, pp. xi-xxv. 
deve slittare dalla "political causality" ad una forma di "bounded rationalitym. ${ }^{26}$

Va poi notato che la transizione può influire grandemente sul corso successivo del processo di consolidamento, ${ }^{127}$ tanto che Guillermo O'Donnell parla di «seconda transizione». ${ }^{128}$

Il consolidamento dura tendenzialmente più a lungo della fase che lo precede: si va dalla decade allo spazio di una generazione. ${ }^{129}$

Per Morlino, il consolidamento non presenta i caratteri di fluidità e creatività della transizione.

Ma è stato Adam Przeworski (1986) ad individuare nell'incertezza il tratto distintivo tra transizione e consolidamento. Incertezza che nel secondo processo è temperata da relative certezze. ${ }^{130}$

La fase della transizione è spesso di acuta incertezza e di alta ansietà per molti attori sociali. Progressivamente diminuiscono le tante insicurezze, al divenire le nuove procedure meglio note e capite e più fortemente accettate. Il nuovo regime diviene istituzionalizzato, le sue strutture di espressione politica aperta e competitiva vengono interiorizzate. ${ }^{131}$ Mentre

126 Schmirter P. C., "Transitology: The Science or the Art of Democratization?", in: Tulchin J. S. e Romero B. (a cura di), The Consolidation of Democracy in Latin America, op. cit., p. 12.

127 Gunther R., Puhle H.-J. e Diamandouros N., «Introduction», in: Gunther R., Diamandouros N. e Puhle H.-J. (a cura di), The Politics of Democratic Consolidation. Southern Europe in Compatative Perspective, op. cit., pp. 1-32.

128 «Il consolidamento della democrazia è l'effettivo funzionamento di un regime democratico": O'Donnell G., "Transitions, Continuities, and Paradoxes", in: Mainwaring S., O'Donnell G. e Valenzuela S., (a cura di) Issues in Democratic Consolidations: The New South American Democracies in Comparative Perspective, Notre Dame, 1992, pp. 17-56.

129 «Difficilmente il processo si compirà in meno di una generazione»: Whitehead L., "The Consolidation of Fragile Democracies: A Discussion with Illustrations", in: Pastor R. A. (a cura di), Democracy in the Americas: Stopping the Pendulum, New York-London, 1989, pp. 76-95.

130 Przeworski A., "Some Problems in the Study of the Transition to Democracy", in: O'Donnell G., Schmitter P. C., Whitehead L. (a cura di), Transition from Authoritarian Rule: Prospects for Democracy, vol. III, Baltimore, 1986, pp. 57-61.

131 Whitehead L., "The Consolidation of Fragile Democracies: A Discussion with Illustrations", in: Pastor R. A. (a cura di), Democracy in the Americas: Stopping the Pendulum, op. cit., pp. 76-95. 
la politica nel suo complesso si affranca dalla contingenza di elevato rischio propria della transizione, il comportamento politico trova una sua «strutturazione». ${ }^{132}$ Con le parole di Larry Diamond, l'impegno profondo, indiscusso e routinario alla democrazia e ai suoi procedimenti produce una riduzione dell'incertezza, cruciale nel consolidamento, non tanto per i risultati, quanto piuttosto per le regole e metodi di concorrenza politica. ${ }^{133} \mathrm{E}$ ' il grado d'incertezza ciò che differenzia le due fasi: l'assenza di prevedibili "regole del gioco" durante la transizione aumenta lo spazio politico per scelte contingenti. In quest'ottica, il consolidamento si ha allorchè le classi sociali e i gruppi politici in competizione arrivano ad accettare un insieme di regole formali o d'intese informali che determinino "who gets what, where, when, and how» in politica. ${ }^{134}$

\subsection{La sequenza del consolidamento}

L'intuizione di Philippe Schmitter (1995) è che il consolidamento potrebbe essere concepito come avente inizio prima che la stessa transizione sia finita. ${ }^{135}$ Geoffrey Pridham (2001) chiarisce che il consolidamento è, ad ogni modo, un processo multidimensionale o multi-livello in cui differenti dimensioni possono svilupparsi a passi variabili e,

132 Laddove nella transizione prevalgono casualità politiche in una situazione in rapido mutamento, elevato rischio e scelte strategiche indeterminate, durante il consolidamento gli attori organizzano le loro strutture interne in modo più previsibile, consultano l'elettorato più regolarmente, mobilitano più sicuramente le loro risorse di base, considerano più seriamente le conseguenze a lungo termine delle loro azioni e generalmente sperimentano più intensamente le costrizioni imposte da deficienze materiali profonde e abitudini normative. La casualità si sposta verso un fondamento socio-economico condizionato da relazioni di classe capitalistiche, spaccature culturali e etniche profonde, persistenti conflitti di status e antagonismo internazionale. Cfr. Schmitter P. C., "The Consolidation of Political Democracies: Processes, Rhythms, Sequences and Typesm, in: Pridham G. (a cura di), Transitions to Democracy. Comparative Perspectives from Southern Europe, Latin America and Eastern Europe, op. cit., pp. 535-569.

133 Diamond L., Is the Third Wave of Democratization over? The Imperative of Consolidation, Kellogg Institute, Working Paper No 237, marzo 1997.

134 Così Berntzen E., "Democratic consolidation in Central America: a qualitative comparative approach», in: Third World Quaterly, vol. 14.3, 1993, pp. 589-604.

135 Schmitter P. C., "The Consolidation of Political Democracies: Processes, Rhythms, Sequences and Types", in: Pridham G. (a cura di), Transitions to Democracy. Comparative Perspectives from Southern Europe, Latin America and Eastern Europe, op. cit., p. 542. 
speculativamente, il consolidamento può essere raggiunto in diversi momenti temporali. Può concepirsi il consolidamento come in fieri ad uno o più livelli mentre la transizione è ancora in corso. ${ }^{136}$

Le tappe essenziali del consolidamento sono indicate in quattro:

1. l'inizio dell'attività legislativa. Il termine a quo è la convocazione della prima sessione regolare di un Parlamento eletto in modo libero e competitivo. ${ }^{137} \mathrm{Da}$ questa divengono virtualmente accessibili processi che definiranno la rappresentanza e le relazioni di potere; ${ }^{138}$

2. la stabilizzazione dei sistemi di rappresentanza. Ciò significa che è completa l'istituzionalizzazione di due canali di accesso: quello territoriale [per i partiti] e quello funzionale [per i gruppi d'interesse]; ${ }^{139}$

3. la rotazione [rotativismo] o il maggior riallineamento coalizionale dei partiti al potere;

4. il completamento della regolazione «interna» delle principali istituzioni, peculiari a ciascun Paese perchè correlate al grado di implementazione del principio democratico conseguibile dal singolo ordinamento. Sempre si rinvengono, comunque, le c.d. «istituzioni democratiche di base»: un sistema partitico e uno dei gruppi d'interesse, un Parlamento funzionante e un Esecutivo responsabile. Centrale nel

136 Pridham, G., "Comparative reflections on democratisation in East-Central Europe: a model of post-communist transformation?", in: Pridham G. e Ágh A., (a cura di), Prospects for democratic consolidation in East-Central Europe, Manchester-New York, 2001, p. 4.

137 Schmitter P. C., "The Consolidation of Political Democracies: Processes, Rhythms, Sequences and Types", in: Pridham G. (a cura di), Transitions to Democracy. Comparative Perspectives from Southern Europe, Latin America and Eastern Europe, op. cit., pp. 535-569.

138 Che il Parlamento si riveli debole e non riesca a controllare la normazione necessaria, non implica che il regime sia meno democratico, quanto piuttosto democratico in un altro senso.

139 Il canale politico si ritiene stabile se l'identificazione partitica è elevata; se il reclutamento di militanti e candidati avviene secondo norma; se le preferenze dei votanti variano entro margini previsibili; se sono diminuite le differenziazioni regionali; se la defezione a favore di altro partito è cosa rara.

Il canale funzionale stabile dovrebbe avere volatilità contenuta; membership non erratica; volontà di unirsi tra gruppi preesistenti; omogeneità di accordi per area, tematica e momento; i cambiamenti locali divengono norme nazionali.

Tuttavia, di fronte a canali politici stabili è dato riscontrare un canale associazionale smembrato, sfilacciato. 
radicamento delle strutture è il processo di conversione [Verrechtlichligung] di pratiche e regole in norme formali sostenute da autorità statali. A questo stadio può considerarsi completo e terminato il consolidamento. ${ }^{140}$

Se Guillermo O'Donnell si rivela tautologico sulla sequenza, ${ }^{141}$ Ismael Crespo afferma che se il punto di partenza è univoco-il momento in cui è posto in funzionamento un accordo sulle nuove regole del gioco democratico-, assai complesso è individuare il punto d'arrivo e poter distinguere pertanto il processo di consolidamento dal funzionamento democratico routinario. ${ }^{142}$

Una democrazia consolidata, per Laurence Whitehead, è quella in cui le forze rivali hanno un comune senso di authorship e investono assieme sul futuro. Un sentimento di tale tipo può radicarsi unicamente a mezzo di negoziati tra antagonisti. Tanto deve cambiare la percezione che gli attori hanno di sè, degli altri e del sistema nel suo complesso che, forse, il miglior test per appurare quando il processo di consolidamento ha raggiunto la sua conclusione consiste nell' individuare il momento a partire dal quale la storia della transizione può esser dibattuta senza temerne le conseguenze. ${ }^{143}$

140 Il consolidamento ha termine quando le istituzioni democratiche di base hanno acquisito uno status «esterno" stabile e hanno regolato le loro strutture «interne». Tali istituzioni devono esser sufficientemente radicate (vale a dire: protette da sè stesse e da altri interessi nonchè dotate di significato simbolico e approvazione normativa) così da resistere a possibili cambiamenti ambientali. Vedi Schmitter P. C., The Consolidation of Political Democracies: Processes, Rhythms, Sequences and Types, op. cit., pp. 544-545.

$141 \mathrm{La}$ fase del consolidamento si apre con l'inaugurazione di un governo democratico e termina con lo stabilimento di un regime democratico consolidato. Cfr. O'Donnell G., "Transitions, Continuities, and Paradoxes", in: Mainwaring S., O'Donnell G. \& Valenzuela S., (a cura di) Issues in Democratic Consolidations: The New South American Democracies in Comparative Perspective, op. cit., pp. 17-56.

142 Distinzione che, aggirando l'etichetta "routinario", è a tal punto sottovalutata da O'Donnell (1992), da esser sovvertita in definizione (il consolidamento $\grave{e}$ il funzionamento democratico). Cfr. Crespo I., "Hacia dónde van las democracias latinoamericanas?", in: Alcántara M. e Crespo I., (a cura di), Los limites de la consolidación democrática en América Latina, op. cit., pp. 13-28.

143 Whitehead, L., "The Consolidation of Fragile Democracies: A Discussion with Illustrations", in: Pastor R. A. (a cura di), Democracy in the Americas: Stopping the Pendulum, op. cit., pp. 76-95. 


\section{Approcci dottrinali}

Il processo di consolidamento può essere analizzato lungo una duplice prospettiva ${ }^{144}$ minima di fondo:

a. attiva: il consolidamento è visto come l'attiva creazione di istituzioni democratiche o la rimozione di quelle «autoritarie»;

b. evolutiva: il consolidamento è visto come un processo di adattamento in cui politici e cittadini imparano ad agire secondo le regole democratiche. L'accento è posto sul graduale cambiamento di valori, quali la fiducia e la cultura civica. In questa seconda ottica, il consolidamento deve avvenire a quattro distinti livelli: ideologico, istituzionale, di società civile e culturale (Fukuyama). ${ }^{145}$

Non si tratta tuttavia delle uniche interpretazioni possibili.

Variamente gli Autori considerano centrale il ruolo delle elezioni e delle procedure in genere, ovvero delle istituzioni; il ruolo dei soggetti (non solo delle élite) e delle loro inclinazioni, la questione del consenso; il ruolo (talora letto come esclusivo) dei fattori sociali o dei fattori economici. $^{146}$

In genere si lamenta evanescenza, confusione, difficoltà a comunicare. ${ }^{147}$ E come rimedio, si tenta di acclarare semanticamente il concetto di consolidamento democratico ovvero ripudiarlo.

144 Boussard C., "Civil Society in the Consolidation Process. Illustrations from Central America", ECPR 28 Joint Sessions of Workshops, Workshop 4, Copenhagen, aprile, 2000.

145 Fukuyama, F., "The Primacy of Culture», in: JoD, vol. 6.1, 1995, pp. 7-14.

146 Chiaramente, la concezione di Linz non è l'unica mai claborata, pur avendo messo in ombra ogni altra. Un certo itinerario evolutivo si è dovuto percorrere da parte dello stesso Linz e da tutta la dottrina, necessariamente, per giungere ad una formulazione così largamente accolta.

147 Vedi Gunther R., Puhle H.-J. e Diamandouros N., "Introduction", in: Gunther R., Diamandouros N. e Puhle H.-J. (a cura di), The Politics of Democratic Consolidation. Southern Europe in Compatative Perspective, op. cit., p. 5.

Cfr. altresì Schedler A., Concepts of Democratic Consolidation, Latin American Studies Association (LASA), Wien-Guadalajara, aprile, 1997; in seguito "What is Democratic Consolidation?", in: JoD, vol. 9.2, 1998, pp. 91-107. 


\subsection{Concezione soggettiva-psicologica}

\subsubsection{Concezione negativa}

La costruzione di Evelyne Huber, Dietrich Rueschemeyer e John D. Stephens, nell'ottica sociologica della relazione tra Stato e classi sociali, dà una lettura in negativo del consolidamento, intendendolo quale assenza di pericoli per la democrazia $\mathrm{o}$, più correttamente, come assenza di classi antidemocratiche. ${ }^{148}$

A ben vedere, la riduzione degli attori antisistema non è che un aspetto, assai parziale, della concezione minimalista. Con questo si vuole semplicemente sostenere che l'angolo prospettico proposto dai tre Autori è eccessivamente angusto $e$ in nessun modo può coprire l'estensione di un concetto complesso come il consolidamento democratico.

\subsubsection{Concezione positiva}

Il riferimento è a Dankwart Rustow (1969) e alla habituation phase. ${ }^{149}$ Il rilievo fondante è che una decisione sgradevole è più accettabile se si è forzati a vivere con essa.

Con la sua pratica di dibattito multilaterale, i principi di conciliazione e aggiustamento, la democrazia implica un processo di collaudo ed errori, un'esperienza congiunta di apprendimento.

Nella fase di abitudine si innescano tre processi:

1. politici e cittadini imparano dalla positiva soluzione di alcune questioni a porre fede nelle nuove regole e ad applicarle a nuove tematiche;

148 Cfr. le opere di Rueschemeyer D., Huber Stephens E. e Stephens J. D., Capitalist Development and Democracy, Chicago-Cambridge, 1992; "The Paradoxes of Contemporary Democracy. Formal, Participatory, and Social Dimensions", in: CP, vol. 29, 1997, pp. 323342.

Per un commento, Remmer K. L., "New Theoretical Perspectives on Democratization», in: $C P$, vol. 28.1, 1995, pp. 103-122. Per un approfondimento della visione prospettata, Huber E., Ragin C. e Stephens J. D., «Social Democracy, Christian Democracy, Constitutional Structure and the Welfare Staten, in American Journal of Sociology, vol. 99, 1993, pp. 711749 ; Huber E. \& Stephens J. D., “Internalization and the Social Democratic Model», in: Comparative Political Studies, 1997.

149 Rustow D. A., "Transition to Democracy: Toward a Dynamic Model", in: CP, vol. 3, 1970, pp. 337-363, in particolare 358-361. 
2. la sperimentazione delle tecniche democratiche e il reclutamento competitivo confermerà i politici nelle loro pratiche e credenze;

3. la popolazione diverrà fermamente adattata alle nuove strutture mediante la forgiatura di efficaci legami di partito che riconnettono i politici nella capitale con l'elettorato disperso nel Paese.

Laurence Whitehead (1989) individua, con il progredire del consolidamento, un allargamento della gamma di attori politici che arrivano a presumere una condotta e una lealtà democratica nei propri avversari; un passaggio dagli impegni democratici strumentali a quelli di principio; un aumento della fiducia e della cooperazione tra politici concorrenti; una socializazione della popolazione in generale, a mezzo di sforzi deliberativi e della pratica della democrazia nella politica e nella società civile. ${ }^{150}$

Collaborando tra loro, le élite partitiche garantiscono vitali processi decisionali e organizzano, moderano, controllano la partecipazione e legittimazione a livello di massa, integrando nuovi gruppi sociali. Il rafforzamento del sistema dei partiti coincide con il consolidamento. ${ }^{151}$

Rafael del Águila pensa al consolidamento in termini -interattivi- di "assunzione di regole da parte degli attori", assunzione accompagnata dall'attribuzione di identità distinte e dipanata in un contesto in cui non esistano valide alternative di regime percorribili. ${ }^{152}$

Gerardo L. Munck ${ }^{153}$ definisce il consolidamento (in quella che successivamente chiamerà "definizione in senso ampio") ${ }^{154}$ come il periodo che si apre quando un insieme di regole del gioco politico è già stato

150 Quelle enunciate sono indicate dall'Autore come condizioni necessarie a che il consolidamento inizi, non tanto al consolidamento, nè al suo buon esito. Whitehead L., "The Consolidation of Fragile Democracies: A Discussion with Illustrations", in: Pastor R. A. (a cura di), Democracy in the Americas: Stopping the Pendulum, op. cit., pp. 76-95.

151 In questi termini, Grassi D., La Democrazia in America Latina. Problemi e prospettive del consolidamento democratico, Milano, 1999, pp. 21-22.

152 Del Águila R., "La transformación de las relaciones políticas. Presentación", in: Alcántara M. e Crespo I., (a cura di), Los límites de la consolidación democrática en América Latina, op. cit., pp. 69-74.

153 Munck, G. L., Disaggregating Political Regime: Conceptual Issues in the Study of Democratization, Kellogg Institute, Working Paper No 228, New Haven, agosto, 1996.

154 Munck, G. L., "Democratic Consolidation», in: Clarke P. B. e Foweraker ]. (a cura di), Encyclopedia of Democratic Thought, London-New York, 2001, pp. 175-178. 
definito e si chiude quando tali regole cessano di essere operative. "Il consolidamento delimita un processo che dipende interamente da una questione analitica: l'accettazione o il rigetto da parte dei soggetti di tali regole [già] stabilite».

\subsubsection{Imperniata sul comportamento}

Philippe C. Schmitter cristallizza il proprio pensiero in termini soggettivi ritenendo che il consolidamento importi la stabilizzazione, routinizzazione, istituzionalizzazione e/o legittimazione di modelli di comportamento politicamente rilevante. L'idea fondamentale è che le relazioni sociali possono divenire strutture sociali, ossia che modelli di interazione possono divenire così regolari nella loro frequenza, così impregnati di significato, così capaci di motivare comportamenti, da diventare autonomi nella loro funzione interna e resistenti ai cambiamenti indotti dall'esterno.

Fritz Plasser, Peter Ulram e Harald Waldrauch, ${ }^{155}$ nel descrivere la behavioral dimension del consolidamento, richiedono lo stabilizzarsi di pratiche politiche conformi ai principi democratici fondamentali e, al contempo, l'eliminazione, neutralizzazione o isolamento di ogni condotta che contribuisca a minare la democrazia. ${ }^{156}$

Secondo gli Autori tedeschi, quattro sono i modelli da considerare:

1. relazioni a livello di istituzioni governative;

2. relazioni d'influenza tra società (estremo attivo) e istituzioni governative (estremo passivo). Élite e masse devono persuadersi che le elezioni sono il canale «centrale ed inevitabile» d'influenza sull'attività di decisione politica;

3. relazioni d'influenza tra istituzioni governative (estremo attivo) e società (estremo passivo). Ciò implica la concreta implementazione e il rispetto dei diritti e libertà fondamentali ;

4. relazioni intrasociali, giacchè [sempre] si riscontrano nel tessuto sociale attori politici che tentano di ostacolare le organizzazioni sociali e di bloccare l'accesso di alcuni ai processi decisionali e alle istituzioni.

155 Plasser, F., Ulram P. A. e Waldrauch H., "The Concept of Consolidation in Regime Change Research", in: Democratic Consolidation in East-Central Europe, HoundmillsLondon, 1998, pp. 3-56.

156 Arrivando a prospettare -per eccesso-l'assenza di gruppi organizzati che tentino di minare [erodere o rovesciare] la democrazia. 


\subsubsection{Imperniata sull'atteggiamento}

Plasser, Ulram e Waldrauch ${ }^{157}$ indicano una dicotomia nell'interpretazione della attitudinal dimension del consolidamento: per taluni Autori è la legittimazione la variabile-chiave del consolidamento (Merkel) o l'unica variabile decisiva (Diamond); per altri è anche necessario lo sviluppo di una cultura politica (Pridham, Diamond).

\subsection{La legittimazione}

Per Larry Diamond ${ }^{158}$ il consolidamento è il processo di acquisizione di una larga e profonda legittimazione, ${ }^{159}$ tanto che ogni attore politico ritiene che il regime democratico sia il più giusto ed appropriato per la sua società, comunque migliore di ogni altra alternativa che si possa realisticamente prospettare. Ciò vale per le élite politiche, che devono persuadersi che le regole democratiche siano la sola strumentazione per governare e per i propri interessi, e deve valere per le masse, tagliando trasversalmente qualsivoglia frattura di classe, etnica etc., masse che devono arrivare ad un ampio consenso normativo e di comportamento sulla legittimità del sistema costituzionale, per povero e insoddisfacente che sia il suo disimpegno in un dato momento. Riassumendo, "la democrazia è consolidata soltanto quando nessun soggetto rilevante sfida la legittimità delle istituzioni democratiche o sistematicamente viola le sue norme costituzionali, le sue procedure e le sue leggi.»

Diane Ethier (1990) ${ }^{160}$ lega indissolubilmente il consolidamento delle democrazie allo sviluppo di un «consenso sociale». L'adesione ai valori

157 Plasser, F., Ulram P. A. e Waldrauch H., «The Concept of Consolidation in Regime Change Research", in: Democratic Consolidation in East-Central Europe, HoundmillsLondon, 1998, pp. 24-34 e 45.

158 Diamond, L., Developing Democracy: Toward Consolidation, op. cit., pp. 64116 e 301-316. Cfr. altresi, del medesimo Autore, Is the Third Wave of Democratization over? The Imperative of Consolidation, Kellogg Institute, Working Paper No 237, marzo 1997 ; «El final de la tercera ola y el futuro global de la Democracia», in: López E., Mainwaring S. (a cura di), Democracia: discusiones y nuevas aproximaciones, Buenos Aires, 2000, p. 72.

159 Tale legittimità, secondo Linz e Stepan, deve esser ben più di un impegno alla democrazia in astratto: deve essere un condiviso impegno normativo e di condotta per le regole e pratiche specifiche del sistema costituzionale: la lealtà al regime democratico. Cfr. Linz J. J. e Stepan A., Transizione e consolidamento democratico, op. cit., passim.

160 Ethier, D., "Introduction: Processes of Transition and Democratic Consolidation: 
democratici può esser spiegata, nella sua fase embrionale, sull'onda dell'apertura economico-politica che ha interessato il mondo nel secondo dopoguerra, ovvero per la modernizzazione delle strutture economiche e sociali che ha interessato l'Occidente attorno agli anni sessanta, ovvero infine- con la mera valutazione fatta da ogni singolo individuo sul potenziale del sistema democratico di soddisfare le proprie aspirazioni, bisogni e interessi.

Geoffrey Pridham -pur propendendo verbalmente per soluzioni massimaliste- risolve il contenuto del consolidamento nel processo di acquisizione della legittimazione del regime e, con esso, di una riformulazione della cultura politica (la «democratizzazione dei valori»). ${ }^{161}$ Il che significa rimozione delle prospettive per alternative di regime [negative consolidation] e adesione psicologica alla democrazia da parte di élite e pubblico in genere [positive consolidation]. ${ }^{162}$

Gerard Alexander, ${ }^{163}$ in termini di sostegno promesso o certo e in relazione all'Europa occidentale, definisce "consolidate» le democrazie in cui pressochè tutti gli attori hanno preferenze prodemocratic quanto a regime,

Theoretical Indicators", in: Ethier, D. (a cura di), Democratic Transition and Consolidation in Southern Europe, Latin America and Southeast Asia, Houndmills-London, 1990, pp. 3-21.

161 La graduale rimozione delle incertezze che affettavano la transizione apre la strada all'istituzionalizzazione delle nuove democrazie, all'interiorizzazione di regole e procedure e alla disseminazione di valori democratici. Per l'Autore, più correttamente, al profilo soggettivo di legittimazione a livello sia di élite che di massa, si accompagna la risoluzione delle eredità passate e delle sfide attuali, viste come costituenti ostacolo all'adesione ai valori e procedimenti democratici.

Cfr. Pridham G., "Introduction", in: Pridham G. (a cura di), Transitions to Democracy. Comparative Perspectives from Southern Europe, Latin America and Eastern Europe, op. cit., pp. xii e xxi-xxii.

Senza variazioni di formulazione, cfr. Pridham G., "Comparative reflections on democratisation in East-Central Europe: a model of post-communist transformation?", e "Rethinking regime change theory and the international dimension of democratisation: ten years after in EastCentral Europen, in: Pridham G. e Ágh A., (a cura di), Prospects for democratic consolidation in East-Central Europe, op. cit., pp. 1-24 e pp. 54-94.

162 Pridham G., "Introduction», in: Pridham G. (a cura di), Transitions to Democracy. Comparative Perspectives from Southern Europe, Latin America and Eastern Europe, op. cit., p. xxi.

163 Alexander, G., "Predictability and Democratic Consolidation", in: The Sources of Democratic Consolidation, Ithaca, 2002, pp. 56-89. 
opzioni che è altamente improbabile siano rovesciate come risultato di eventi routinari. La nozione è contrapposta alle uncertain democracies, ${ }^{164}$ parametrate in base alla predittibilità. Occorre che gli attori di destra percepiscano la democrazia come predictably la miglior opzione, rispetto a spinte centrifughe o implosive e percepiscano le sinistre come cambiate: non più meramente tatticamente moderate, ma forze previsibili.

\subsection{La cultura politica}

Per Plasser, Ulram e Waldrauch è stato da subito chiaro come il consolidamento "richieda cambiamenti a livello (inter)soggettivo" sia nel comportamento che nell'atteggiamento e, nell' attitudinal dimension, sia a livello di legittimazione che di cultura politica. Anche se fosse generalmente legittimato, un regime democratico potrebbe essere minacciato da «un piccolo gruppo» ed elevati margini di legittimazione non implicano necessariamente che gli attori siano capaci di comportamenti compatibili con la sopravvivenza della democrazia.

Larry Diamond ha elaborato in un secondo tempo ${ }^{165}(1998-2001)^{166}$ un coerente e sistematico inquadramento della «cultura politica». A suo avviso l'intima connessione intercorrente tra legittimazione e consolidamento rimarrebbe di gran lunga dominante nello spazio sociale, ponendo per la cultura politica questioni in rapporto a concetti ulteriori quali l'approfondimento della democrazia. Le inclinazioni culturali soggiacenti paiono meramente rallentare $o$ accelerare il consolidamento:

164 Democrazie in cui la preferenza di regime non è prodemocratic, o, pur essendolo, è passibile di essere soverchiata nel futuro prevedibile ad opera di eventi routinari quali sono le elezioni.

165 Non manca di rivendicare uno studio presentato con Alex Inkeless nel 1980. Si trattasi di Inkeless A. e Diamond L., "Personal Qualities as a Reflection of Level of National Development, in: Andrews F. e Szalai A., (a cura di), Comparative Studies in the Quality of Life, London, 1980, pp. 73-109; si ricordi inoltre il coordinamento del volume collettaneo Political Culture and Democracy in Developing Countries, Boulder, 1993.

166 Diamond, L., Political Culture and Democratic Consolidation, Instituto Juan March, Centro de Estudios Avanzados en Ciencias Sociales [CEACS], Working Paper No 118, giugno, 1998 ; "El final de la tercera ola y el futuro global de la Democracia», in: López E., Mainwaring S. (a cura di), Democracia: discusiones y nuevas aproximaciones, Buenos Aires, 2000, pp. 13-102 ; How People View Democracy: Findings from Public Opinion Surveys in Four Regions, Stanford, 2001. 
livelli diffusamente bassi di fiducia, ad es., costituiscono un chiaro problema. ${ }^{167}$ Diamond sottolinea gli attributi dell'impegno, della partecipazione, della risposta, della fiducia, della tolleranza.

Francis Fukuyama (1995) ${ }^{168}$ definisce la cultura «un abito a-razionale ed etico trasmesso attraverso la tradizione». È l'aspetto più profondo $\mathrm{e}$ meno malleabile del consolidamento. Vi include fenomeni quali la struttura familiare, la religione, i valori morali, la coscienza etica, il senso civico e particolari costumi storici.

Ronald Inglehart ${ }^{169}$ si sofferma sull'impatto che "values, belief and skills» della popolazione intesa come massa hanno sui processi di democratizzazione. La stabilità democratica pare correlata a istituti che veicolano i self-expressed values di una società: la democrazia rendebbe felici, sani, tolleranti e fiduciosi e instillerebbe valori post-materialisti. ${ }^{170}$ Non è tuttavia sempre così. Basti pensare alla società russa dopo il 1991. Al contrario, il progresso economico influenza grandemente il raggiungimento di determinati livelli di cultura politica [anche mediante un florido welfare state]. In seconda battuta, determinanti sono il ruolo delle élite e dei fattori internazionali.

\subsection{La concezione procedimentale [c.d. istituzionale]}

Si trattasi di quello che Gerardo Munck (2001) chiama "concetto di consolidamento in senso stretton: l'istituzionalizzazione dei processi elettorali inaugurati al termine della transizione e il loro mantenimento, in termini di durevolezza ed efficacia. ${ }^{171}$

167 Il presupposto della scuola tradizionale della transizione è la capacità di discernimento, attribuita ai cittadini, tra democrazia come valore intrinsecamente apprezzabile e performances del governo democratico. Essi sarebbero in grado di valutare valida l'opzione democratica anche quando percepiscano i risultati di maldestri disimpegni dello Stato, particolarmente in campo economico.

168 Fukuyama, F., The Primacy of Culture, op cit., pp. 7-14.

169 Inglehart, R. F., "Political Culture», in: Clarke P. B. e Foweraker J. (a cura di), Encyclopedia of Democratic Thought, op. cit., pp. 521-526.

170 "Adopt democratic institutions and live happily ever aftem.

171 Fortemente critico, Munck, G. L., "Democratic Consolidation", in: Clarke P. B. e Foweraker J. (a cura di), Encyclopedia of Democratic Thought, op. cit., pp. 175-178. Le due obiezioni principali mosse a tale impostazione pertengono all'eccessiva ristrettezza della definizione di democrazia e alla statica visione della politica. 
Robert Dahl definisce incidenter tamtum il consolidamento come la capacità delle istituzioni e della prassi di irrobustirsi «in modo da resistere alla prova del tempo, al conflitto politico e alle crisi»». ${ }^{172}$

Evanescente se non vacuamente tautologico risulta Enrique Baloyra (1987), quando descrive il consolidamento come il processo "whereby installed governments and inaugurated regimes are able to function and avoid or at least recover from deterioration". ${ }^{173}$

Luis Sánchez Agesta ${ }^{174}$ dichiarava che la tappa del consolidamento si caratterizza per la realizzazione normale delle regole del gioco democratico, con consultazioni periodiche dell'elettorato e l'accettazione dei risultati delle urne per la formazione dei governi. Dal punto di vista giuridico avviene la ridefinizione dei ricorsi e delle norme costituzionali in genere; dal punto di vista politico si stabilizza il sistema grazie ad un diritto elettorale. ${ }^{175}$

Staffan Lindberg concentra le proprie analisi sul numero, qualità e frequenza, sul carattere multipartitico delle elezioni e sul fatto fondamentale che i perdenti accettino il responso elettorale, quali efficaci indici di consolidamento: "Transition to and consolidation of democracy are always

Contiguo, ma chiaramente distinto dalla linea di pensiero procedimentale, è l'opera di Whitehead (1989), allorchè individua l'essenza del processo di consolidamento nell'istituzionalizzazione [del nuovo regime]. Ma ciò avviene a mezzo dell'interiorizzazione delle strutture di aperta e competitiva espressione politica.

172 Dahl, R. A., On Democracy, New Haven, 1998; trad. lit. Sulla democrazia, Bari, 2000 , pp. 4.

173 Baloyra, E. A. et al., "Glossary», in: Baloyra E. A., (a cura di), Comparing New Democracies. Transition and Consolidation in Mediterranean Europe and the Southern Cone, Boulder-London, 1987, p. 303. Schedler classifica tale definizione un «largely decorative use of democratic consolidationn: Schedler A., Concepts of Democratic Consolidation, Latin American Studies Association (LASA), Wien-Guadalajara, op. cit., nota 1.

174 Sánchez Agesta, L., "Sobre la consolidación de la democracia en España», in: AA. VV., Sobre la Consolidación de la Democracia [Vornadas sobre consolidación de la democracia, 20-21 settembre, 1985], Buenos Aires, 1987, p. 20.

175 L'obiezione essenziale alla corrente dottrinaria: «una democrazia per dirsi "consolidata" non basta adempia ai requisiti formali di base come elezioni o pluralismo. Si tratta di formulare le condizioni che debbono aversi perchè la democrazia si radichi nello Stato e nella società e divenga improbabile una ricaduta in vecchie strutture autoritarie." Così Fischer-Bollin P., "La consolidación de la democracia en Centroamérica. Retos, obstáculos y perspectivas", in: Contribuciones, vol. 3, 2000, pp. 175-185. 
political struggles of power for sure. These struggles... [are] about the rules of the gamen. ${ }^{136}$

Anche per Jørgen Elklit le elezioni sono un necessario pre-requisito per un consolidamento generale. ${ }^{177}$

Fortemente critico risulta Guillermo O'Donnell: ${ }^{178}$ l'approccio istituzionale, pur portando talora conoscenze utili, quindi in sè buono $\mathrm{e}$ promettente, quantunque deludente ne sia l'applicazione, riceve tutto il suo scetticismo per la vaghezza e l'inclinazione teleologica.

\subsection{La concezione c.d. minimalista}

Con l'etichetta di «modest meaning» 0 «minimalist vieu» alcuni Autori hanno inteso conferire un nucleo di significato minimo al concetto di consolidamento. La loro analisi si dipana lungo l'intreccio tra istituti e sentimenti, anche se la trattazione di una delineazione «minimalista» va ben oltre. La conseguenza — più o meno voluta—è quella di evocare il minimalismo (non politico, ${ }^{179} \mathrm{ma}$ artistico). Si incorre, così, in forme elementari, semplici, essenziali, "quasi geometriche", che delimitano con pochi tratti facilmente percepibili uno spazio preciso. Si tratta di forme razionali più che concettuali. Il punto di frizione sta proprio nella «facilità» di riscontro: entra in gioco la "quotidianità. E prima facie inutile abbarbicarsi ad un range - per quanto circoscritto- di caratteri, per sostenere che questi ultimi sono tratti fondanti una situazione (il consolidamento) che è in divenire. ${ }^{180} \mathrm{Al}$ consolidamento difetta una

176 Lindberg, S. I., A Decent Bouquet or a Putrid Produce?: Democratic Wine in Africa, Dept. of Political Science, paper, Lund University, Lund, 2002.

177 Elklit, J., "Elections: Politics of Polarisation or Inclusion? Inconclusive Reflections Based on Experiences from Three Sub-Saharan African Countries, Lesotho, South Africa, and Tanzania», Arhus, aprile-agosto 2001 ; Bratton M., Second Elections in Africa, in: JoD, vol. 9.3, p. 52.

178 O'Donnell, G., Democratic Theory and Comparative Politics, Wissenschaftszentrum Berlin für Sozialforschung [WZB] Paper 99/004, Berlin, ottobre, 1999.

179 Il minimalismo politico è la tendenza ad impegnarsi al graduale conseguimento di alcuni obiettivi limitati ma ritenuti significativi, di un più ampio programma. Massimalismo politico è atteggiamento intransigente mirato alla realizzazione integrale di un programma, senza mediazioni o compromessi di sorta.

180 «Si suppone che le democrazie mai siano completamente consolidate. Uniche tra i tipi di regime politico, esse possiedono il potenziale di una continua [evoluzione]». Così Schmitter P. C., "Consolidation", in: Lipset S. M. (a cura di), The Encyclopedia of Democracy, I, 1995, pp. 295-299. 
generalizzata quotidianità. ${ }^{181}$ E', come seconda osservazione, difficile individuare un insieme di caratteri tanto ricorrenti in una molteplicità di ordinamenti, da potersi sostenere che proprio essi denotano peculiarmente ciò che è quotidiano, normale, naturale.

Solo leggendo «minimalista» come «minimo» o «minimale» e in chiara contrapposizione alle concezioni che invece vorrebbero includere uno sviluppo estremo (additato come "massimo"), è possibile accettare e comparare filoni d'investigazione così diversi come sono stati i percorsi di Morlino, Di Palma, Linz, Schmitter e Merkel.

\subsubsection{Freezing e conseguimento della legittimazione}

Leonardo Morlino ${ }^{182}$ definisce il Consolidamento democratico come «il processo [composito e variegato] [indotto dal trascorrere del tempo] di congelamento [nei suoi caratteri essenziali] e di adattamento [in quelli secondari] di strutture e norme democratiche [primo consolidamento] che vengono accettate come legittime [secondo consolidamento], in parte o del tutto, dalla società civile». ${ }^{183}$

Date due direzioni del consolidamento, il ruolo degli attori decisionali e la creazione e mantenimento della legittimazione, il riscontro di tratti comuni alle esperienze empiriche di consolidamento democratico ${ }^{184}$ è

181 Diversamente si potrebbe sostenere per una democrazia consolidata. Ci si troverebbe già «dopo» il consolidamento come processo, in una fase susseguente.

182 Morlino, L., "Consolidamento democratico: definizione e modelli, in: Rivista Italiana di Scienza Politica, vol. 16.2, 1986, pp. 197-238; Idem, "Consolidamento democratico: alcune ipotesi esplicativen, ibidem, vol. 16.3, 1986, pp. 439-459. I due saggi si trovano riuniti e in lingua spagnola, con ritocchi minimi, in: Revista Española de Investigaciones Sociológicas, vol. 35, 1986, e in: Pinto J. (a cura di), Ensayos sobre la crisis política argentina, Vol. 3, Biblioteca politica argentina N. 207, Buenos Aires, 1988, pp. 311 377, con il titolo Consolidación democratica. Definición, modelos, hipotesis. Il primo saggio compare in versione inglese come Democratic Consolidation: Definition and Models, in: Pridham G. (a cura di), Transitions to Democracy. Comparative Perspective from Southern Europe, Latin America and Eastern Europe, op. cit., pp. 571-590.

183 Il secondo consolidamento è eventuale: «il processo di progressivo ampliamento della legittimazione del regime" può, in vero, compiersi già durante la instaurazione democratica. Cfr. Morlino L., "Consolidamento democratico: alcune ipotesi esplicative», in: Rivista Italiana di Scienza Politica, vol. 16.3, 1986, p. 439.

184 Morlino indica tra le caratteristiche costanti: a- il ruolo tendenzialmente principale dei partiti e del sistema partitico; 
funzionale alla costruzione di modelli. La ricostruzione di Morlino è volutamente polare, lungo un continuum: ruolo dei partiti-ruolo dei gruppi; tuttavia, mentre le esperienze storiche tendono a catalizzarsi nel primo modello o nei suoi paraggi, negli spazi contigui, il consolidamento attraverso gruppi è solo un'ipotesi irreale.

\begin{tabular}{|c|c|c|c|}
\hline & $\begin{array}{c}\text { CONSOLIDAMENTO } \\
\text { ATTRAVE RSO PARTITI } \\
\text { [M1] }\end{array}$ & $\begin{array}{l}\text { CONSOLIDAMENTO } \\
\text { SIMBIOTICO[M2] }\end{array}$ & $\begin{array}{c}\text { «CONSOLIDAMENTO* } \\
\text { ATTRAVERSO GRUPPI } \\
\text { [M3] }\end{array}$ \\
\hline $\begin{array}{l}\text { RAPPRESENT } \\
\text { AZIONE }\end{array}$ & $\begin{array}{l}\text { il Sistema partitico } \\
\text { controlla la partecipazione }\end{array}$ & $\begin{array}{l}\text { coprotagonismo di partiti e } \\
\text { gruppi }\end{array}$ & $\begin{array}{l}\text { forti gruppi intermedi; } \\
\text { partiti deboli e } \\
\text { disorganizzati }\end{array}$ \\
\hline $\begin{array}{l}\text { PROCESSO- } \\
\text { MECCANISMO }\end{array}$ & $\begin{array}{l}\text { occupazione dello Stato e } \\
\text { dei gruppi }\end{array}$ & $\begin{array}{l}\text { penetrazione dei gruppi } \\
\text { nelle strutture, senza } \\
\text { escludere i partiti }\end{array}$ & $\begin{array}{l}\text { penetrazione dei gruppi a } \\
\text { tutti i livelli Statali }\end{array}$ \\
\hline $\begin{array}{l}\text { PRESUPPOST } \\
\text { O }\end{array}$ & $\begin{array}{l}\text { forte trasformazione } \\
\text { sociale durante } \\
\text { l'autocrazia }\end{array}$ & $\begin{array}{l}\text { esperienza autocratica } \\
\text { sostanzialmente poco } \\
\text { incisiva; forte tradizione } \\
\text { statale }\end{array}$ & $\begin{array}{l}\text { memoria collettiva del } \\
\text { passato [legittimazione } \\
\text { negativa] }\end{array}$ \\
\hline ATTUAZIONE & $\begin{array}{l}\text { varianti per multipli fattori } \\
\text { politici e culturali e storici }\end{array}$ & $\begin{array}{l}\text { meglio si concilia con un } \\
\text { assetto parlamentare (forma } \\
\text { digoverno) }\end{array}$ & $\begin{array}{l}\text { non raggiunge il } \\
\text { Consolidamento ma un } \\
\text { mero mantenimento } \\
\text { instabile del regime } \\
\text { democratico }\end{array}$ \\
\hline
\end{tabular}

Fuori dalla dicotomia "Modello attraverso partiti [M1]- Modello simbiotico[M2]" tertium non datur, con tutta probabilità. Un eventuale "consolidamento carismatico" [M4] sarebbe destinato, secondo l'Autore, a rifluire nel continuum partiti-gruppi.

b- le strutture del regime emergenti quanto a forma di stato, a ruolo del settore economico pubblico e a politiche governative;

c- la messa in opera e mantenimento del compromesso democratico, cioè a dire il ricreare o accrescere la legittimazione democratica;

$\mathrm{d}$-il rispetto della legalità per la capacità del governo di porsi come garante del rispetto delle decisioni assunte ovvero delle leggi;

e- la neutralità o neutralizzazione dei militari;

f- l'assenza di fattori socio-economici: i gruppi imprenditoriali hanno una posizione accidentale verso le istituzioni politiche, mentre le organizzazioni sindacali e contadine vivono per il compromesso democratico. 
Morlino $^{185}$ avrà modo di rielaborare lungo una doppia griglia prospettica il proprio pensiero: una dimensione (consolidamento dall'alto verso il basso) è occupata dalla legittimazione, l'altra (consolidamento dal basso verso l'alto) dall'ancoraggio istituzionale. I quattro ancoraggi tra società e istituzioni riguardano: le organizzazioni partitiche - con un ruolo che può dirsi di "cantoniere»-, i legami relativamente stabili tra interessi e partiti, la diffusione di pratiche clientelari, gli accordi neocorporativi. L'Autore stesso chiarisce che si tratta, in questo caso, di una "teoria locale", spazialmente e temporalmente ben definita (riferita, nella specie, ai quattro Paesi dell'Europa meridionale).

\subsubsection{Crafting e coinvolgimento degli attori}

Anche il consolidamento per Giuseppe Di Palma (1988) ${ }^{186}$ è attività di crafting, utilizzando un'espressione di Linz. ${ }^{187}$ Più precisamente, un'ottica minimalista [per tener distinto quanto il consolidamento è da quanto può comportare], null'altro richiede ai soggetti che la prontezza, la disponibilità a giocare, formando e consolidando la democrazia, in due processi non così diversi. Quindi, una volta intagliate le regole, si tratta di attrarre i giocatori, molti dei quali potrebbero non esser convinti dal gioco o persino potrebbero avversarlo.

185 Morlino, L., Democracy Between Consolidation and Crisis. Parties, Groups and Citizens in Southern Europe, Oxford, 1998 ; Idem, "Consolidamento democratico: La teoria dell'ancoraggion, in: Quaderni di Scienza della Politica, vol. 8.2, 2001, pp. 217247.

Un cenno in: Morlino, L., "Introduzione all'edizione italiana», in: Linz J. J. \& Stepan A., Transizione e consolidamento democratico, op. cit., pp. vii-xvi.

186 Di Palma, G., "Parliaments, consolidation, institutionalization: a minimalist view", in: Liebert U. \& Cotta M., (a cura di), Parliament and Democratic Consolidation in Southern Europe: Greece, Italy, Portugal Spain and Turkey, London-New York, 1990, pp. 31-51, già "Consolidación democrática: una visión minimalista», in: Revista Española de Investigaciones Sociológicas, vol. 42, 1988, pp. 67-92.

187 Linz J. J. e Stepan A., "Political Crafting of Democratic Consolidation or Destruction: European and South American Comparisons", in: Pastor R. A. (a cura di), Democracy in the Americas: Stopping the Pendulum, op. cit., pp. 41-61.

Avviene uno scambio di locuzioni tra Linz e Di Palma: il primo offre il concetto di crafting, il secondo «ricambia» offrendo l'espressione-chiave only game in town. Vedi Linz J. J. \& Stepan A., Problems of Democratic Transition and Consolidation. Southern Europe, South America, and Post-Communist Europe, op. cit., nt. 4, p. 5. 
Di Palma descrive il consolidamento come intaglio delle regole del gioco tanto da prevenire il boicottaggio dello stesso da parte dei giocatori essenziali, in modo tale da rimuovere le potenzialità di caduta permeando di spirito democratico i giocatori e così da mantenere i giocatori nel gioco [«keeping players within the game»].

L'Autore dubita che «la nozione di democratic consolidation [sia] nè più nè meno quella di consolidamento delle istituzioni democratiche, nelle loro strutture interne e relazioni esterne", valea dire il raggiungimento da parte di tali istituti dei caratteri di istituzionalizzazione, coesione, autonomia, autenticità, durevolezza che si presume possano indicare il consolidamento del regime. ${ }^{188}$

Il nucleo essenziale del consolidamento consiste nel definire, fissare e mantenere le regole fondamentali per la competizione politica. Specifica Ulrike Liebert come ciò avvenga in due fasi: una di instaurazione delle regole del gioco e una di congelamento delle stesse. ${ }^{189}$ Inflazionante e parziale è l'utilizzo di concetti quali la legittimazione (quale metro per se del consolidamento), la rotazione dei partiti al governo, la ripetizione e l'accumularsi di elezioni, lo stabilizzarsi dei risultati elettorali, lo sviluppo di una seniority nelle istituzioni elettive. Per l'intuitiva idea che il passare del tempo (e la routinizzazione di eventi, persone e istituti) sia parte del processo di consolidamento. ${ }^{190}$ Risulta allora preferibile riferirsi a substabtive criteria (O'Donnell), che enfatizzino lo sviluppo e lo stabilirsi di specifiche istituzioni democratiche.

$188 \mathrm{Nel}$ lessico dell'Autore, se il consolidamento è visto come un processo limitato nel tempo, spetta al susseguente processo di democratizzazione strutturare e istituzionalizzare le istituzioni democratiche fondamentali.

189 Liebert, U., The Centrality of Parliament in the Consolidation of Democracy: $A$ Theoretical Exploration, ICPS, Working Paper $\mathrm{N}^{\circ} 7$, Barcelona, 1989.

190 Non vale il mero fatto della longevità: una teoria del consolidamento democratico deve essere sensibile alle variazioni di tempo e spazio, deve monitorare le emergenti relazioni tra gruppi di rappresentanza e luoghi di governo. Cfr. Schmitter P. C., "The Consolidation of Political Democracies: Processes, Rhythms, Sequences and Typess, in: Pridham, G. (a cura di), Transitions to Democracy. Comparative Perspectives from Southern Europe, Latin America and Eastern Europe, op. cit., pp. 535-569. 


\subsubsection{La formula the only game in town}

Secondo Linz (1982 ${ }^{191}$ e 1990), ${ }^{192}$ anche in collaborazione con Alfred Stepan, ${ }^{193}$ la democrazia consolidata è una situazione, nella quale la democrazia sia divenuta l'unica alternativa politica esistente $o$, con le parole di Di Palma, "the only game in town".

L'assunto deve validarsi quanto a:

a. comportamento [tratto sociale e politico]. Nessun attore nazionale importante, sia esso istituzionale, politico, sociale o economico, spende risorse significative nel tentativo di raggiungere i propri obiettivi attraverso metodi non democratici. In particolare ciò vale per l'accesso e la conquista del potere politico;

b. atteggiamento [tratto sociale psicologico]. Anche a fronte di crisi politiche ed economiche gravi e gravissime, la maggior parte dei cittadini ritiene che qualsiasi mutamento politico debba compiersi entro il sistema di regole democratiche. In altri termini, la popolazione è convinta che le procedure e le istituzioni democratiche rappresentino il modo più appropriato di governare la vita collettiva, mentre il sostegno per alternative antisistema è limitato o emarginato;

c. Costituzione materiale [tratto istituzionale]. Sia le forze governative che quelle non governative si abituano al fatto che il conflitto politico si risolverà entro un quadro di norme stabilite e che le violazioni inferte a tali norme saranno presumibilmente inefficaci e costose. ${ }^{194}$

191Morlino vede in Linz l'unico precursore, in tempi non sospetri. Morlino cita Linz J. J., "The Consolidation of Regimes: A Theoretical Problem Approach,, VIII World Congress of ISA, Toronto, agosto, 1974; e The Transition from Authoritarian Regimes to Democratic Political Systems and the Problems of Consolidation of Political Democracy, (altrimenti indicato con il titolo Transition to Democracy: A Comparative Perspective), IPSA, Tokyo, marzo, 1982. Il secondo paperè stato poi condensato in Transitions to Democracye pubblicato otto anni più tardi in The Washington Quaterly, vol. 13.3, 1990, pp. 143-164. Cfr., in lingua spagnola, "Transiciones a la democracia", in Revista Española de Investigaciones Sociológicas, vol. 51, 1990.

192 Linz J. J., "Transitions to Democracy», in: The Washington Quaterly, vol. 13.3, 1990, pp. 157-160.

193 Linz J. J. e Stepan A., Problems of Democratic Transition and Consolidation. Southern Europe, South America, and Post-Communist Europe, op. cit..

194 "With consolidation, democracy becomes routinized and deeply internalized in social, institutional, and even psychological life, as well as in calculations for achieving success» in: Linz J. J. \& Stepan A., Problems of Democratic Transition and Consolidation. Southern 
Ripercorrendo lo schema, Richard Gunther, Hans-Jürgen Puhle e Nikiforos Diamandouros ${ }^{195}$ si attestavano su un modello dualistico, cui mancava di esplicitare quando discorsivamente costituiva il terzo polo del ragionamento:

a. comportamento [tratto sociale e politico]. Uno specifico insieme di norme è rispettato e seguito da ogni gruppo politicamente rilevante; la

Europe, South America, and Post-Communist Europe, op. cit., p. 5. In precedenza, "Toward Consolidated Democracies", in: JoD, vol. 7.2, 1996, p. 15, e in: Inoguchi T., Newman E. e Keane J., (a cura di), The changing nature of democracy, Tokyo-New York, 1998 , p. 49.

Alla lettera, gli stessi Autori avevano espresso in termini parzialmente distinti due dei tre contesti:

in termini più ristretri quanto al comportamento [a-]: «Nessun gruppo significativo nel panorama tenta seriamente di rovescire il regime deomocratico o di promuovere violenze interne o internazionali al fine [di implementare cambi in genere o] di secedere. In tali condizioni, il governo eletto emerso dalla transizione non deve curarsi a lungo della questione di come evitare il crollo della democrazian: pare invece giustificare l'allargamento dello spettro degli attori e quello dei fini antidemocratici; in termini più ampi quanto alla Costituzione [c-]: «Ogni attore politico diviene abituato alle regole del gioco democratico", il chè è, francamente, pretendere troppo. Così come deborda Fischer-Bollin (2000) quando rischiede $s u b$ b- l'esistenza di «un massivo appoggio popolare per implementare in situazioni di crisi economica o politica il cambiamento da parte delle istituzioni democratichen: in una democrazia consolidata deve trovar spazio anche la social anorexia, secondo la terminologia di Kostas Vergopoulos [The Political Economy of Democratic Consolidation in Southern Europe, in Ethier, D. (a cura di), Democratic Transition and Consolidation in Southern Europe, Latin America and Southeast Asia, Houndmills-London, 1990, pp. 139-154]. Cfr. Fischer-Bollin P., "La consolidación de la democracia en Centroamérica. Retos, obstáculos $y$ perspectivas", in Contribuciones, vol. 3, 2000, pp. 175-185.

La formulazione embrionale fornita da Linz e Stepan con Gunther nel 1995 poneva come tratto istituzionale un più generico profilo «strutturale»: «Non può esistere dominio riservato di potere se impedisce che importanti politiche pubbliche siano determinate dalla legge, dalle procedure e istituti sanzionati dal nuovo processo democratico" in: Linz J. J., Stepan A. e Gunther R., "Democratic Transition and Consolidation in Southern Europe, with Reflections on Latin America and Eastern Europen, in: Gunther R., Diamandouros N. e Puhle H.-J. (a cura di), The Politics of Democratic Consolidation. Southern Europe in Compatative Perspective, op. cit., pp. 77-123.

195 Gunther R., Puhle H.-J. e Diamandouros N., "Introduction", in: Gunther R., Diamandouros N. e Puhle H.-J. (a cura di), The Politics of Democratic Consolidation. Southern Europe in Compatative Perspective, op. cit., pp. 1-32. Gli stessi Autori chiariscono l'insufficienza ascrittiva delle elezioni e delle istituzioni: Gunther R., Diamandouros P. N. e Puhle H.-J., "O'Donnell's "Illusions": a Rejoinden, in: JoD, vol. 7.4, 1996, pp. 151-159. 
routinizzazione che ne segue agevola l'intuizione di ciò che va a costituire comportamento proprio e la comune accettazione riduce gli eventuali sospetti reciproci;

b. atteggiamento [tratto sociale psicologico]. Le istituzioni democratiche esistenti sono considerate legittime, accettabili e prive di qualsivoglia alternativa legittima: i gruppi significativi ${ }^{196}$ le considerano le uniche strutture legittimate per la contesa politica. Tendenzialmente, il conflitto verrà incanalato per essere materia decisa a mezzo di istituzioni rappresentative, piuttosto che in arene extraparlamentari non regolate e di dubbia rispondenza a canoni democratici;

c. [ancora implicito] Costituzione materiale. La legittimità delle istituzionichiave non è sfidata o le sue regole fondamentali di comportamento politico sono non sistematicamente violate da gruppi politicamente significativi. Comportamento democratico (sub a-) e atteggiamento democratico (sub b-) scoraggiano le élite governanti dal calpestare i diritti dell'opposizione eattenuano i rischi di violenze. Il consolidamento contribuisce poi alla stabilità riducendo l'intensità dell'espressione del conflitto politico e costringendolo in canali istituzionali pacifici.

Il consolidamento - se rapportato all'ondata di democratizzazione (un numero senza precedenti di Paesi ha completato a fine secolo la transizione e sta tentando di consolidare la democrazia) — «richiede molto di più che elezioni e mercati» ${ }^{197}$ (migliorare la qualità della democrazia, mediante l'aumento della soglia economica minima per tutti i cittadini e intensificando la partecipazione politica e sociale). Insomma, non è uno solo il consolidamento, così come non è escluso che le democrazie continuino a progredire: all'interno delle democrazie consolidate esiste una gamma tra democrazie a bassa e ad alta qualità. Non è un ossímoro la caduta di una democrazia consolidata. ${ }^{198}$

196 Un regime può dirsi "sufficientemente consolidato" anche se alcuni suoi cittadini non condividono il consenso democratico o non guardano le sue istituzioni-chiave come legittimate/legittime, fintantochè tali individui o gruppi siano numericamente insignificanti, fondamentalmente isolati dalle forze che sostengono il regime e perciò incapaci di disturbarel sconquassare la stabilità del regime. Così Gunther R., Puhle H.-J. e Diamandouros N., "Introduction", in: Gunther R., Diamandouros N. e Puhle H.-J. (a cura di), The Politics of Democratic Consolidation. Southern Europe in Compatative Perspective, op. cit., p. 8, con riguardo al tema della corrispondenza del singolo ordinamento all'ideal-tipo.

197 Linz e Stepan aprono chiaramente a concezioni massimaliste.

198 Cosi Linz, J. J. e Stepan, A., Problems of Democratic Transition and Consolidation. Southern Europe, South America, and Post-Communist Europe, op. cit., pp. 6-7. 


\subsubsection{La versione ridotta [bipolare]}

Gunther, Puhle e Diamandouros separano in primo luogo il prodotto finale del consolidamento (regime sustainability) dal concetto di consolidamento stesso, incentrato quest' ultimo sul sostegno alle istituzioni rappresentative e sul rispetto per le regole democratiche. ${ }^{199} \mathrm{Di}$ seguito propongono due criteri per rinvenire il consolidamento: la sopravvivenza del regime senza destabilizzazione e l'assenza di rilevanti attori antisistema. ${ }^{200}$

Sull'endiadi di base sistema di istituzioni-persuasione degli attori ${ }^{201}$ concorda Adam Przeworski, in specie quando afferma che la democrazia potrà dirsi consolidata quando l'osservanza, ossia l'agire all'interno del quadro istituzionale, costituisce l'equilibrio delle strategie decentralizzate di tutte le forze rilevanti. ${ }^{202}$ Amalendru Misra definisce il consolidamento come "l'istituzionalizzazione e socializzazione di certi valori e imperativi democratici e la loro susseguente legittimizzazzionem. ${ }^{203} \mathrm{Nel}$ vocabolario indiano, l'istituzionalizzazione fa riferimento alle organizzazioni e procedure del sistema politico, mentre la socializzazione è riferita all'implementazione, coltura e promozione della cultura politica democratica.

Ismael Crespo definisce il consolidamento come «il processo cauto e graduale in cui l'incertezza si istituzionalizza mediante la legittimazione

199 Gunther, R., Puhle, H.-J. e Diamandouros, N., «Introduction», in: Gunther R., Diamandouros N. e Puhle H.-J. (a cura di), The Politics of Democratic Consolidation. Southern Europe in Compatative Perspective, op. cit., p. 13.

200 O'Donnell esce allo scoperto dopo un primo articolo di critica indiretta ed accusa i tre Autori di incoerenza, allorchè non applicano i due criteri a Repubblica Ceca, Ungheria, Polonia e Slovenia, Paesi per i quali parlano di consolidamento quasi-istantaneo. Cfr. O'Donnell G., «Illusions and Conceptual Flaws, in: JoD, vol. 7.4, 1996, pp. 160-168.

201 Specifica Przeworski: «nessuno può immaginarsi di agire al di fuori delle istituzioni democratiche e tutto ciò che gli sconfitti vogliono fare è riprovare [a gareggiare] all'interno delle stesse istituzioni sotto la cui egida hanno perso". Cfr. Przeworski A, Democracy and the Market: Political and Economic Reform in Eastern Europe and Latin America, Cambridge, 1991, p. 26.

202 Meno tecnicamente: «quando nessuno può concepire di agire fuori dalle istituzioni democratiche". Vedi Przeworski A., Democracy and the Market: Political and Economic Reform in Eastern Europe and Latin America, op. cit., p. 29.

203 Misra A., "Democracy and Consolidation: How Consolidated is India's Democracy?", ECPR, XXVII Joint Sessions of Workshops, Workshop 3, Mannheim, marzo 1999. 
delle strutture, delle istituzioni e dei procedimenti democratici da parte degli attori [politicamente rilevanti] con capacità di mediare le domande della società civile». Richiede per il consolidamento un certo grado di partecipazione e di concorrenza politica, vale a dire l'opportunità di dotare di capacità e di meccanismi di equilibrio il sistema politico per risolvere gli ostacoli ereditati dal passato. ${ }^{204} \mathrm{Il}$ tema del consolidamento è l'operatività nella pratica sociale e politica delle regole del gioco democratico e l'ampliamento degli accordi democratici intercorsi tra le élite politiche ad altri strati della cittadinanza.

La costruzione del consolidamento su due dimensioni porta lungo le ascisse l'istituzionalizzazione: gli attori politicamente rilevanti costruiscono un sistema mutuo di aspettative che orientano azioni e relazioni sociali; si tratta del processo di fissazione-congelamento di regole di comportamento politico e del rafforzamento delle strutture e istituzioni dell'autorità $e$ della mediazione. Sulle ordinate è rinvenibile l'inclusività: ${ }^{205}$ il grado di incorporazione nell'arena politica di nuovi attori e temi, per la scomparsa dei limiti che rendono impossibile la apertura dello spazio politico e socio-economico. Il consolidamento ha luogo quando si stabilizzano le due dimensioni in uno spazio accettato come legittimo dalla maggioranza [cos̀ estesa da esser quasi la totalità] della società.

José M. Rosales ${ }^{206}$ riprende l'idea del consolidamento quale processo di riduzione delle incertezze nell'equilibrio di forze che ha reso possibile il cambio di regime e come presidio di riaggiustamento delle identità politiche propiziato dal rafforzamento dell'esperenza democratica interessante tutti gli attori principali. Dalla congruenza tra la revisione ideologica e il carattere democratico che si imprime alle azioni istituzionali

204 Crespo I., "¿Hacia dónde van las democracias latinoamericanas?», in: Alcántara M. \& Crespo I., (a cura di), Los límites de la consolidación democrática en América Latina, op. cit., pp. 13-28.

205 Crespo parla, in vero, di cincertezzan, tacendo (fino alla nota 23) che il carattere è comunque mitigato, per rilevare come l'inclusione di nuovi soggetti sia potenzialmente in grado di mettere in discussione continuamente il sistema. Sistema che dovrebbe connotarsi per tratti flessibili ed elastici. Vedi Crespo I., "¿Hacia dónde van las democracias latinoamericanas?', in: Alcántara, M. e Crespo, I., (a cura di), Los limites de la consolidación democrática en América Latina, op. cit., p. 26.

206 Rosales, J. M., "Tras la consolidación democrática: la normalidad en la articulación entre el sistema de instituciones y una política cívica), in: Alcántara, M. e Crespo, I., (a cura di), Los límites de la consolidación democrática en América Latina, op. cit., pp. 83-101. 
dipenderà la solidità di questo tipo di politica integratice. L'obiettivo di ridurre le incertezze mira a rendere funzionali i conflitti, cioè ad integrarli nel funzionamento dell'ordine democratico, evitando così che la loro comparsa risulti minacciosa per l'equilibrio.

Nel consolidamento ha luogo la normalizzazione [istituzionale o politica]. La transizione ha instaurato istituti e regole legittimate da una ampia maggioranza sociale, tanto che l'ordine democratico - non le sue regole - ha cessato di esser negoziabile.

"La fase di consolidamento può essere intesa come un tempo di apprendistato [politico].» Al consolidamento istituzionale occorre incorporare lo sviluppo di una cittadinanza democratica. Il nuovo ordinamento costituzionale può operare come una piattaforma che faciliti o renda possibile lo sviluppo della cittadinanza come offerta di occasioni di vita, per l'educazione politica e la partecipazione. Occorre civilizzare la politica istituzionale, ampliare gli spazi di espressione per la società civile.

\subsubsection{La versione opposta [assenza di vizi del consolidamento]}

Nello stesso solco di Linz, Samuel Valenzuela ${ }^{207}$ si àncora ad una definizione minimale di democrazia per sostenere che i tradizionali criteri fondanti il consolidamento vanno riqualificati:

- la durevolezza nel tempo è, piuttosto che condizione, un mero attributo del consolidamento;

- non è necessario che sia estinto ogni fattore potenzialmente destabilizzante; ${ }^{208}$

- è probabile, invece, che le istituzioni e/o le relazioni tra soggetti politici debbano esser ridisegnate.

Al contempo, il concetto di consolidamento non esclude processi di rovesciamento della democrazia. ${ }^{209}$ Si ribadisce da parte dell'Autore come nessuna democrazia sarà mai veramente consolidata.

207 Valenzuela J. S., "Democratic Consolidation in Post-Transitional Settings: Notion, Process, and Facilitating Conditions", in: Mainwaring, S., O'Donnell, G. e Valenzuela, S., (a cura di) Issues in Democratic Consolidations: The New South American Democracies in Comparative Perspective, op. cit., pp. 57-104.

208 Il che non significa relativizzare la formula del only game in town.

209 «L'assenza di crisi politiche e di elementi destabilizzanti e la durevolezza di un nuovo ordinamento democratico sono per un verso un test insufficiente e per altro una aspettativa eccessiva per la nozione di consolidamento democratico.» 
Valenzuela fornisce una definizione «in negativo»: poichè è impossibile delimitare una virtual institutionalization senza incorrere in idealizzazioni $\mathrm{o}$, al contrario, eccessivo particolarismo, meglio è ripiegare sull'assenza di fattori ingeneranti una perverse institutionalization.

Identifica le quattro principali deviazioni che inquinano il fine del processo democratico (vale a dire l'autorità di governi democraticamente eletti) e i suoi strumenti (la trasparenza e la centralità del meccanismo elettorale nella formazione del governo):

1. l'esistenza di ambigui e generalizzati tutelary powers: élite non elette risultano sovraordinate a chi riveste cariche a seguito di vittoria elettorale;

2. l'esistenza di reserved domains di autorità e policy-making. si tratta di aree specifiche dell'autorità di governo e della decisione sottratte alla disponibilità o al controllo degli eletti; ${ }^{210}$

2a. l'esistenza di gruppi o settori che godono sistematicamente d'immunità dalla legge; ${ }^{211}$

3. la sottorappresentazione delle forze di minoranza nei processi elettorali;

4. l'idea, diffusa presso qualsivoglia soggetto politico significativo, che le elezioni non siano l'unico modo di stabilire un governo, risultando percorribili mezzi quali il colpo di stato o l'insurrezione.

\subsubsection{La prospettiva degli agenti}

Michael Burton, Richard Gunther e John Higley ${ }^{212}$ definiscono la democrazia consolidata come un idealtipo in cui tutti i criteri procedurali sono soddisfatti e, inoltre, in cui ogni gruppo politicamente rilevante riconosce la legittimità delle istituzioni politiche stabilite e aderisce alle regole democratiche del gioco, come riconosce la filosofia che esso sottende.

210 Valenzuela obietta con l'utilizzo di termini quali «enclavi autoritarie»-che non si allocherebbero al centro della problematica politica del periodo di consolidamento- e «residui» -che non affetterebbero l'ambiente.

211 Whitehead, L., "The Consolidation of Fragile Democracies: A Discussion with Illustrations", in: Pastor R. A. (a cura di), Democracy in the Americas: Stopping the Pendulum, op. cit., p. 81.

212 Burton, M., Gunther, R. e Higley, J., "Introduction: elite transformations and democratic regimen, in: Higley J. e Gunther R. (a cura di), Elites and Democratic Consolidation in Latin America and Southern Europe, Cambridge, 1992, pp. 1-37. 
Il consolidamento può esser pensato come comprensione di particolari caratteri di élite e masse. Per aversi democrazia consolidata è necessario vero consenso delle élite circa il gioco e le istituzioni ed è necessario che le élite nutrano reciproca fiducia e intrattengano relazioni non di poco conto o di poco volume l'una con le altre. Il consolidamento democratico è definito come "élite consensual unity [si badi: gli elementi sono due: il consenso e la rete di interrelazioni tra élite che permette loro di accedere ai canali del potere per tutelare gli interessi di cui sono portatrici] within a fully democratic system". ${ }^{213} \mathrm{Al}$ di là delle etichette utilizzate dagli Autori, ${ }^{214}$ in buona sostanza, la democrazia non è consolidata laddove la partecipazione delle masse non si estenda oltre gli strati benestanti a causa di un suffragio ristretto e/o dove una larga parte della popolazione è costituita da "contadini passivi». In altri termini: dove manchi una sostanziale partecipazione delle masse alle procedure e alle istituzioni democratiche.

\subsubsection{Il consolidamento come metamorfosi dell'incertezza}

Philippe Schimitter s'ingegna ad elaborare un modello euristico il cui fulcro è costituito da una serie di successivi cambiamenti negli istituti e nei comporamenti, a livello di regime e per sotto-livelli. ${ }^{215}$

213 "What principally distinguishes unconsolidated from consolidated democracies is the absence of élite consensual unity» in: Burton, M., Gunther R. e Higley J., "Introduction: elite transformations and democratic regimesn, in: Higley J. e Gunther R. (a cura di), Elites and Democratic Consolidation in Latin America and Southern Europe, op. cit., p. 5. Tanta attenzione alle élite è riservata sul presupposto che esse costituiscano i «deviatori della storia". Cfr. Burton M., Gunther R. e Higley J., "Elites and democratic consolidation in Latin America and Southern Europe: an overvieum, in: Higley J. \& Gunther R. (a cura di), Elites and Democratic Consolidation in Latin America and Southern Europe, op. cit., p. 339.

214 Nella specie, stable limited democracies.

215 Schmitter P. C., "Consolidation", in: Lipset S. M. (a cura di), The Encyclopedia of Democracy, op. cit., pp. 295-299; "The Consolidation of Democracy and Representation of Social Groups", in: American Behavioral Scientist, No 35, 1992, pp. 422-449 ; "The Consolidation of Political Democracies: Processes, Rhythms, Sequences and Types, in: Pridham G. (a cura di), Transitions to Democracy. Comparative Perspectives from Southern Europe, Latin America and Eastern Europe, op. cit., pp. 535-569; "Dangers and Dilemmas of Democracy", in: Diamond L., Plattner M. F. (a cura di), The Global Resurgence of Democracy, Baltimore-London, 2a ed., 1996; già in: JoD, vol. 5.2, 1994, pp. 57-74; 
Il consolidamento di un regime consiste nel trasformare in strutture gli arrangiamenti accidentali e le soluzioni contingenti $\mathrm{i}^{216}$ che emergono durante gli incerti scontri della transizione, cioè a dire in relazioni (di cooperazione e di competizione) che sono riconosciute, note, regolarmente praticate e abitualmente e volontariamente accettate. ${ }^{217}$

Il consolidamento di un regime democratico consiste nel convertire le soluzioni ad hoc in strutture stabili di accesso, inclusione, assunzione delle decisioni e gestione delle risorse, lungo il principio base della partecipazione.

Nelle sue riflessioni sulle "trappole ed errori della consolidologia", l'Autore ${ }^{218}$ dichiara: "Non è la democrazia ciò che si sta consolidando, ma uno o altro tipo di democrazia". Ogni tipo di democrazia ha una distinta modalità di consolidarsi, specie quanto a ritmo e cadenza; nessun

"Transitology: The Sciences or the Art of Democratization?", in: Tulchin J. e Romero B. (a cura di), The Consolidation of Democracy in Latin America, op. cit., pp. 11-41; "Some basic assumptions about the consolidation of dernocracys, in: Inoguchi T., Newman E. e Keane J., (a cura di), The changing nature of democracy, op. cit., pp. 23-36.

Cfr. Schmitter P. C. e Karl T. L., "What Democracy Is... and Is Not”, in: Diamond L., Plattner M. F. (a cura di), The Global Resurgence of Democracy, Baltimore-London, $2^{\text {a }} \mathrm{ed}, 1996$; già in: JoD, vol. 2.3, 1991, pp. 75-88 e in: Pridham G. (a cura di), Transitions to Democracy. Comparative Perspectives from Southern Europe, Latin America and Eastern Europe, op. cit., pp. 3-16; Karl T. L. e Schmitter P. C., "Modes of Transition in Latin America, Southern and Eastern Europen, in: International Social Science Journal vol. 128, 1991, pp. 269-284 ; Schmitter P. C. e Karl T. L., "The Conceptual Travels of Transitologists and Considologists: How Far to the East Should They Attempt to Go.», in: Slavic Review, vol. 53, 1994, pp. 173-185 ; Schmitter P. C. e Santiso J., "Three Temporal Dimensions to the Consolidation of Democracy", in: International Political Science Review, vol. 19.1, 1998, pp. 69-92.

Ma già un accenno in O'Donnell G. e Schmitter P. C., "Negociación (y renegociación) de pactos", in O'Donnell G., Schmitter P. C., Whitehead L., Transiciones desde un gobierno autoritario, versione in castigliano curata da Oszlak O., IV, Conclusiones Tentativas sobre las Democracias Inciertas, Barcelona-Buenos Aires, 1988 (1994), cap. 4. Già Transitions from Authoritarian Rule, Tomo IV, Tentative Conclusions about Uncertain Democracies, Baltimore-London, 1986.

216 Il radicamento di ciò che inizialmente sono interazioni fortuite, arrangiamenti episodici, soluzioni ad hoc, patti temporanei, momentanee alleanze, progetti accidentali, norme prudenziali.

217 L'idea base è che le relazioni sociali possano diventare strutture sociali (autonome nel loro funzionamento interno e resistenti ai cambiamenti indotti dall'esterno).

218 Schmitter P. C., "Transitology: The Science or the Art of Democratization?", in Tulchin J. S. e Romero B. (a cura di), The Consolidation of Democracy in Latin America, op. cit., pp. 11-41. 
specifico percorso verso il consolidamento è una garanzia per la futura stabilità.

Ancora tre le dimensioni, pur se non esplicitate:

1. procedimentale. Nel consolidamento la regola fondamentale è la competizione nella formazione del governo e la contingenza nel mobilitare il consenso. Vengono allora suggellate competizioni regolari e incerte nei risultati in un sistema di regole e arene politiche. Il minimum procedurale importa il diritto di associazione e di contestazione, il diritto di voto e il rule of law. ${ }^{219}$

2. strutturale. Il minimum strutturale conseguente al principio della competizione politica comporta pluripartitismo, riconoscimento dei gruppi, responsabilità dell'Esecutivo. Fondamentali sono due poli: l'arrangiamento del sistema partitico e l'assicurarsi la non fuoriuscita dei partiti sconfitti. ${ }^{220}$ Viene poi l'aspetto istituzionale: "Il cuore del dilemmaconsolidamento risiede nel venir fuori con un set di istituzioni che i politici possono concordare e che ai cittadini è richiesto supportare.» Ancora due poli: la scelta degli istituti e la formazione di uno stratum politico.

3. attitudinale. Gli attori aggiustano le proprie aspettative al radicamento delle soluzioni che in nuce si andavano delineando durante la transizione, arrivando a stimare le regole e risorse di tali strutture emergenti come accettabili se non desiderabili. ${ }^{221} \mathrm{La}$ risorsa principale del consolidamento dovrebbe essere la cittadinanza. I valori di procedure e strutture debbono ottenere legittimazione. In capo alle persone e alle collettività (vale a dire politici e cittadini) è richiesta la conoscenza, la volontaria accettazione e la regolare pratica delle relazioni democratiche. ${ }^{222}$

219 Il minimo indispensabile indicato nel $1971 \mathrm{da}$ Dahl necessita poi di ulteriori complesse relazioni.

220 Schmitter P. C., "The Consolidation of Political Democracies: Processes, Rhythms, Sequences and Typen, in: Pridham G. (a cura di), Transitions to Democracy. Comparative Perspectives from Southern Europe, Latin America and Eastern Europe, op. cit., pp. 535-569.

221 Nelle moderne democrazie stabili, alti livelli di identificazione, approvazione etnica ed entusiasmo partecipativo sono raramente la norma, sono anzi visti con scetticismo e scoraggiati. Tali valori possono essser importanti nella transizione (the popular upsurge), ma nel lungo corso ciò che importa (ed è sufficiente) è che la cittadinanza abbia un diffuso senso di naturalità e appropriatezza per il regime, anche se con desencanto.

222 Schmitter P. C., "The Consolidation of Democracy and Representation of Social Groups", in: American Behavioral Scientist, No 35, 1992, pp. 422-449. 
Va detto che Schmitter si guarda bene dall'incentrare il consolidamento sulla capacità di scelta e l'azione volontaristica. Arrivare ad una stabile soluzione non è cosa facile. Le scelte sono intrinsecamente conflittuali e sono estrinsecamente consequenziali. In una certa misura tali scelte sono materie sostanziali anticipate dagli attori e incorporate nel compomesso che essi stipulano con riguardo alle procedure. Ma residuano molti spazi e margini per errori e conseguenze non volute.

Nel breve termine, il consolidamento dipende dalla capacità di agenti politici e cittadini di uscire con una soluzione ai conflitti intrinseci sulle regole. Nel lungo periodo, dipenderà, invece, dall'impatto estrinseco che le politiche elaborate sotto l'egida di tali regole avrà sui gruppi sociali. ${ }^{223}$

A consolidamento avvenuto, la democrazia avrà incertezza istituzionalizzata circa ruoli e policy areas, ma avrà rassicurato i propri cittadini che la competizione per occupare uffici o esercitare influenze sarà trasparente e circoscritta a un previsibile range di risultati.

Le democrazie moderne si collocano su questa "bounded uncertainty" e il «contingent consent» degli attori che rispettano i risultati che esse producono. ${ }^{224}$

\subsubsection{La concezione istituzionale avanzata}

Wolfgang Merkel (1995) ${ }^{225}$ riscontra ${ }^{226}$ nel contesto dell'Europa centro-orientale la simultanea trasformazione dei sistemi economico, sociale e politico ["an apparent trilemma», ovvero il «magical triangle» di Claus Offe].

223 Schmitter P. C., Intermediaries in the Consolidation of neo-Democracies: The Role of Parties, Associations and Movements, Universitat Autónoma de Barcelona, Institut de Ciències Polítiques i Socials [ICPS], Working Paper No 130, 1997. Tuttavia va ricordato che "some time must elapse before the new rules of cooperation and competition produce observable results".

224 Karl, T. L. e Schmitter, P. C., "Modes of Transition in Latin America, Southern and Eastern Europe", in: International Social Science Journal, vol. 128, 1991, pp. 269-284.

225 Merkel, W., Institutions and Democratic Consolidation in East Central Europe, Instituto Juan March, Centro de Estudios Avanzados en Ciencias Sociales [CEACS], Working Paper No 86, dicembre 1996.

226 Nello studio del cambio politico (ed in particolare della transizione), in aperto contrasto agli approcci «economy first» e «civil society first", l'Autore propugna la tesi del "polity and politics first". 
Solo le istituzioni politiche giocano, in realtà, un ruolo primaziale, in quanto, fungendo da connettore tra il livello macro del sistema e il livello micro dell'azione politica, sviluppano una certa configurazione di poteri e incoraggiano precisi interessi, attori e comportamenti. ${ }^{227}$

Il processo di consolidamento viene tratteggiato mediante tre fasi concatenate:

1. consolidamento strutturale, che attiene alla Costituzione e alle istituzioni politiche;

2. consolidamento rappresentativo, che riguarda le organizzazioni intermedie di interessi, i partiti e i gruppi d'interesse;

3.- consolidamento ideologico, che descrive il comportamento dei cittadini, nella direzione di un sostegno preciso e diffuso, dell'emersione di una vivida società civile.

A ben vedere il completamento della sola prima di tali fasi è propedeutico alle successive, ben potendo la seconda e la terza iniziarsi in costanza della precedente, ma non potendo compiersi salvo a avvenuto consolidamento della prima. E' per questo motivo che le istituzioni ${ }^{228}$ - e la Costituzione in particolare- determinerebbero «to a very high degree the consolidation prospects of young democracies».

La Costituzione dunque deve godere di legittimazione formale e di legittimazione empirica. La legittimazione formale arriva dall'alto, mediante il legale instaurarsi del potere costituente; dall'interno, per i metodi della stessa assemblea costituente; e dal basso, qualora la Carta sia ratificata dal popolo. La legittimazione per così dire empirica deriva dalla risoluzione

$227 \mathrm{E}^{\prime}$ il limite dichiarato della "polity and politics first" theory: le istituzioni affettano positivamente o negativamente l'ambiente sociale e quello economico, ma tali ambienti, a loro volta, costituiscono chiari pre-requisiti per aversi istituzioni regolarmente funzionanti.

228 Merkel richiama quattro linee di lettura per le istituzioni:

a- sociologica: la macro-strutturale tradizione di trasformazione del regime che poggia sulle classi sociali e lo Stato (Moore; Rueschemeyer, Huber Stephens e Stephens);

b- politica: la lettura istituzionale tradizionale (Lijphart, Linz, Diamond);

c- organizzazionale/organizzativa: il neo-istituzionalismo, che ricerca come le istituzioni influenzino il comportamento strategico degli attori politici o come le istituzioni stesse possano esser forgiate in ordine allo stimolare comportamenti cooperativi (March e Olsen; Ostrom);

d- storica: l'istituzionalismo storico che vede il "capitale sociale» come un pre-requisito socio-economico per l'efficienza istituzionale (Putnam).

Occorre, secondo Merkel, combinare tutti e quattro gli approcci per far affiorare il mesolivello altrimenti negletto del usystem change». 
della triplice tensione [esistente tra conflict vs. consensus, representation vs. governability, consent vs. efficiency ${ }^{229}$ non in favore di un polo d'alternativa, ma piuttosto dall'amalgama e contrattazione che origina le istituzioni democratiche. Istituzioni e procedure che devono poi sopravvivere abbastanza affinchè norme e valori della democrazia siano sufficientemente interiorizzati e diventino abitudine.

Per condurre al successo il consolidamento, le istituzioni politiche debbono avere due tratti:

- essere quanto inclusive possibile;

- capitalizzare un adeguato potenziale risolutivo in vista di conflitti sociali e politici. In tal senso andrebbe evitata la polarizzazione e assicurato un grado sufficiente di accettazione sociale senza minare la stabilità delle istituzioni stesse, mentre l'efficenza dovrebbe rendere possibili decisioni appropriate.

Caroline Boussard (2000) richiede un cambio di ottica, affinchè si presti attenzione alle istituzioni e procedure informali. Su tutte, la società civile, non per se, ma come fattore di cambio, secondo tre linee fondamentali: per lo sviluppo di una cultura politica democratica, per il rafforzamento della società politica e per la riduzione della povertà.

Slittare dalle istituzioni formali a quelle informali consentirebbe di mutare approccio teoretico: non più spiegazioni mediante «simple linear model[s]》, bensì la lettura del consolidamento come «constantly ongoing process, nè lineare, nè irreversibile. ${ }^{230}$

\section{4 $\mathrm{La}$ concezione istituzionale-massimalista}

Di fondo sta il rifiuto dell' 'idea di democrazia elettorale o, quantomeno, dell'idea che la democrazia elettorale sia un [appagante] punto d'attracco.

Per un certo filone di pensiero [ad esempio Dahl] ${ }^{231}$ il consolidamento, che comprende la dimensione procedurale senza tuttavia a questa limitarsi, implica il raggiungimento di un elevato grado di istituzionalizzazione delle

229 Per un'ampia trattazione delle tre tensioni, Diamond L., "Three paradoxes of Democracy", in: JoD, vol. 1.3, 1990; successivamente in: Diamond L. e Plattner M. F., (a cura di), The Global Resurgence of Democracy, op. cit., pp. 95-107.

230 Boussard, C., Civil Society in the Consolidation Process. Illustrations from Central America, op. cit..

231 Dahl, R., Sulla Democrazia, op. cit.. 
organizzazioni democratiche complesse, quali i poteri esecutivo, legislativo e giudiziario e il sistema dei partiti. ${ }^{232}$

Più ristretta al campo procedimentale, la visione di chi sostiene che per aversi consolidamento sia necessaria l'alternanza al potere ${ }^{233}$ (Huntington), pur in ottica «minimalista» quanto a democrazia, ${ }^{234}$ richiede addirittura un "doppio ricambio». ${ }^{235}$

Correttamente Schmitter ${ }^{236}$ si riferisce alla rotazione (o maggior riallineamento dei partiti al potere) come mero indicatore non del consolidamento, quanto di una successiva fase di persistenza della democrazia.

George Sørensen ${ }^{237}$ indica la «definizione più esigente» di consolidamento quella indicante uno status non raggiunto fino a che tutte le istituzioni democratiche non siano state forgiate e la nuova democrazia si sia dimostrata capace di far fronte alle sfide più difficili, [e] capace di trasferire il potere ad un partito già di opposizione.

232 Obietta Linz: nessuna democrazia sarà mai "consolidata» in una simile accezione. Vedi "Transitions to Democracy», in: The Washington Quaterly, vol. 13.3, 1990, p. 158.

233 Przeworski, A., Alvarez, M., Cheibub, J. A., Limongi, F., «What makes Democracies Endure?", in: JoD, vol. 7.1, 1996, pp. 39-55.

234 «Le democrazie si consolidano quando le persone imparano che il sistema democratico risolve il problema della tirannide, non tutti gli altri». «Democrazia non significa risolvere i problemi, ma poter rimuovere i governanti [e l'essenza del comportamento democratico sta nel compiere la seconda azione proprio perché è impossibile ottenere la prima]». Cfr. Huntington S. P., The Third Wave. Democratization in the Late Twentieth Century, Norman, 1993, pp. 266-267 (trad. it. La terza ondata. I processi di democratizzazione alla fine del XX secolo, Bologna, 1995, p. 281).

235 Huntington, S. P., The Third Wave. Democratization in the Late Twentieth Century, op. cit., p. 285. Il «two turnoven è richiesto sottoforma di test [per l'istituzionalizzazione di lungo corso].

236 Schmitter P. C., "The Consolidation of Political Democracies: Processes, Rhythms, Sequences and Types", in: Pridham G. (a cura di), Transitions to Democracy. Comparative Perspectives from Southern Europe, Latin America and Eastern Europe, op. cit., pp. 535-569.

237 Sørensen G., Democracy and Democratization. Processes and Prospects in a Changing World, Boulder, 1993, p. 45. Considerato che nessuna democrazia risulterebbe consolidata, tanta è la pretesa di una simile concezione, l'Autore aderisce alla definizione di Linz. 


\subsection{La concezione massimalista}

\subsubsection{Il primo stadio: politico}

Diamond ${ }^{238}$ segnala una serie di cambi [oltre a quelli istituzionali, di politiche e di atteggiamento-comportamento] che avrebbero l'effetto di «istigare» il consolidamento.

A. Cambio di cultura politica [costituzionale]. Il consolidamento implica ben più di un accordo sulle regole per disputarsi il potere: implica restrizioni fondamentali e autoimponibili all'esercizio del potere stesso, che a loro volta richiedono un patto tra le élite. Solo quando tale impegno è fortemente credibile, un governante sviluppa un interesse proprio all'adesione alle regole del gioco, il che fa sì che tali regole costituzionali si impongano da sè medesime.

B. Cambio normativo conseguente. "Per sopravvivere, una Costituzione deve avere molto più di un attrattivo filosofico o teorico: la maggiornaza dei cittadini deve considerarla degna di esser difesa" (Weingast). ${ }^{239}$

Diamond indica poi riforme e rafforzamenti istituzionali:

a. Molti di essi migliorano la governabilità: fortificano la capacità dello Stato, liberalizzano e razionalizzano le strutture economiche, garantiscono l'ordine sociale e politico mantenendo le libertà fondamentali, migliorano la responsabilità orizzontale e l'imperio della legge, controllano la corruzione;

b. Altri perfezionano le funzioni rappresentative del governo democratico: rafforzano i partiti politici e il loro vincolo ai gruppi sociali, riducono la frammentazione del sistema partitico, rafforzano la capacità autonoma e la responsabilità pubblica di legislativi ed esecutivi locali, vigorizzano la società civile;

c. Particolari sono i provvedimenti tesi a ridurre l'influenza militare in questioni non militari, o a promuovere la convivenza tra differenti gruppi etnici e nazionali.

Sørensen è travisato sul fondamentale punto da Centellas M., The Consolidation of Polyarchy in Bolivia, 1985-1997, Kalamazoo-Chicago, 1999.

238 Diamond, L., Is the Third Wave of Democratization over? The Imperative of Consolidation, op. cit..

239 Weingast, B. R., The Political Foundations of Democracy and the Rule of Law, Stanford, 1996. 


\subsubsection{Il secondo stadio: sociale}

Philippe Schmitter indica quale "risultato normativo» del proprio modello la probabilità di ulteriore democratizzazione: occorre valutare se il consolidamento si sia compiuto così da precludere ulteriori estensioni del principio di cittadinanza o se esso aumenti la probabilità che l'uguaglianza di diritti, obblighi e condizioni venga espansa per coprire nuove arene, tematiche e istituzioni, specie nel sociale e nel campo economicoIn altri termini: sarà capace la nuova Democrazia di rimuovere o diminuire le diseguaglianze? «In tal senso il consolidamento è l'inizio e non la fine delle politiche.. ${ }^{240}$

Dharragh Hunt ${ }^{241}$ definisce in prima battuta il consolidamento (quello che la prima letteratura chiamava «socialization») l'estensione della democrazia a più istituzioni e da diritti formalmente sanzionati a diritti sostanziali.

In generale, l'approccio massimalista guarda a partiti, gruppi d'interesse, movimenti d'opinione; a che vi siano mass media più informativi, una cultura politica del compromesso e della negoziazione, concertazione tra opposti interessi organizzati, rispetto per i diritti individuali; ai caratteri delle organizzazioni e istituzioni democratiche. In particolare, in quest'ultima accezione, Gianfranco Pasquino riferisce il processo di democratizzazione ad un'evoluzione interna a tutte le strutture operanti in un regime democratico: «il massimo di democrazia [si consegue e mantiene] una volta che tutte le strutture, tutte le organizzazioni e tutte le associazioni operanti in un regime democratico [siano] state democratizzates. ${ }^{242}$

Dahl prospetta un processo di democratizzazione della vita della comunità all'interno dello Stato-nazione. ${ }^{243}$ Gerald Hyman richiede che la democrazia sia «l'unica regola» non solo a livello di procedure di governo,

240 Schmitter P. C., "The Consolidation of Political Democracies: Processes, Rhythms, Sequences and Types", in: Pridham G. (a cura di), Transitions to Democracy. Comparative Perspectives from Southern Europe, Latin America and Eastern Europe, op. cit., pp. 535-569.

241 Hunt, D., Transitology and Political Change in Russia. Russian and East European Politics, Helsinki, 2001.

242 Pasquino, G., La democrazia esigente, Bologna, 1997, p. 18.

243 Dahl, R. A., Democracy and Its Critics, New Haven, 1989 (trad. it. La democrazia e i suoi critici, 2a ed., Roma, 1997). 
ma nella vita pubblica in genere, nella vita sociale ad ogni livello, nelle strutture di gruppo, nelle organizzazioni non governative, nelle strutture d'intermediazione sociale, «dai gruppi di gioco dei bimbi, ai club sportivi, alle associazioni di quartiere, al governo". Deve diventare "secondo natura" che quando i gruppi ad ogni livello necessitano di governarsi, applichino le procedure democratiche. ${ }^{244}$ Boussard va oltre la propria ricostruzione, quando richiede la democratizzazione delle strutture interne delle unità della società civile e, implicitamente, dei legami tra società civile e società politica. ${ }^{245}$

Diamond rileva: la maggioranza delle democrazie della terza ondata non si è consolidata e nessuna democrazia elettorale non liberale ha raggiunto il consolidamento: ${ }^{246}$ conferma empirica a una sfiducia ontologica, per incompletezza presunta.

Nella concezione evolutiva della democrazia, il consolidamento risulta precluso o disintegrato causalmente dagli effetti della superficialità $e$ decadenza istituzionale. Quanto meno rispettosi dei diritti e delle libertà sono i comportamenti dei governanti, tanto più debole sarà il consenso procedimentale che imbastirà la democrazia, risultando gioco forza ostacolato il consolidamento. Quanto più superficiale, escludente, irresponsabile e violatore dei diritti sarà il regime elettorale, tanto più difficile sarà guadagnare o conservare una legittimità profonda a livello di masse. ${ }^{247}$ Dunque, per potersi consolidare, le democrazie elettorali debbono approfondirsi e farsi più liberali. Ciò esige una maggior responsabilità dell'Esecutivo di fronte alla legge e il controllo di altri poteri e della cittadinanza; la riduzione degli ostacoli alla partecipazione e mobilitazione politica dei gruppi marginalizzati; una protezione più efficace dei diritti politici e civili di tutti i cittadini.

244 Gerald F. Hyman G. F. (et. al.), Conducting a Democracy and Governance [DG] Assessment: A Framework for Strategy Development, Center for Democracy and Governance, U.S. Agency for International Development [USAID], Development Experience Clearinghouse [DEC], Document PN-ACG-505, Washington DC, dicembre 1999.

245 Boussard, C., Civil Society in the Consolidation Process. Illustrations from Central America, op. cit.

246 Diamond, L., "El final de la tercera ola y el futuro global de la Democracia", in: López E., Mainwaring S. (a cura di), Democracia: discusiones y nuevas aproximaciones, Buenos Aires, 2000, pp. 13-102.

247 E i golpisti vedranno di minor costo il rovesciamento del sistema democratico. 
Secondo Diamond, tre processi conducono al consolidamento:

1. l'approfondimento (di cui sopra);

2. l'istituzionalizzazione, vale a dire l'inclinazione a favore di regole routinarie, ricorrenti e prevedibili di comporamento politico. Ciò implica una forte convergenza e un' interiorizzazione di regole e procedimenti comuni di concorso e azione politici;

3. la performance politica del regime. Il regime deve produrre risultati politici sufficientemente positivi, anche se non immediati, tanto da costruire un'ampia legittimità politica o quantomeno per evitare la cristallizzazione di sacche di resistenza alla legittimità.

Se Diamond rileva come la maggioranza delle nuove democrazie lungo il pianeta non si sia consolidata, ${ }^{248}$ Joe Faweraker obietta, in linea teoretica, che «le democrazie si consolideranno se diventeranno sufficientemente liberali», per poi constatare amaramente il consolidamento delle illiberal democracies. ${ }^{249}$

Quanto ad autori che debordano da una trattazione invece condotta in massima parte entro $\mathrm{i}$ confini del c.d. consolidamento in senso minimalista, sembra opportuno riportare due esempi, agli estremi del decennio degli anni novanta.

Alejandro Moreno Menéndez descrive il consolidamento in termini di problemi tipici. Nel dettaglio ne indica quattro: l'ingegneria politica, la neutralizzazione delle élite autoritarie, l'accettazione da parte di larga parte di masse ed élite della democrazia come «the only game in town», l'implementazione di riforme economiche per stabilizzare situazioni di crisi. Le fragilità del costrutto sono evidenti nella imprecisione di ciascuno dei quattro enunciati. ${ }^{250}$

248 Diamond, L., "Future of Democracy", in: Clarke P. B. e Foweraker J. (a cura di), Encyclopedia of Democratic Thought, op. cit., pp. 154-161.

249 Le democrazie possono dirsi consolidate senza essere liberali, e possono essere liberali senza esser consolidate. Non è il rule of law a consolidare o esser consolidato, bensì le regole informali che si fondono nel clientelismo e nel patrimonialismo. «Il risultato non è il rule of law, ma the rule of informal rules che favorisce il più forte e sistematicamente discrimina il più debole». In tale ottica, le democrazie della terza ondata potranno pure consolidarsi, ma ciò che si sarà consolidato sarà ben diverso/differente dal modello di democrazia liberale che le ha precedute. Cfr. Foweraker J., "Waves of Democracy", in: Clarke, P. B. e Foweraker J. (a cura di), Encyclopedia of Democratic Thought, op. cit., pp. 705-709.

250 Menéndez Moreno, A., Political Cleavages. Issues, Parties and the Consolidation of Democracy, Boulder-Oxford, 1999, p. 19. 
Sono sei, invece, i principi e le categorie di trasformazione ad essere riconosciuti da Diane Ethier come favorevoli al consolidamento delle poliarchie: ${ }^{251}$ la libera espressione di interessi divergenti, dovuta all'autonomizzazione della società civile e alla conseguente creazione di enti esponenziali intermedi; la formazione di governi di maggioranza e la pacifica alternanza al potere, dovuta allo sviluppo di partiti, legittimati, in senso pluralista; l'accrescimento della legittimità di organizzazioni appartenenti alla società civile e dell'efficienza della rapprensentazione politica degli interessi, dovuta all'approfondirsi delle relazioni tra partiti politici e gruppi d'interesse; l'accettazione della Costituzione da parte della maggioranza dei cittadini, gruppi e partiti; la fondazione di meccanismi di consultazione ("concertazione») tra Stato, partiti e principali gruppi d'interesse (sindacati e associazioni imprenditoriali); l'approfondimento della dimensione sostanziale del principio di cittadinanza.

Jacqueline Jiménez individua due percorsi dottrinali che hanno cercato di coniugare la teoria della modernizzazione con quella del cambio politico, per allocare il grado di sviluppo socio-economico, il peso della classe media, il livello di comunicazione, urbanizzazione e educazione. ${ }^{252}$

A) Un primo filone, piuttosto che correlare i requisiti della democrazia alla Transizione (è il caso di Lipset), propugna il riconoscimento dell'esistenza di relazioni sistematiche tra le caratteristiche dei regimi politici e i modelli di sviluppo nazionale, l'analisi degli effetti della democrazia politica sulla crescita economica e sulla eguaglianza socio-economica.

B) Un secondo filone preferisce distrarre i requisiti dalla democrazia per riferirli al consolidamento [o alla stabilità]. Opportuno sarebbe, allora, correlare fattori storici di larga portata (quali un deficit democratico cronico o esperienze previe di pluralismo politico) con la capacità di consenso, $o$ le strategie di negoziazione delle élite, i meccanismi politici, le condizioni socio-economiche e la capacità dei partiti a maneggiare la crisi economica e favorire la stabilità della democrazia.

251 Ethier, D., "Introduction: Processes of Transition and Democratic Consolidation: Theoretical Indicators", in: Ethier, D. (a cura di), Democratic Transition and Consolidation in Southern Europe, Latin America and Southeast Asia, op. cit., pp. 3-21.

252 Jiménez, J., "Los partidos politicos en el nuevo marco institucional. Presentación", in: Alcántara, M. \& Crespo I., (a cura di), Los límites de la consolidación democrática en América Latina, op. cit., pp. 129-131. 
La novità consisterebbe nel considerare quelli che erano requisiti per l'esistenza della democrazia ${ }^{253}$ (variabili dipendenti) come frutti della democrazia (variabili indipendenti), il tentativo di sviluppare un'ottica interattiva che correlazioni i limiti strutturali con la formazione di opzioni contingenti. In quest'ottica opera Terry Lynn Karl: gli ampi cambi strutturali possono essere altamente favorevoli alla democratizzazione: si incorporano alle istituzioni e regole politiche che nel consolidamento modellano le preferenze e capacità degli individui.

\subsubsection{La letteratura latinoamericana}

«La visione della democrazia in America Latina è stata vincolata alla giustizia sociale, il che ha stornato l'attenzione dal sistema politico in favore del tema delle opportunità di partecipazione sociale e economica»: è la sintesi efficace di Ricardo Córdova e Günther Maihold. ${ }^{254}$

René Antonio Mayorga riassume nei suoi scritti le linee di fondo che portano gli studiosi latinoamericani ad abbracciare concezioni sempre più vaste di consolidamento: il consolidamento "dipende dalla capacità dei governi democratici di affrontare le maggiori sfide [usailing against the wind"] al raggiungimento di una miscela ragionevolmente efficacie di democrazia, economia di mercato ed equità [socio-economica]». ${ }^{255}$

Per questo finiscono col concorrere a determinare il significato di consolidación democrática:

- il ruolo strategico dei partiti politici quali istituzioni rappresentanti e canalizzanti interessi sociali;

- l'accettazione dei risultati elettorali e l'emergere del Parlamento come sorgente fondamentale della legittimità;

- l'assenza o irrilevanza di forze politiche antisistema;

- la subordinazione dei militari al potere civile;

- l'alternanza al potere;

- il tratteggio di strategie di riforma istituzionale dello Stato e del sistema politico;

253 Approccio causale sostenuto dai funzionalisti.

254 Córdova, R. e Maihold, G., Democracia y ciudadanía en Centroamérica. Perspectivas hacia el 2020, Institut für Iberoamerika-Kunde, Hamburg, 2000.

255 Mayorga, R. A., Parliament arized Presidentialism, Moderate Multiparty System and State Transformation: The Case of Bolivia, Centro Boliviano de Estudios Multidisciplinarios (CEBEM), setiembre, 1996. 
- la stabilità economica con moderati tassi di crescita;

- l'emergere di un "triplice consenso - procedurale, normativo e sostanziale $\longrightarrow$ sulle procedure e le regole del gioco, sulla modernizzazione e sulle fondamentali guidelines in tema di politica economica.

Miguel Centellas, ${ }^{256}$ partendo dalla considerazione di Dahl di "democracy as a moving target", obiettivo che mai è raggiunto, ${ }^{257}$ definisce una poliarchia consolidata come «un regime in cui la democrazia politica è istituita anche mentre il regime fa espliciti sforzi (e non meri atteggiamenti o retorica) verso una più profonda democratizzazione della società politica, sociale o economica. Un regime può configurarsi quale poliarchia consolidata pur se la democrazia sociale o economica manca ma - si sottolinea - temporaneamente».

\subsection{La concezione socio-economica}

Non è sufficiente conservare ["difendere»] la democrazia [a mezzo degli strumenti dello "stato di diritto" e della "cultura politica"]: occorre raggiungere condizioni economiche, sociali e politiche che permettano di alimentare l'ordine democratico. Secondo Christoph Müller, ${ }^{258}$ il consolidamento della democrazia dipende dalla prospettiva di creare una situazione che produca e ripruduca la democrazia in maniera continua. Il "modello di riproduzione" ${ }^{259} \mathrm{e}$, appunto, un sistema che permette la ripetizione di processi sociali essenziali, specialmente economici, e di garantirli nel lungo periodo, di modo che assicurino il funzionamento sistemico permanente. La domanda di partenza è propriamente: quanto

256 Centellas, M., The Consolidation of Polyarchy in Bolivia, 1985-1997, KalamazooChicago, 1999.

257 Dahl, R. A., Democracy and Its Critics, op. cit.

258 Müller, C., "Consolidación de la democracia. Viejos problemas y nuevas experiencias desde una perspectiva alemana", in: AA. VV., "Sobre la Consolidación de la Democracia" [Jornadas sobre consolidación de la democracia, 20-21 settembre 1985], Buenos Aires, 1987, pp. 37-55.

259 Dai tratti di un modello agrario: produzione, risparmio, commercio, sistema dei tributi e delle infrastrutture, sistema economico.

Davanti al sistema attuale di produzione anarchica e poco umana, che contrappone la "società dello sperpero" all'insoluto dramma "Nord-Sud", Müller dichiara fallito ogni modello: quello dello "Stato del benessere» [o "capitalismo socialemente moderato"], quello dell'ordo-liberalismo e quello di "pianificazione di congiuntura". 
a lungo un sistema politico con le proprie istituzioni costituzionali e strutture legali può resistere dinnanzi a fattori sfavorevoli, specie la crisi economica non di breve periodo? In termini positivi: un sistema democratico può consolidarsi definitivamente quando il Paese trova un modello di riproduzione socio-economico stabile.

Diamond riconosce come il consolidamento democratico sia intimamente legato a riforme economiche strutturali. ${ }^{260}$ Drastici cambiamenti tra politiche populiste redistributive e politiche di neoliberal austerity sollecitano miserie e crisi economiche del tipo di quelle che minacciano il futuro della democrazia in Argentina, Brasile e Perù.

Due assunti: (a) le democrazie non producono performances economiche peggiori rispetto a quelle delle dittature; neppure in sè garantiscono performances economiche migliori. Le politiche scelte e le capacità con cui sono implementate sono molto più importanti; (b) essendo la coerenza, la prudenza e il pragmatismo in politica così importanti per lo sviluppo economico, le nuove democrazie debbono curare come formare e mantenere un largo consenso sulla politica economica, il che richiede creatività nell'ideare istituzioni, educazione pubblica, accomodamento delle élite e soprattutto una leadership politica dotata di coraggio, intuito e determinazione.

\subsection{La concezione sociologica}

Manuel Antonio Garretón afferma la necessità per il consolidamento di fattori della "democratización social o global", quali il superamento della marginalizzazione, la ridistribuzione, l'eguale accesso alle opportunità, l'eliminazione delle diseguaglianze. ${ }^{261}$ Fondamentale è, anzitutto, la creazione di canali di integrazione per la disintegrazione sociale.

Il consolidamento è legato, quanto al punto di partenza, alla "democratización política completa». ${ }^{262}$ In America Latina l'idea di democrazia è

260 Diamond, L., "Three paradoxes of Democracy», in: Diamond L., Plattner M. F. (a cura di), The Global Resurgence of Democracy, op. cit., pp. 95-107, già in: JoD, vol. 1.3, 1990.

261 Garretón Merino, M. A., La posibilidad democrática en Chile. Dilemas de Transición y Consolidación, Flacso, Documento de Trabajo N 394, Santiago de Chile, 1988.

262 Valenzuela critica l'ambizione di una ricostruzione a livello di ogni genere di relazione e istituzione politica e sociale. 
sempre stata legata ad un principio etico di integrazione o democratizzazione sociale, vale a dire di eliminazione delle diseguaglianze e di participazione alle decisioni che interessano «la gente». ${ }^{263}$

Per Garretón non è sufficiente che sia scomparso il rischio di un rovesciamento del regime democratico, ma è necessario individuare le condizioni -diverse da Paese a Paese: non c'è determinismo- ${ }^{264}$ di stabilità della democrazia. Non si possono fissare condizioni per il consolidamento (siano esse la crescita economica, l'esistenza di determinate istituzioni o valori, una data composizone della struttura delle classi sociali, una specifica configurazione di fattori internazionali): sono i regimi democratici fattori favorevoli allo sviluppo di tali fenomeni e non viceversa (Schmitter e Karl). ${ }^{265}$

Consolidamento e stabilità sono correlati a tre fattori, non necessariamente concomitanti:

a. la «ricostruzione nazionale», vale a dire la ridefinizione del modello di sviluppo —non solo economico-, capace, essendo includente, di garantire la rilevanza della prospettiva di democratizzazione per i diversi attori socio-politici ["democratización global"];

b. la forma di organizzazione sociale, la matrice d'articolazione tra Stato (capace di gestire, attore nazionale di fronte ai poteri esterni e agente principale di ricostruzione della società, anche mediante un ampliamento e una estensione delle istituzioni statali), sistema politico (capace di

263 Carretón, M. A., "La democracia entre dos épocas", in: Hacia una nueva era política. Estudio sobre las democratizaciones, Santiago, 1995, p. 46.

Tre sono le questioni : l'esclusione/ integrazione sociale e la frammentazione/coesione sociale; l'espansione della cittadinanza [c.d. "democratización fundamental»]; la partecipazione. Cfr. Garretón M. A., "Democratización, desarrollo, modernidad: ¿Una nueva problemática para América Latina?", in: Alcántara M. e Crespo I., (a cura di), Los limites de la consolidación democrática en América Latina, op. cit., pp. 75-82.

264 Auspica pertanto l'analisi per un numero ristretto di casi. Vedi Garretón M. A., "Democracia, transición y consolidación, un esquema general", in: Reconstruir la politica. Transición y consolidación democrática en Chile, Santiago, 1987, p. 52 y ss.

$265 \mathrm{Karl}$, T. L. e Schmitter P. C., "Modes of Transition in Latin America, Southern and Eastern Europen, in: International Social Science Journal, 1991, vol. 128, p. 270, già Schmitter, P. C. e Karl, T. L., What kind of Democracies are Emerging in South America, Central America, Southern Europe and Eastern Europe?, Stanford, 1991.

266 Carretón, M. A., "La democracia en Chile, desarrollo, crisis y perspectivas", in: Reconstruir la política. Transición y consolidación democrática en Chile, Santiago, 1987, p. 89 y ss. 
rappresentare, anche a mezzo di attori e movimenti sociali non tradizionali, di spazi sociali e culturali di espressione non partitici) e società civile (garantendo crescente autonomia agli attori sociali rispetto al sistema partitico di modo che il frazionamento e la polarizzazione politica non si riproducano automaticamente nel contesto sociale);

c. la strutturazione del sistema partitico, tale da essere non escludente, da raccogliere l'adesione democratica in tutti i punti dello spettro politico, capace di creare coalizioni e una maggioranza socio-politica che combini la adesione democratica con una promessa di riforme sociali per un lungo periodo, e dotato di legittimità interna svincolata dal conseguimento di particolari risultati. La maggioranza [«bloque democrático transformadon»] deve avere un orizzonte culturale di larghe vedute, che non elimini le diversità culturali, nè la eterogenirà sociale e che, pertanto, presuppone interessi parzialmente contradditori che devono esser risolti nella competizione democratica. ${ }^{266}$

Il consolidamento, se legato ai detti tre fattori $[a, b, c]$, richiede un cambio nello stile e nella cultura politica.

Per completare la transizione e iniziare il consolidamento, compiti simultanei e non in sequenza cronologica, secondo Garretón, è necessario un governo maggioritario non solo politicamente ed elettoralmente, ma anche socialmente e istituzionalmente [1]. Il consolidamento è associato ad un fattore storico-politico qual è la desiderabilità democratica da parte degli attori sociali significativi [2]. Tale desiderabilità —almeno per l'America Latina- è alimentata dal principio fondamentale dell'integrazione e democratizzazione sociale [3]. ${ }^{267}$

\subsection{La concezione semantica [Il pentaedro di Schedler]}

Andreas Schedler ${ }^{268}$ denuncia la incomunicabilità nascosta dietro al paravento di una medesima etichetta: "consolidamento democratico». ${ }^{269}$ Piuttosto che al "consolidamento", meglio sarebbe riferirsi a "tipi di consolidamento».

267 Carretón, M. A., "La democracia entre dos épocas", in: Hacia una nueva era política. Estudio sobre las democratizaciones, Santiago, 1995, p. 49 e 105.

268 Schedler, A., Concepts of Democratic Consolidation, Latin American Studies Association (LASA), Wien-Guadalajara, aprile, 1997, in seguito largamente rielaborato come "What is Democratic Consolidation?", in: JoD, vol. 9.2, 1998, pp. 91-107.

269 "The aspiring subdiscipline of "consolidology" is anchored in an unclear, inconsistent, 
A complicare l'uso disinvolto che ne fanno gli autori, l'ambiguità semantico-etimologica del termine "consolidamento" si riferisce sia al processo [consolidating democracy] che al conseguimento dell'agognato risultato [consolidated democracy]. Fino a far collassare i due campi e a definire il risultato mediante il processo, ossia a definire il consolidamento mediante "what it takes to achieve it".

All'interno di un approccio teleologico, ${ }^{270}$ Schedler si ripropone di far chiarezza nella commistione di direzioni che al consolidamento vengono impresse. Per argomentare si avvale di un artificio non del tutto cristallino (per tacer dell'utilizzo discutibile di quattro idealtipi di regime): la creazione di cinque sotto-concetti di consolidamento democratico.

L'analisi dell'Autore austriaco si snoda lungo due coordinate:

- sulle "ascisser(", il contesto [where we stand: our empirical viewpoint]: il tipo di regime politico che stiamo considerando. Si tratta della democrazia elettorale (o semidemocrazia) e della democrazia liberale, giacchè solo la parte centrale dello schema è suscettibile di movimento in termini di "consolidamento";

- sulle "ordinate», il fine [where we aim to reach: the normative horizons]: il tipo di regime politico che vorremmo raggiungere o evitare. Si tratta di forme diminuite o incrementate di democrazia, che vedono agli estremi l'autocrazia e la democrazia avanzata.

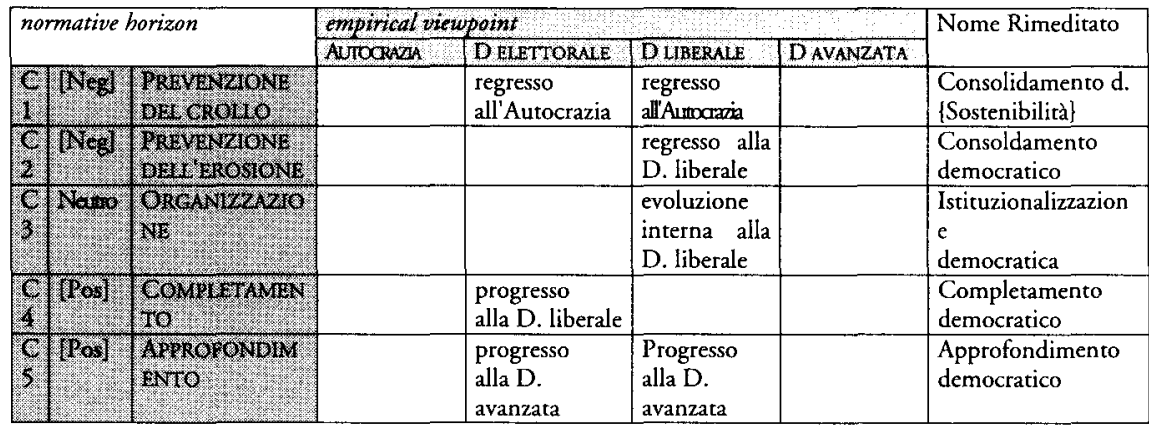

and unbounded concept, and thus is not anchored at all [but drifting in murky waters]". Il linguaggio comune [un caso di homonymity] sarebbe solo simulazione, laddove regna il disordine concettuale. "As matter now stand, the concept's classificatory utility is close to zero", conclude sotto la rubrica Post-Transitional Blues.

270 «Consolidamento democratico» è intrinsecamente un concetto teleologico. Il che non va confuso con la necessarietà, con il percorso inevitabile, la naturalità o automaticità. 
Il consolidamento ha una definizione dicotomica:

negativa se concerne la stabilità democratica e la prevenzione del regresso;

positiva se concerne l'avanzamento democratico e il perseguimento del progresso.

In altri termini: positiva se attiene al raggiungimento della continuità democratica, negativa se descrive il superamento della fragilità democratica.

É dato tuttavia un uso neutro del concetto: si ottiene all'interno della democrazia liberale, considerando che per consolidare non basta istituzionalizzare le procedure e organizzazioni minime, ma occorre stabilire specifiche regole e istituti ${ }^{271}$ (il che potrebbe, volendo, accedere all'identificazione di diversi tipi di democrazia). Occorre la istituzionalizzazione sociologica (laddove per consolidamento s'intende il divenire degli attori sempre meno coscienti della contingenza delle istituzioni democratiche) ${ }^{272}$ e l'istituzionalizzazione sub-sistemica (laddove il consolidamento si concreta nella creazione di specifiche istituzioni di meso o micro livello).

La nozione di "consolidamento democratico" contempla, dunque, molti telo $i$ caratteristici: tale pluralità di telo $i$ conduce a una pluralità di concetti di consolidamento democratico.

Tuttavia Schedler rinviene una radice semantica: il termine "consolidamento democratico" dovrebbe riferirsi alle aspettative di continuità per il regime e a nient'altro. In altri termini, il consolidamento è quello, per così dire, «classico» ${ }^{273}$ (sub 1-), attinente al raggiungimento di un livello di governo tale da tutelare da regressi autocratici; è, in definitiva, estendibile al massimo sino a quello indicato dalle due prospettive «negative».

271 Tra quelli che Schmitter chiama "partial regimes", Schedler indica: i partiti e il sistema partitico, le assemblee legislative, la burocrazia statale, il sistema giudiziario, i sistemi d'intermediazione degli interessi.

272 In complesso, tre dimensioni concorrenti: acquiescenza strategica [compatibilità stimolante, negli scritti di Di Palma], consenso normativo [legittimazione, negli scritti di Linz, Stepan e Munck] e consapevolezza irriflessiva [Schedler].

273 Quanto a thin conceptualization, un regime è consolidato quando ha probabilità di perdurare (O'Donnell, G., "Illusions about Consolidation», in: JoD, vol. 7.2, 1996) ovvero quando si spera prosperi nel futuro (Valenzuela J. S., Democratic Consolidation in Post-Transitional Settings: Notion, Process, and Facilitating Conditions, Kellogg Working Paper No 150, dicembre, 1990). 
Può sostenersi un aggiramento del problema, consultando l'opera di Schedler che in chiusura si arrende e utilizza etichette diverse per i cinque consolidamenti. Ma la vera resa è riscontrabile allorchè l'Autore si limita in altre opere alla trattazione del consolidamento in senso "classico", abbandonando la difficile (e intagliata con difficoltà) concettualizzazione pentapartita. ${ }^{274}$

\subsection{Le concezioni negatrici}

In limine, va ricordato che non implica negazione del concetto il fatto che alcuni Autori ricorrano ad etichette diverse e/o evitino di ricorrere esplicitamente al termine "consolidamento", oppure, come talora si verifica, utilizzino implicitamente il termine senza darne una definizione. ${ }^{275}$

Vi sono, invece, talune prese di posizione radicali.

\subsubsection{Gli autori possibilisti}

a) La negazione ontologica: la continua capacità di mutare.

Il binomio "consolidamento democratico" pare una contraddizione in termini: si ritiene che le democrazie mai siano completamente consolidate: dovrebbero contenere il germe, la potenzialità per [e la necessità di] un continuo cambiamento. Ma verità vuole che de facto modelli e norme vengano strutturati in modi altamente previsibili e persistenti, tanto che de jure diviene difficile cambiarli. ${ }^{276}$

b) La negazione empirica. Non regge alla prova dei fatti.

Dharragh Hunt riconosce che "consolidamento" è concetto problematico. Anzitutto non è così scontato che i Paesi stabiliscano meccanismi formalmente democratici su sistemi non-democratici.

274 Schedler, A., "Cómo observar la consolidación democrática?», in: Metapolitica, vol. 15, 2000 ; Idem, "Measuring Democratic Consolidation", in: Studies in Comparative International Development, vol. 36.1, 2001, pp. 66-92.

275 Per esempio, in senso istituzionalista, Agh A., Democratic institution-building in East Central Europe and the Balkans, op. cit., pp. 1-33.

276 Schmitter, "The Consolidation of Political Democracies: Pracesses, Rhythms, Sequences and Types, in: Pridham G. (a cura di), Transitions to Democracy. Comparative Perspectives from Southern Europe, Latin America and Eastern Europe, op. cit., pp 535-569. 
Termini quali consolidato/non consolidato o democrazia liberale/ democrazia elettorale probabilmente non sono sufficienti a spiegare la estensione della varietà dei casi concreti che «alla fine della terza ondata di democratizzazione" possono esser considerati. ${ }^{277}$

c) Durata connessa al regime economico in via quasi assoluta.

Per Adam Przeworski, ${ }^{278}$ fautore del ruolo della economic performance, il concetto di consolidamento è privo di significato se guarda al rafforzamento di norme e istituzioni in collegamento al trascorrere del tempo. ${ }^{279}$

d) La difficoltà per oscurità: la resa.

Pòstane in dubbio la reale utilità, alcuni Autori propendono esplicitamente per la sostanziale superfluità del concetto, tacciato di eccessiva vaghezza.

Schneider dichiara necessario disaggregare il concetto composito di consolidamento [e di regime democratico], unica alternativa allo scartarlo. Sarebbe bene, allora, focalizzare l'attenzione su come le parti componenti operino, piuttosto che cercare di affermare se un certo sistema politico nel complesso sia consolidato. Di fronte alle difficoltà definitorie, meglio pensare il consolidamento come un tema secondario di ricerca. ${ }^{280}$

Schedler ${ }^{281}$ chiarisce la tentazione di abbandonare il concetto di consolidamento. Si tratta di una seconda scelta: meglio sarebbe per gli Autori contenersi e utilizzare «nomi differenti per cose diverse», vale a

277 Hunt, D., Transitology and Political Change in Russia. Russian and East European Politics, Helsinki, 2001.

278 Przeworski, A., Alvarez, M., Cheibub, J. A., Limongi, E., «What makes Democracies Endure?", in: JoD, vol. 7.1, 1996, pp. 39-55. "Consolidation is an empty term», non avendo riscontrato casi in cui il mero trascorrere del tempo abbia reso il rovesciamento della democrazia meno probabile, 51.

279 Prima facie, questo parrebbe l'orientamento di Huntington, quando tratta del consolidamento sotto l'infelice rubrica di "La durata [della democrazia]" : Huntington, S. P., The Third Wave. Democratization in the Late Twentieth Century, op. cit., cap. V. Sicuramente confondono consolidamento e durata Power T. J. e Gasiorowski M. J., "The Structural Determinants of Democratic Consolidation. Evidence From the Third Worlds, in: Comparative Political Studies, vol. 31.6, 1998, pp. 740-771.

280 Schneider, B. R., "Democratic Consolidations: Some Broad Comparisons and Sweeping Arguments", in: $L A R R$, vol. 30.2, 1995, pp. 215-234. 
dire smettere di usare il termine "consolidamento" per qualsiasi cosa si vorrebbe accadesse nelle nuove democrazie (le condizioni del consolidamento) o per qualsiasi cosa si ritenga problematica per tali regimi (i problemi del consolidamento).

O'Donnell prende posizione, nell'alternativa: le ricerche dovrebbero esser tese a "gettare a mare il concetto", piuttosto che a tentare di chiarirlo. ${ }^{282}$

Munck sostiene l'opportunità di abbandonare «l'ingombrante riferimento a differenti versioni del concetto di consolidamento democratico" e dirottare l'attenzione su due concetti: quello di stabilità democratica e quello di qualità democratica. ${ }^{283}$

\subsubsection{L'«abiura» di O’Donnell}

L'Autore argentino spiega a chiare lettere nel $1999^{284}$ : i suoi "primi" lavori sulle nuove democrazie ${ }^{285}$ davano per fondata l'esistenza di un corpus di teoria democratica sufficientemente chiaro e consistente. A distanza di pochi anni (1988-1996) fa pubblica abiura delle allora condivise vedute sul consolidamento democratico.

Non giova dal punto di vista analitico, in contrasto sul punto con Linz, aggiungere l'attributo di «consolidata» a qualcosa che probabilmente resisterà, ma non è sicuro lo faccia. "Democrazia e consolidamento sono

281 Schedler, A., "What is Democratic Consolidation?", in: JoD, vol. 9.2, 1998, pp. 91-107.

282 Forse i problemi correlati al concetto di consolidamento sono tanto profondi da aver resa esausta la sua utilità : O’Donnell, G., “Illusions about Consolidation», in: $J o D$, vol. 7.2, 1996, pp. 34-51. Per un dibattito conseguente vedi Gunther R., Diamandouros P. N. e Puhle H.-J., "O'Donnell's "Illusions»: a Rejoinden, in: JoD, vol. 7.4, 1996, pp. 151 159 ; O'Donnell, G., "Illusions and Conceptual Flaws, in: JoD, vol. 7.4, 1996, pp. 160 168.

283 Munck, G. L., Democracy Studies: Where to From Here?, Urbana-Champaign, 2001 .

284 O'Donnell, G., Democratic Theory and Comparative Politics, Wissenschaftszentrum Berlin für Sozialforschung [WZB] Paper 99/004, Berlin, ottobre, 1999.

285 Il mea culpa s'appunta in particolare su «Transitions, Continuities, and Paradoxes", in: Mainwaring, S., O'Donnell, G. e Valenzuela, S., (a cura di) Issues in Democratic Consolidations: The New South American Democracies in Comparative Perspective, op. cit., pp. 17-56, in realtà già edito in Wanderley Reis, F. e O'Donnell, G., (a cura di), A democracia no Brasil: dilemas e perspectivas, São Paulo, 1988. 
termini troppo polisemantici per formare una buona coppia». ${ }^{286} \mathrm{~A}$ poco giova la "the only game in town" definition, se è vero, come è vero, che Linz e Przeworski fanno riferimento esclusivo alle regole formali della democrazia, se fondamento del consolidamento è l'equilibrio tra regole formali e condotta reale.

e) La negazione interpretativa teleologica

O'Donnell rintraccia nella letteratura sul consolidamento democratico la recondita congettura dell'esistenza di un cammino naturale e uno stato finale propri dello sviluppo democratico.

Chiarisce Diamond: il concetto di consolidamento democratico è significativo ed utile solo al denotare una soglia di legittimità e stabilità politica che non è irreversibile. Anche consolidata, la democrazia può rafforzarsi quanto indebolirsi sempre, in ogni momento farsi più o meno piena. ${ }^{287}$

\section{f) La concezione individualizzante}

O'Donnell ${ }^{288}$ critica il tentativo di misurare le democrazie realmente esistenti sulla base di modelli, anche empirici, cui implicitamente si ritiene che esse debbano prima o poi corrispondere. Vanno esaltati invece i tratti originali di ogni ordinamento, la specificità delle regole e delle istituzioni che presiedono al funzionalmento delle nuove democrazie: avremmo i tratti inediti dei nuovi regimi. Con una venatura polemica, nel 1996 sostiene che le principali definizioni esistenti di consolidamento implicitamente descrivono le democrazie nord-atlantiche e sono pertanto vulnerabili a teleologiche asserzioni, per le quali le democrazie dei Paesi emergenti arriveranno a configurarsi come le nord-atlantiche e a mezzo di percorsi già da queste sperimentate. ${ }^{289}$

286 O’Donnell, G., «Illusions about Consolidation», in: JoD, vol. 7.2, 1996, pp. 34-51. 287 Quando la democrazia ristagna e i suoi cittadini si fanno politicamente apatici e indifferenti, è suscettibile di deteriorarsi. «Il prezzo della libertà è in effetti una eterna vigilanza»: Diamond L., "El final de la tercera ola y el futuro global de la Democracia», in: López E., Mainwaring S. (a cura di), Democracia: discusiones y nuevas aproximaciones, Buenos Aires, 2000, nota 48.

288 O’Donnell, G., Delegative Democracy?, Kellogg Institute, Working Paper No 172, New Haven, marzo, 1992, anche in: JoD, vol. 5.1, 1994, pp. 55-69.

289 O'Donnell charisce che le nuove democrazie dell'Est e del Sud potrebbero avere un volto assai diverso da quelle di Europa e Nordamerica : O'Donnell, G., Illusions about Consolidation, op. cit., p. 43. 
Va ricordato che in quest'ottica, Diamond, Linz e Lipset rifiutano di fornire un "nuovo, elegante, parsimonioso modello" esplicativo e optano per l'illustrazione dei fattori storici, culturali, sociali, economici, politici ed internazionali che possono determinare la stabilità delle democrazie. ${ }^{290}$

\section{I soggetti: gli attori del consolidamento}

Essendo il consolidamento un processo, è «obbligatorio concentrare l'analisi sugli attori in quanto potenziali agenti di consolidamentom. ${ }^{291} \mathrm{I}$ soggetti principali sono:

1. i partiti, essendo quasi insostituibile la loro funzione di intermediazione;

2. i gruppi d'interesse, per la rappresentazione funzionale;

3. la società civile. Rilevano qui spazi pubblici di carattere autonomo $\mathrm{e}$ interrelazioni. La società è elemento articolatore degli interessi cittadini e si rivela indispensabile per raggiungere la complementarietà ${ }^{292}$ necessaria tra le istituzioni e gli attori sociali e politici. Il consolidamento è caratterizzato da un rinnovato protagonismo della società civile. Non è sostenibile a lungo termine una «democracia sin demócratas". Ma Rainer Eisfeld indica "il paradosso del consolidamento" nel fatto che la rivitalizzazione della società civile non ha automaticamente e comunque effetti positivi se non in una fase avanzata del consolidamento, «nonostante sia proprio la società civile ad agevolare lo stabilizzare e il democratizzare la democrazia nel lungo terminem. ${ }^{293}$

La prima frattura interpretativa si ha tra gli scritti che prediligono una trattazione incentrata sulle élite e opere che contemperano le stesse con l'agire delle masse. ${ }^{294}$

290 Diamond, L., Linz J. J. e Lipset S. M., "Prefaces", in: Democracy in Developing Countries, Tomo I, Politics in Developing Countries: Comparing Experiences with Democracy, Boulder, 1988, p. xiii.

291 Morlino, L., Consolidamento democratico: definizione e modelli, op. cit., p. 223.

292 Misurata attraverso il consenso ai meccanismi democratici.

293 Eisfeld R., The Role of Elites and Masses in the Transition from Autocratic to Democratic Regimes, International Political Science Association [IPSA] \& Queen's University, Kingston [Ontario], 1998.

294 Plasser, F., Ulram P. A. e Waldrauch H., "The Concept of Consolidation in Regime Change Research", in: Democratic Consolidation in East-Central Europe, HoundmillsLondon, 1998, p. 45. 
Non solo le élite, come troppo spesso ormai la scienza inclina a fare, ${ }^{295}$ vanno considerate, ma anche il comportamento della "gente comune». ${ }^{296}$ Il consolidamento richiede che «i cittadini sviluppino un apprezzamento per le istituzioni fondamentali di una società politica democratica: partiti, parlamento, elezioni, leggi elettorali, leadership politica e coalizioni" ${ }^{297}$

Venendo ai meccanismi di rappresentazione, secondo nodo interpretativo, taluno definisce il consolidamento un "party-dominated processm. ${ }^{298}$ Se questo è l'essere, quanto al dover essere, Ricardo Córdova e Günther Maihold ${ }^{299}$ dichiarano invece necessario che i partiti condividano gli spazi di intermediazione sociale con attori della società civile e i gruppi d'interesse: predicano la complementarietà tra rappresentazione territoriale (partitica) e rapprensentazione funzionale (gruppi d'interesse) come una delle mete centrali del processo di consolidamento. ${ }^{300}$ Mentre può esser calzante per il periodo di transizione, ${ }^{301}$ per il consolidamento non ci si

295 Per tutti, Higley J. e Gunther R. (a cura di), Elites and Democratic Consolidation in Latin America and Southern Europe, Cambridge, 1992.

296 Ardouin, S., Parties and the improvement of democracy: A framework for analysis, Newcastle-upon-Tyne, 1998. Boussard avverte come una spiegazione élite-centred sia insufficiente almeno per comprendere il consolidamento "positivo in prospettiva evolutiva" : Boussard C., Civil Society in the Consolidation Process. Illustrations from Central America, ECPR XXVIII Joint Sessions of Workshops, Workshop 4, Copenhagen, 2000.

297 Linz, J. J. e Stepan, A., "Towards Consolidated Democracies, in: JoD, vol. 7.2, 1996, p. 17, successivamente in Inoguchi T., Newman E. e Keane J., (a cura di), The changing nature of democracy, op. cit., pp. 48-68.

298 Pasquino, G., "Party Elites and Democratic Consolidation: Cross-national Comparison of Southern European Experiencen, in: Pridham G. (A cura di), Securing Democracy: Political Parties and Democratic Consolidation in Southern Europe, London, 1990, p. 52. Così Ardouin S., Parties and the improvement of democracy: A framework for analysis, op. cit.. Contra Morlino, L., per il quale il modello incentrato su partiti non è che un tipo di spiegazione del consolidamento. Contra Schmitter P. C., che distingue gli attori nelle diverse fasi del processo temporale di democratizzazione.

299 Córdova, R. \& Maihold, G., Democracia y ciudadanía en Centroamérica. Perspectivas hacia el 2020, CA2020: Documento de trabajo No 9, Institut für Iberoamerika-Kunde, Hamburg, 2000.

300 Spesso forti movimenti sociali e gruppi d'interesse tendono a bloccare le riforme economiche o a schierarsi lungo fratture sociali.

301 Ancora Schmitter ritaglia ulteriormente quanto in precedenza affermato assieme a O’Donnell. Vedi O'Donnell, G. e Schmitter, P. C., "Convocatoria a elecciones (e incitación a los partidos políticos)", in: O'Donnel,1 G., Schmitter, P. C., Whitehead, L., Transiciones desde un gobierno autoritario, versione in castigliano coordinata da Oszlak O., Tomo IV, 
può limitare alla considerazione del terreno elettorale e partitico. Significherebbe sottostimare il ruolo dei gruppi d'interesse ${ }^{302}$ nel condurre legittimazione. ${ }^{303}$

Gli attuali consolidamenti democratici avvengono in un ambiente saturo di organizzazioni [a], denuncia Schmitter. Esse richiedono uno status legale [b], dovendo controllare le risorse, abbisognano di autonomie e accesso per funzionare effettivamente nella competizione per gli uffici o l'influenza. ${ }^{304}$

Chiaramente $\mathrm{i}$ "modelli convertiti in strutture» dal consolidamento non possono limitarsi ad esistere in funzione di una legge: devono esser valutati sia da chi agisce al loro interno, sia da chi è affetto da loro. È la legittimazione delle strutture delle nuove democrazie. Pur tra notevoli difficoltà innate:

- le democrazie esaltano le preferenze individuali e le scelte volontaristiche;

- le democrazie sarebbero basate sul principio di eguaglianza, ma i gruppi assegnano ruoli e benefici in modo differenziato;

- le democrazie dipendono da competizione, sfida e libera informazione, elementi che minano l'apparente naturalità e desiderabilità delle istitutizoni esistenti (poichè consentono di svelarne i difetti e di proporre arrangiamenti alternativi);

- le democrazie generano stretti legami tra i gruppi nella società civile e le agencies dello Stato. Ma ciò mina l'autonomia operativa degli enti e sovverte la loro dichiarata difesa dell'interesse generale;

Conclusiones tentativas sobre las democracias inciertas, Barcelona-Buenos Aires, 1988 (1994), cap. 6, già Transitions from Authoritarian Rule, Tomo IV, Tentative Conclusions about Uncertain Democracies, Baltimore-London, 1986.

302 I movimenti sociali di base, così determinanti al tempo della transizione, non scompaiono, nonostante le loro energie e tematiche siano assorbite dai partiti, gruppi e agencies pubbliche specializzate : Schmitter P. C., "The Consolidation of Democracy and Representation of Social Groups", in: American Behavioral Scientist, No 35, 1992, pp. 422-449.

303 Schmitter, P. C., "Organized Interests and Democratic Consolidation in Southern Europen, in: Gunther R., Diamandouros N. e Puhle H.-J. (a cura di), The Politics of Democratic Consolidation. Southern Europe in Compatative Perspective, op. cit., pp. 284-314.

304 Schmitter, P. C., The Consolidation of Democracy and Representation of Social Groups, op. cit., pp. 422-449. 
- le democrazie incoraggiano l'aperta espressione di conflitti di interessi entro i gruppi governanti, il che distrugge la capacità dei membri di un'azione unificata;

- la democrazia distribuisce, assegna i diritti egualmente, mentre il capitalismo concentra il diritto di proprietà in modo non equo.

Nelle democrazie, il luogo in cui il consenso è estratto e si riproduce è il sistema dei partiti.

Date queste due premesse [a,b], si rivelano centrali per il consolidamento il Parlamento e i gruppi d'interesse. ${ }^{305}$ Il Parlamento, infatti, non solo è chiamato a strutturare sè stesso, ma ratifica molte delle norme che strutturano altre istituzioni democratiche. ${ }^{306}$

Ulrike Liebert propende per la centralità del Parlamento in almeno un tipo di consolidamento. ${ }^{307}$ Di Palma qualifica il Parlamento sia insufficiente [«institutionally insufficient»] che sovrastimato ["an unnecessary surplus"], rispetto alla soluzione dei problemi connessi al consolidamento. ${ }^{308}$

\section{I fattori del consolidamento}

Vi sono trattazioni del consolidamento che, sottraendosi ad ogni sforzo teoretico, si limitano a descrivere un certo numero di fattori, quasi il consolidamento fosse un'espressione algebrica, ${ }^{309}$ la risultante di una serie di addizioni e sottrazioni di elementi.

305 Come nella deposizione dell'autoritarismo centrali erano i movimenti sociali e, invece, nella transizione i partiti.

306 Schmitter stima che i Parlamenti richiedano tre legislature complete (esclusi quindi gli scioglimenti anticipati) per completare la propria strutturazione e approvare quella di altre istituzioni.

307 Bene indicava Morlino come il consolidamento ventrato sul Parlamento è solo uno e relativamente raro [Italia 1946 e Spagna 1977] dei molti tipi di consolidamento. Basti pensare a come sia rimasto in sordina in Argentina. Cfr. Liebert U., The Centrality of Parliament in the Consolidation of Democracy: $A$ Theoretical Exploration, ICPS, Working Paper No 7, Barcelona, 1989. Già Schmitter (1988) aveva ipotizzato che il Parlamento è il luogo in cui è raggiunta la legittimazione per il nuovo regime.

308 Vedi Liebert U. e Cotta M., (a cura di), Parliament and Democratic Consolidation in Southern Europe, London, 1990.

309 Eloquente Grassi che applica un'equazione tra il consolidamento e una formula booleana. Cfr. Grassi D., La Democrazia in America Latina. Problemi e prospettive del consolidamento democratico, Milano, 1999. 
Ed esiste una certa facilità in dottrina ad etichettare come "fattore" ogni tipo di variabile del consolidamento, trascurando la natura di «elemento fautoren che invece dovrebbe distinguere i fattori. Meglio sarebbe, a questo punto, scriminare tra fattori che determinano il consolidamento ed elementi che influenzano il consolidamento. La rilevazione empirica rischierebbe di rivelarsi soggetta a particolarismo, di variare da ordinamento a ordinamento, ma sarebbe tale da apportare un innegabile progresso teorico.

Non va da sé che i fattori siano anche le determinanti del consolidamento.

$\mathrm{Va}$ infine detto che si deve operare ben lungi da ogni parvenza di determinismo. Nelle parole di Philippe Schmitter, né il livello di sviluppo economico, lo stato di accumulazione capitalista, nè l'egemonia della borghesia possono automaticamente garantire l'avvento e meno ancora la persistenza della democrazia. Nè tale regime è il prodotto inevitabile di alcuni livelli di "civilizzazione», alfabetizzazione, livello educativo o cultura politica. Ciò che su tutto quanto sopra rileva è che la democrazia deve essere scelta, implementata e perpetuata da agenti politici con propri interessi, passioni, memorie, «fortuna» e «virtù»: ad essi (a prescindere da fattori che inevitabilmente li condizionano) spetta compiere scelte corrette o sbagliate.

Sarebbe tuttavia fuorviante incentrare il tutto sulla capacità di scelta $e$ l'azione volontaristica.

Nel breve, il consolidamento della democrazia dipende dall' abilità di attori e cittadini di individuare una soluzione per $\mathrm{i}$ loro intrinseci conflitti sulle regole [«interesse dei politici»].

Nel lungo periodo, dipenderà dall'estrinseco impatto che politiche elaborate sotto l'egida di quelle regole avranno sui gruppi sociali [«aspettative dei cittadini»]. $\mathrm{E}$ ' in tale sede che intervengono le "realtà oggettive" (livelli di sviluppo, posizioni nell'economia mondiale, conflitti su prodotti settoriali e distribuzione di benessere) e le "preferenze soggettive» (di classi, generazioni, generi, tipi etnici, gruppi di status).

Esistono, dunque, fattori che rendono più agevoli i processi di consolidamento.

In limine, una osservazione, che va letta in negativo, di Gurutz Jáuregui: oggigiorno le sfide principali ai sistemi democratici [già] consolidati non provengono dall'esterno, quanto dalla loro stessa struttura e 
funzionamento. ${ }^{310}$ "Le sorti delle democrazie [sono] legate in primo luogo alla volontà dei leader politici di proseguire» la strada del rafforzamento, «di pagarne $\mathrm{i}$ costi e di non dare la priorità ad altri obiettivi». ${ }^{311}$

A mero titolo d'esempio, seguono le elencazioni fornite da alcuni politologi.

Morlino prospetta alcuni fattori che, secondo la sua ricostruzione, facilitano il consolidamento:

1. un rapido, ampio e completo spettro di partiti che sorga e si organizzi durante la transizione;

2. il protagonismo durante la istaurazione delle forze democratiche di destra o di centro-sinistra;

3. una cultura politica poco radicalizzata, nonostante la memoria storica per i costi sofferti durante il periodo autoritario o le profonde trasformazioni socio-economiche occorse durante lo stesso;

4. la strutturazione delle organizzazioni di interesse della società civile, in particolare, il fatto che le associazioni imprenditoriali e sindacali accettino una soluzione democratica;

5. l'assenza di problemi di costruzione dello Stato;

6. l'esistenza di una struttura amministrativa e giudiziaria;

7. l'omologazione internazionale a seguito dell'adesione a organizzazioni sovranazionali.

Michael Burton, Richard Gunther e John Higley $(1992)^{312}$ vorrebbero sottrarsi ad un modello esplicativo monocausale, un "élite-determinism».

Fattori determinanti il consolidamento sono, accanto ai fondamentali élite settlement e/o élite convergence, l'esistenza di una cultura e di una tradizione politica democratica (e in essa la pressione delle masse per la democrazia) e il livello di sviluppo socio-economico. Tali fattori, una volta elencati, sono tuttavia ancora una volta piegati alla necessità di unificare le élite o ingenerarne il consenso democratico.

310 Jáuregui, G., Problemas actuales de la democracia, Universitat Autónoma de Barcelona, Institut de Ciències Polítiques i Socials [ICPS], Working Paper No 119, 1996.

311 Huntington, S. P., La terza ondata. I processi di democratizzazione alla fine del XX secolo, op. cit., p. 294.

312 Burton, M., Gunther, R. e Higley, J., "Elites and democratic consolidation in Latin America and Southern Europe: an overviewn, in: Higley J. e Gunther R. (a cura di), Elites and Democratic Consolidation in Latin America and Southern Europe, op. cit., pp. 323- 348. 
Linz e Stepan, insieme con Gunther, ${ }^{313}$ indicano tra le variabili che interessano transizione e consolidamento: la natura del precedente regime non-democratico, la complessità derivante dall'avere intrapreso simultaneamente radicali riforme economiche, sociali, legali e burocratiche in tempo di transizione, ${ }^{314}$ la questione della statualità, la forza e l'interna composizione dei governatnti del regime uscente, il problema di chi inizia e chi controlla la transizione, le influenze internazionali e lo stile del processo costituente. Diamandouros, Puhle e Gunther indicano successivamente: il legame tra fattori socio-economici e democrazia, l'eredità storica ed in specie la natura del regime che precedette quello autocratico, i caratteri peculiari della transizione, i partial regimes. ${ }^{315}$

\section{Tabella: I fattori più ricorrenti nella letteratura}

\begin{tabular}{|c|c|c|}
\hline & FATTOR DICONSOLIDAMENTO & AUTORE DI RIFERIMENTO \\
\hline istituzionali & $\begin{array}{l}\text { permanenza di tradizioni e istituzioni pre- } \\
\text { autocratiche } \\
\text { tipo e durata del regime autocratico } 1 \text { - } \\
\text { modalità di Transizione } \\
\text { collocazione temporale } \\
\text { forma di stato } \\
\text { forma di governo } \\
\text { opzione federale } \\
\text { Sistema elettorale } \\
\text { persistenza di alegati autoritarim } \\
\text { subordinazione dei militari ai civili } \\
\text { Sistema di intermediazione } \\
\text { Sistema partitico 2- } \\
\text { decision-making system } \\
\text { la moderazione del conflitto politico } \\
\text { gestione del conflitto sociale } \\
\text { efficacia governativa 3- }\end{array}$ & $\begin{array}{l}\text { Morlino } 1986 \\
\text { Morlino } 1986 \\
\text { Huntington } 1993^{317} \\
\text { Mezzetti } 2000^{318} \\
\text { Mezzetti } 2000 \\
\text { Karvonen \& Anckar 2001 } \\
\\
\text { Valenzuela } 1990^{321} \\
\text { Valenzuela } 1990\end{array}$ \\
\hline
\end{tabular}

313 Linz, J. J., Stepan, A. e Gunther, R., "Democratic Transition and Consolidation in Southern Europe, with Reflections on Latin America and Eastern Europe', in Gunther, R., Diamandouros, N. e Puhle, H. J. (a cura di), The Politics of Democratic Consolidation. Southern Europe in Compatative Perspective, op. cit., p. 81 y ss.

314 Spesso le medesime condizioni che hanno reso possibile la transizione si rivelano incapaci a risolvere gli ostacoli che si vengono a frapporre al consolidamento. Cfr. Crespo I., "Notas preliminares para el estudio de los procesos de consolidación democrática en América Latina", in: Revista Internacional de Sociología, No 12, maggio 1994.

315 Diamandouros, N., Puhle, H.-J. e Gunther, R., "Conclusion», in: Gunther R., Diamandouros N. e Puhle H.-J. (a cura di), The Politics of Democratic Consolidation. Southern Europe in Compatative Perspective, op. cit., pp. 389-412. 


\begin{tabular}{|c|c|c|}
\hline & FATTORI DICONSOLIDAMENTO & AUTORE DI RIFERIMENTO \\
\hline $\begin{array}{l}\text { economici } \\
\text { (strutturali) }\end{array}$ & $\begin{array}{l}\text { base sociale dell'autocrazia } \\
\text { forza della società civile } \\
\text { approfondimento della cittadinanza 5- } \\
\text { tipo di clearages 6- } \\
\text { valore della classe media } \\
\text { giustizia sociale/contenuta diseguaglianza } \\
\text { sociale } \\
\text { standard minimo di vita } \\
\text { standard educativo elevato } \\
\text { situazione psicosociale } \\
\text { impatto del cambiamento tecnico ed } \\
\text { economico-sociale } \\
\text { società civile pluralista } \\
\text { cultura politica } \\
\text { religione } \\
\text { grado di sviluppo economico } \\
\text { fattori economici } \\
\text { economia di mercato } 7 \text { - } \\
\text { democratizzazione della regione circostante } \\
\text { integrazione regionale (economica e/o politica) } \\
\text { cooperazione per la sicurezza } \\
\text { influenze internazionali } \\
\text { appoggio esterno ai programmi di } \\
\text { stabilizzazione }\end{array}$ & $\begin{array}{l}\text { Diamond } 2001 \\
\text { Diamond } 1998^{323} \\
\text { Mezzetti } 2000 \\
\text { Huntigton } 1993 \\
\text { Munck } 1994 \\
\text { Mezzetti } 2000 \\
\text { Morlino } 1986 \\
\text { Franklin Stevens } 2000^{324} \\
\text { Morlino } 1986 \\
\text { Llach } 1985^{8}\end{array}$ \\
\hline
\end{tabular}

316 Il modo in cui la transizione avviene è il principale determinante per il tipo di consolidamento prospettato. Cfr. Schmitter P. C., "The Consolidation of Political Democracies: Processes, Rhythms, Sequences and Typess, in: Pridham G. (a cura di), Transitions to Democracy. Comparative Perspectives from Southern Europe, Latin America and Eastern Europe, op. cit., pp. 535-569.

La transizione a mezzo di ruptura pare "una premessa migliore per il consolidamento". Cfr. Morlino L., Consolidamento democratico: alcune ipotesi esplicative, op. cit., pp. 439459.

"Una transizione pacifica [sarà] più utile al consolidamento democratico", mentre, tra le modalità, in ordine decrescente, favoriscono il consolidamento: transplacement, transformation, replacement. Cfr. Huntington S. P., The Third Wave. Democratization in the Late Twentieth Century, op. cit., pp. 276-277 (trad. it. La terza ondata. I processi di democratizzazione alla fine del XX secolo, op. cit., pp. 291-292).

317 Huntington, S. P., La terza ondata. I processi di democratizzazione alla fine del $X X$ secolo, op. cit., cap. V, par. 7.

318 Mezzetti, L., Le Democrazie Incerte. Transizioni costituzionali e consolidamento della democrazia in Europa orientale, Africa, America Latina, Asia, op. cit., pp. 571 581.

Sulla [soltanto] parziale affermazione della forma di Stato liberale, cfr. de Vergottini, G., Le transizizioni costituzionali. Sviluppi e crisi del costituzionalismo alla fine del XX secolo, Bologna, 1998, cap. V. 
1. Il grado di pluralismo accettato dal regime autocratico determina le prospettive di crescita per la società civile. Nella costituzione di una «memoria storica" non va tralasciata la violenza del regime autoritario contro la popolazione.

2. Esempio : esistenza di partiti di massa, sistema partitico, leadership capace, qualità delle élite, etc.

3. «Il consolidamento non è una semplice funzione del numero e della gravità dei problemi contestuali, ma del modo con cui le élite politiche e l'opinione pubblica rispndono all'incapacità dei nuovi governi e dei nuovi regimi di risolvere questi problemi». ${ }^{326}$

319 «Solo soluzioni istituzionali basate sul principio del consenso, piuttosto che su quello maggioritario, possono favorire il consolidamentom in: Morlino, L., Consolidamento democratico: alcune ipotesi esplicative, op. cit., pp. 439-459.

320 Karvonen, L. e Anckar, C., Party Systems and the Consolidation of Democracy: A Comparative Study of the Third World, XXIX Joint Sessions of Workshops, Workshop 13, Grenoble, aprile, 2001.

Per Mayorga, il moderate multiparty system è un fattore chiave : Mayorga R. A., Parliament arized Presidentialism, Moderate Multiparty System and State Transformation: The Case of Bolivia, Centro Boliviano de Estudios Multidisciplinarios (CEBEM), settembre, 1996.

321 Valenzuela, J. S., Democratic Consolidation in Post-Transitional Settings: Notion, Process, and Facilitating Conditions, Kellogg Institute, Working Paper No 150, dicembre, 1990 ; Field B. N., Pacting and the Consolidation of Democracy: The Spanish and Argentine Democracies in Comparative Perspective, American Political Science Association, Santa Barbara \& San Francisco-Towers, agosto-settembre, 2001.

Nella stessa direzione, Andersen M. E., Social Policy Dialogue: $A$ Window of Opportunity for Reform and Transformation in the 21st Century, Inter-American Development Bank, luglio, 2000, analizzando la Spagna post-franchista, in cui il patto della Moncloa servì ad implementare una politica economica non di scontro.

322 Spota, A. A., "Los aspectos psicosociales de la consolidación de la democracia argentina», in: AA. VV., "Sobre la Consolidación de la Democracia» [Jornadas sobre consolidación de la democracia, 20-21 settembre 1985], Buenos Aires, 1987, pp. 197-238.

323 Diamond, L., Political Culture and Democratic Consolidation, Instituto Juan March, Centro de Estudios Avanzados en Ciencias Sociales [CEACS], Working Paper No 118, giugno, 1998.

324 Stevens, F, Regional Integration and Democratic Consolidation in the Southern Cone of Latin America, ECPR, XXVIII Joint Sessions of Workshops, Workshop 18, Copenhagen, aprile, 2000.

325 Llach, J. J., "El apoyo externo a los programas de estabilización», in: AA. VV., «Sobre la Consolidación de la Democracia» Uornadas sobre consolidación de la democracia, 20-21 settembre 1985], Buenos Aires, 1987, pp. 165-173.

326 Huntington, S. P., La terza ondata. I processi di democratizzazione alla fine del XX secolo, op. cit., p. 292. 
Diamond ${ }^{327}$ ricollega ad alcuni precisi indicatori la bontà di una political performance: mantenimento dell'ordine politico, responsabilità, trasparenza, principio di legalità, legittimazione, contenimento dei livelli di criminalità, di corruzione politica, di politicizzazione del sistema giudiziario.

4. Rilevano fattori sociali ed economici. Spesso, infatti, la democrazia è confusa con la ricchezza, il benessere stesso (Hyman). ${ }^{328}$ Per Boussard, la diffusa povertà importa esclusione dal processo democratico, il che, a misura di tale esclusione, significa perdita di legittimità del sistema politico.

5. Vale a dire: progresso del diritto di cittadinanza dal formale all'esercizio e ampliamento delle dimensioni della cittadinanza dal civile al sociale.

6. Ridotte tensioni etnico-religiose, ad es., agevolano il consolidamento.

Per molte nuove democrazie è rilevante la dimensione di conflitto tra pro-democratici e anti-democratici. ${ }^{329}$

7. Whitehead dichiara come si sostenga, con poca autenticità, che l'unico percorso conducente al consolidamento democratico sia l'implementazione di riforme neo-liberal sul mercato, a tutto discapito dei valori della democrazia liberale. ${ }^{330}$

Con riferimento allo schema ${ }^{331}$ elaborato da Sschmitter relativo ai fattori che influenzano il tipo ed estensione del consolidamento, il cuore del

327 Diamond, L., "Future of Democracy», in: Clarke P. B. e Foweraker J. (a cura di), Encyclopedia of Democratic Thought, op. cit., pp. 154-161.

328 Hyman, G. F. (et. al.), Conducting a Democracy and Governance [DG] Assessment: A Framework for Strategy Development, Center for Democracy and Governance, U.S. Agency for International Development [USAID], Development Experience Clearinghouse [DEC], Document PN-ACG-505, Washington DC, dicembre, 1999.

329 Menéndez Moreno, A., Political Cleavages. Issues, Parties and the Consolidation of Democracy, Boulder-Oxford, 1999, in particolare le pp. 25 e 165. L'indicatore può riflettere l'estensione per cui la democrazia è considerata "the only game in town". L'Autore non manca di sottolineare come la frattura con lo scorrere del tempo e con il consolidarsi stesso della democrazia «è probabile [...] diventi meno centrale»: si tratta di una spaccatura transitoria, nel senso di temporanea (pagg. 19 e 24).

330 Whitehead, L., "The Alternatives to "Liberal Democracy": a Latin American Perspectiven, in: Held D., (a cura di), Prospects for Democracy. North, South, East, West, Cambridge, 1993, pp. 312-329. Lo scritto sembra essere stato frainteso da Joseph R., "Democratization in Africa after 1989: Comparative and Theoretical Perspectives", in: Anderson L. (a cura di), Transitions to Democracy, Columbia University, 1997.

331 Schema proposto da Schmitter, radicalmente rivisitato, come l'Autore invitava a fare. Cfr. Schmitter P. C., "The Consolidation of Political Democracies: Processes, Rhythms, 
modello è una serie di successive trasformazioni di strutture e modelli politici al livello di regime.

Variabili rubricate alle voci sono: eventi, scansione, trends e cicli. Individuare una variabile non comporta indicare un sicuro unico modo di influenzare il consolidamento: non si sa a priori se e come ciò avverrà. Il discorso vale anche per il più volte decantato trend dello «sviluppo economicom. ${ }^{332}$

Quanto a "virtù e fortuna", eufemisticamente chiamati altrove leadership o carisma, quale complemento delle capacità del leader individuale, va notato che tale concentrazione di potere non conduce al consolidamento delle istituzioni democratiche, anzi mina seriamente le strutture partitiche esistenti, le relazioni esecutivo-legislativo, il sistema di negoziazione degli interessi, etc.

Schmitter stesso esige che la classificazione non si abbia per assoluta, ma piuttosto rieditabile, con l'ipotetica perfettibile riallocazione di ogni voce.

In particolare rilevano velocità, cadenza e sequenza.

1. La velocità qui intesa è quella con cui i modelli/gli ideali divengono strutture.

La formazione di stabili e durevoli democrazie è dipinta come un processo lentissimo di graduale accrescimento e lento plasmare. I Paesi imprudenti e impazienti sarebbero condannati all'instabilità e al fallimento della democrazia. ${ }^{333}$ Il ritmo è talora accelerato e talora deviato da trends di varia natura.

2. La cadenza evidenzia come sia possibile individuare delle "epoche", momenti in cui il consolidamento democratico riguarda una serie di ordinamenti, appartenenti o meno ad una data regione geografica.

Sequences and Types", in: Pridham G. (a cura di), Transitions to Democracy. Comparative Perspectives from Southern Europe, Latin America and Eastern Europe, op. cit., p. 564.

332 Comunque il regime autoritario lascia tipicamente un "eredità" economica assai difficile, accidentata per i successori.

333 Esiste un secondo punto di vista, elaborato da Plumb (1973) : «l'accettazione da parte della società delle proprie istituzioni politiche e di chi le controlla può avvenire abbastanza rapidamente, quanto bruscamente l'acqua diviene ghiaccio». Il risultato può dipendere da fattori di lungo termine ("forze di marea"), ma non è così per le democrazie contemporanee. La realtà della stabilità è determinata dalle azioni di individui coscienti e calcolatori che creano nuovi arrangiamenti e organizzazioni in risposta a eventi e sfide abbastanza specifici. 
3. Infine, la sequenza. Le fasi di un cambiamento di regime (non solo per il consolidamento) si sovrappongono e non se ne può predire la durata. ${ }^{334}$ Alcune sequenze logiche sono inevitabili (ad esempio.: prima sessione delle camere, presentazione di progetti di riforma, iter di discussione ed approvazione legislativa, implementazione dell'innovazione) ; esistono poi quattro processi necessari e sei eventi solo eventuali. ${ }^{335}$

1. cambiamenti chiave [a livello di regime]

1.1. natura del regime democratico preesistente

1.2. natura del regime autoritario (con eventuale patto militare)

1.3. modo di dismissione del regime autoritario (con eventuale patto politico)

1.4. modo di transizione (con eventuale patto sociale)

2. sequenze

2.1. sequenze logiche

2.2. processi [necessari]

2.2.1. redazione o rivitalizzazione di una Costituzione

2.2.2. sottoposizione dei militari al controllo dei civili

2.2.3. formazione di un sistema partitico

2.2.3.1. modelli di voto

2.2.4. formazione di un sistema di gruppi di interesse

2.2.4.1. modelli di conflitto industriale

2.3. eventi [fatti che sono eventuali]

2.3.1. politiche con carattere di eventualità

2.3.1.1. formazione di un governo provvisorio

2.3.1.2. cambiamenti strutturali nella proprietà e ricchezza

2.3.1.3. revisione della distribuizione territoriale dell'autorità

2.3.2. patti [fatti che sono eventuali]

2.3.2.1. la negoziazione dell' uscita dei militari dal potere ("patto militare»)

2.3.2.2. la negoziazione di un patto tra partiti politici ("patto politico»)

2.3.2.3. la negoziazione di un patto sociale con la partecipazione dei gruppi d'interesse ("patto sociale»)

2.3.3. contingency [fatti eventuali che sono eventi inattesi]

2.3.3.1. intervento estero o occupazione

2.3.3.2. terrorismo, guerrilla

334 Schmitter riporta la boutade sulla fine del comunismo in Europa orientale : esso impiegò dieci anni in Polonia, dieci mesi in Ungheria, 10 giorni in Cecoslovacchia eaggiunge - 10 ore in Romania.

335 Ancora Schmitter critica la propria opera con O’Donnell (1986): «Abbiamo sottolineato il potenziale ruolo dei patti (politici, sociali, militari) per una pacifica e relativamente continua transizione dal regime autoritario, ma non abbiamo insistito sulla loro necessità per l'istaurazione o restaurazione della democrazia». 
2.3.3.3. cospirazioni militari

2.3.3.4. guerra

3. cadenza

3.1. livello e grado di diversificazione socioeconomica

3.2. conditionality [modo ed estensione dell'inserimento nel sistema internazionale]

3.2.1. norme internazionali

3.2.2. reti estere di sostegno

3.2.3. dipendenze

3.2.4. alleanze internazionali

3.2.5. esperienze simultanee a livello di regione

3.3. distribuzione e intensità delle spaccature etnico-linguistiche

4. trends [cd. "forze di marea"]

4.1. socioeconomici

4.2. onde lunghe

4.3. perturbazioni economiche cicliche

4.3.1. performance nel commercio internazionale

4.3.2. obsolescenza della produzione

4.3.3. debito estero

4.3.4. disoccupazione

4.3.5. fuga di capitali

5. imponderabile

5.1. virtù e fortuna

5.2. "destino"

\section{I luoghi del consolidamento democratico}

Alcuni Autori articolano la propria esposizione su piani progressivi. A ben vedere, si tratta di approfondimenti e precisazioni del termine "consolidamento", che vedono il concetto spostato dal sistema politico nazionale a sottosistemi o disaggregato in alcune dimensioni che gli pervengono. Spesso gli Autori indugiano sulle prime battute, delineano le traiettorie degli istituti in divenire, connettono la forma di un oggetto in più punti temporali determinati. 


\begin{tabular}{|c|c|c|c|c|}
\hline & $\begin{array}{l}\text { MORLINO: } \\
\text { AMBITI }\end{array}$ & $\begin{array}{l}\text { SCHMITTER: } \\
\text { LIVELLI }\end{array}$ & $\begin{array}{c}\text { LINZ \& STEPAN: } \\
\text { ARENE }\end{array}$ & $\begin{array}{l}\text { MERKEL: } \\
\text { LIVELLI }\end{array}$ \\
\hline 1 & $\begin{array}{l}\text { le strutture e le } \\
\text { procedure } \\
\text { democratiche }\end{array}$ & $\begin{array}{l}\text { Dimensione delle } \\
\text { singole istituzioni }\end{array}$ & $\begin{array}{l}\text { società civile (libera ed } \\
\text { attiva) }\end{array}$ & $\begin{array}{l}\text { Consolidamento } \\
\text { costituzionale }\end{array}$ \\
\hline 2 & $\begin{array}{l}\text { i rapporti } \\
\text { intrastrutturali }\end{array}$ & $\begin{array}{l}\text { Dimensione delle } \\
\text { interrelazioni tra } \\
\text { istituzioni }\end{array}$ & $\begin{array}{l}\text { società politica } \\
\text { (relativamente autonoma e } \\
\text { positivamente considerata) }\end{array}$ & $\begin{array}{l}\text { Consolidamento delle } \\
\text { strutture } \\
\text { rappresentative }\end{array}$ \\
\hline 3 & $\begin{array}{l}\text { la rappresentazione } \\
\text { territoriale degli } \\
\text { interessi }\end{array}$ & $\begin{array}{l}\text { Dimensione sesterna» } \\
\text { delle connessioni tra } \\
\text { istituzioni e società } \\
\text { civile }\end{array}$ & nde of lawe & $\begin{array}{l}\text { Consolidamento } \\
\text { comportamentale delle } \\
\text { tetodite }\end{array}$ \\
\hline 4 & $\begin{array}{l}\text { la rappresentazione } \\
\text { funzionale degli } \\
\text { interessi }\end{array}$ & Dimensione egemonica & apparato statale & $\begin{array}{l}\text { Consolidamento della } \\
\text { cultura civica }\end{array}$ \\
\hline 5 & $\begin{array}{l}\text { i rapporti tra strutture } \\
\text { di intermediazione e } \\
\text { società civile }\end{array}$ & & $\begin{array}{l}\text { società economica } \\
\text { istituzionalizzata }\end{array}$ & \\
\hline 6 & $\begin{array}{l}\text { i rapporti tra strutture } \\
\text { di intermediazione e } \\
\text { regime }\end{array}$ & & & \\
\hline 7 & $\begin{array}{l}\text { i rapporti tra società } \\
\text { civile e regime non } \\
\text { mediati da alcuna } \\
\text { struttura }\end{array}$ & & & \\
\hline
\end{tabular}

\subsection{Gli ambiti nell'elaborazione di Morlino}

Il consolidamento democratico si manifesta in diverse direzioni, attinenti a certi ambiti. ${ }^{336}$ Leonardo Morlino sottintende una pretesa esaustività, giacchè indica compiuto il sistema in 6 categorie (a-f), non attribuendo rilevanza alcuna alla settima $(\mathrm{g})$.

\section{a. le strutture e le procedure democratiche}

Le modalità che vengono in rilievo sono le seguenti : emergono prassi decisionali; si perfezionano i meccanismi di esecuzione delle decisioni; si affina il controllo delle risorse, affinchè le decisioni siano non solo eseguite ma anche obbedite; si razionalizzano le strutture amministrative; si adatta l'ordinamento alle necessità di funzionamento del regime; si rivisita la normativa pre-democratica; si cementano interessi al mantenimento delle istituzioni;

336 Morlino, L., Consolidamento democratico: definizione e modelli, op. cit., pp. 210-216. 
b. i rapporti intrastrutturali

Sono particolarmente importanti i rapporti tra i poteri costituiti.

Modalità: emergono prassi di comportamento e si fissano i ruoli; si aggiusta il modello costituzionale quanto alle relazioni tra strutture; si controlla e si mantiene entro il quadro democratico il conflitto; si contengono gli interessi delle agenzie pubbliche entro confini legali e in particolare si escludono dall'arena coercitiva i militari;

c. la rappresentazione territoriale degli interessi

Modalità: si rafforza l'organizzazione dei partiti; se ne determinano la identità e le immagini, mentre si configura un elettorato stabile; si fissano logiche competitive all'interno del sistema partitico;

d. la rappresentazione funzionale degli interessi

Modalità: le strutture di interesse assumono identità, rafforzano la propria organizzazione ed esprimono gli interessi; stabiliscono canali e modalità di comunicazione e di pressione;

e . i rapporti tra strutture di intermediazione e società civile

Modalità: acquisiscono identità ed autonomia le strutture intermedie;

f. i rapporti tra strutture di intermediazione e regime

I fenomeni rientranti in tale ambito sono la base del consolidamento e in quanto tali toccano ogni altro ambito. Modalità: le strutture di intermediazione acquisiscono legittimazione e maggiore conseguente integrazione, fino a privare il tessuto democratico di sfide interne;

g. i rapporti tra società civile e regime non mediati da alcuna struttura

Modalità: si tende al contenimento di atteggiamenti diffusi di insoddisfazione, protesta, alienazione, passività e indifferenza. Morlino ritiene questo un ambito inesistente, essendo comunque necessario l'intervento governativo e delle strutture.

\subsection{I livelli nella concezione di Schmitter}

Accettare la nozione di regimi parziali di Schmitter significa staccarsi dalla presunzione che l'intero insieme di regole istituzionali che costituisce un regime si trovi istallato in un singolo momento temporale (Munck). ${ }^{337}$ 
Schmitter ${ }^{338}$ distingue tra consolidamento della democrazia e consolidamento degli ordinamenti [o «regimi»] parziali (inteso, quest'ultimo, quale l'emergenza di stabili istituzioni e modelli di interazione tra le rispettive sfere).

L'Autore $^{339}$ indica tre piani su cui il consolidamento democratico si concreta.

1) Dimensione delle singole istituzioni.

Vengono in considerazione i processi di acquisizione di identità ed autonomia e quelli di strutturazione dei "gruppi».

2a) Dimensione dell'interrelazioni tra istituzioni.

Schmitter parla di strutturazione di «regimi parziali», in cui due o più tipi di gruppi confluiscono e, concorrendo e cooperando, definiscono meta-regole $\mathrm{e}$ istituzioni che rendono $\mathrm{i}$ loro reciproci comportamenti più prevedibili. ${ }^{340}$

I «regimi [interni] parziali» indicati da Schmitter sono sei:

a. il regime costituzionale. Ha come fulcro l'Esecutivo, che intrattiene relazioni con quattro complessi istituzionali: l'assemblea legislativa, i partiti politici, le agenzie amministrative e i gruppi d'interesse;

b. il regime della concertazione. Riguarda i gruppi d'interesse e i meccanismi di negoziazione e implementazione politica in cui questi interagiscono direttamente con le agencies amministrative;

c. il regime della pressione. Attiene ai gruppi sociali che si pongono tra gruppi d'interesse e Parlamento;

337 Munck, G. L., Disaggregating Political Regime: Conceptual Issues in the Study of Democratization, Kellogg Institute, Working Paper No 228, New Haven, agosto, 1996.

338 Schmitter, P. C., "Organized Interests and Demcoratic Consolidation in Southern Europen, in: Gunther R., Diamandouros N. e Puhle H.-J. (a cura di), The Politics of Democratic Consolidation. Southern Europe in Compatative Perspective, op. cit., pp. 284-314.

339 Schmitter, P. C., "The Consolidation of Political Democracies: Processes, Rhythms, Sequences and Typess, in: Pridham G. (a cura di), Transitions to Democracy. Comparative Perspectives from Southern Europe, Latin America and Eastern Europe, op. cit., pp. 533-569, in particolare pp. 551-559.

340 I regimi parziali emergono per convertire eccezionali contingenze in standard routines, dichiarazioni di principio in regole di prudenza, patti temporanei in stabili compromessi, in sostanza modelli in strutture. Solitamente ciò accade mentre si redige e ratifica una nuova Costituzione. Se l'elettoralismo è la panacea della transizione, il costituzionalismo è la panacea del consolidamento democratico. 
d. il regime della rappresentanza. S'instaura tra Parlamento (e, più in generale, ogni carica e fonte d'influenza), da un lato, e partiti, gruppi e movimenti dall'altro;

e. il regime elettorale. Riguarda Parlamento e partiti in via principale. In particolare, prendono nitida forma l'ideologia, l'organizzazione e l'immagine dei partiti, nonché i codici elettorali;

f. il regime clientelistico. Riguarda i partiti politici.

2b) Dimensione «esterna» delle connessioni tra istituzioni e società civile.

Dal funzionamento delle relazioni, per lo più informali e sociologiche, tra «regimi parziali» e regimi esterni dipende il perdurare della legittimazione [del regime]. Il punto riguarda quanto le istituzioni siano accessibili, forniscano soddisfacente partecipazione e assicurino rispondenza alle preferenze dei cittadini.

3) Dimensione egemonica.

In una prospettiva positiva, è necessario il radicarsi del regime emergente nelle pratiche e norme sociali di ogni giorno. La Gestalt delle istituzioni emergenti è relazionata ai gruppi sociali ed economici il cui supporto è cruciale per la sopravvivenza nel lungo periodo delle istituzioni. Queste devono, in altre parole, convincere i propri cittadini che i meccanismi di rappresentazione e decisione esistenti sono propriamente (se non perfettamente) democratici.

In una prospettiva negativa, si può sostenere avvenuta la strutturazione egemonica quando certe forme di violenza, resistenza o lotta non appaiono. ${ }^{341}$

\subsection{Le cinque arene di Linz e Stepan}

Linz e Stepan ${ }^{342}$ circoscrivono cinque «arene» perché una democrazia possa consolidarsi, ciascuna delle quali imperniata su un principio [normativo] fondante. Esse sono:

341 Espressa in termini positivi questa seconda condizione risulta un'endiadi: «i protagonisti dotati di risorse competono secondo le regole», mentre "i subordinati disobbediscono senza scontri", accettando il compromesso per cui ad alcuni è garantito il diritto di dettare le politiche e ricoprire gli incarichi, per altri sono garantiti determinati benefici.

342 Linz, J. J. e Stepan, A., Problems of Democratic Transition and Consolidation. Southern Europe, South America, and Post-Communist Europe, op. cit. 
1. una società civile (parte dell'arena politica in cui gruppi e individui cercano di articolare valori, creare legami e promuovere i propri interessi) libera ed attiva; ${ }^{343}$

2. una società politica relativamente autonoma e considerata favorevolmente, in cui il sistema politico si strutturi specificamente per rivendicare il legittimo diritto di esercitare il controllo sui poteri pubblici e sull'apparato statale [competizione elettorale libera e inclusiva];

3. rule of law; rispetto per le leggi, ovvero una rule by law che assicuri garanzie legali per le libertà [costituzionalismo];

4. apparato statale : una burocrazia statale che possa esser impiegata dal nuovo governo democratico. Il governo democratico deve essere in grado di esercitare effettivamente la sua pretesa di detenere il monopolio dell' uso legittimo della forza, e le democrazia moderna richiede un'effettiva capacità di controllo, regolazione ed estrazione delle risorse. Pertanto richiede una burocrazia funzionante ed efficace [norme burocratiche razionali-legali $] ;^{344}$

5. una società economica istituzionalizzata: l'economia controllata dallo Stato non coesiste con la democrazia, empiricamente; nè esiste una democrazia consolidata in presenza di una perfetta economia di mercato. [mercato istituzionalizzato].

Si parla altresì di "economia politica della legittimità»: a parità di altri fattori, il Paese che presenta una crescita economica positiva ha maggiori probabilità di consolidare la democrazia rispetto ad un Paese che presenta invece una crescita negativa. Il consolidamento è ostacolato e persino la democrazia de-consolidata qualora gli attori chiave si rassegnino ad una democrazia caratterizzata da un basso livello di performance economica, poichè le alternative non sono appetibili.

Le critiche al sistema aggraveranno le ripercussioni generate da periodi di difficoltà economica, se i cittadini si persuadono che il sistema democratico in quanto tale stia trascurando il problema economico o sia incapace di implementare serie riforme economiche. Si sconfina nel crollo della democrazia qualora potenti gruppi (anche esterni) si convincano

343 Successivamente gli attori operano una stretta connessione con l'arena sub 3- [rule oflaw], distinguendo in quest' ultima il costituzionalismo. Vedi tavola 4.3.

344 Vedi Suleiman E., «Bureaucracy and Democratic Consolidation: Lessons from Eastern Europen, in: Anderson, L. (a cura di), Transitions to Democracy, Columbia University, 1997. 
sempre più che le alternative di governo non democratiche siano l'unica soluzione per risolvere la crisi economica.

\subsection{I livelli di Merkel}

La ricostruzione di Wolfgang Merkel vorrebbe indicare le prospettive di consolidamento per l'intero sistema politico. ${ }^{345} \mathrm{Il}$ progresso da un livello al seguente appare, però, determinato dalle sole élite.

\section{Consolidamento costituzionale.}

Il vero consolidamento della democrazia comincia solo quando è stata redatta una nuova Costituzione, Carta che stabilisca le procedure costituzionali di interazione politica ( rules of the game») e auspicabilmente parta da un raggiunto accordo sui valori democratici fondamentali ( $v$ value consensus") o, meglio ancora, da un programmatic consensus.

\section{Consolidamento delle strutture rappresentative.}

Concerne la rappresentazione d'interessi a livello territoriale e funzionale.

Tre sono le condizioni più importanti relativamente a partiti e gruppi:

- essi devono compiere il proprio ruolo in nome e per conto della popolazione, il che implica che essi debbano essere saldamente radicati nella società;

- essi devono conformarsi alle regole democratiche e perseguire politiche non anticostituzionali, nè intraprendere attività sleali o parzialmente leali;

- la loro struttura interna e i loro processi decisionali devono osservare uno standard democratico minimo.

Giacchè non possono rimuovere eventuali deficit democratici, strutture rappresentative disfunzionali hanno un impatto fatale sul consolidamento, conducendo a forme di "defective democracy». ${ }^{346}$

\section{Consolidamento comportamentale delle veto-élite}

Vi sono élite informali la cui posizione influente ne fa degli attori politici rilevanti, pur situandosi al di fuori del sistema politico tradizionale.

345 Merkel, W., "Institutionalisierung und Konsolidierung der Demokratien in Ostmitteleuropa», in: Merkel W., Sandschneider E. e Segert D., (a cura di), Systemwechseh, Tomo 2, Die Institutionalisierung der Demokratie, Opladen, 1996, pp. 73-112.

346 Merkel, W., Defective democracies, Instituto Juan March, Centro de Estudios Avanzados en Ciencias Sociales [CEACS], Working Paper No 132, marzo 1999, successivamente in: Systemtransformation, Opladen, 1999. 
Esemplificando: i militari, l'aristocrazia terriera, finanzieri, imprenditori e leader politici radicali. Di esse si parla come di veto-élite poichè è in loro potere sostenere, minare e persino rovesciare la democrazia, mediante il proprio comportamento.

4. Consolidamento della cultura civica.

Completati i primi tre livelli - a carico delle élite - la democrazia diviene "sustainable». Paradossalmente, il demos, cui la democrazia deve il nome, contribuisce assai poco al consolidamento: le masse rivestono un ruolo insignificante, trascurabile all'inizio del consolidamento.

Riemerge il ruolo delle masse, allorchè il sostegno civile si rivela essere cruciale per la stabilità a lungo termine del regime. L'endiadi che ne consegue è tra atteggiamenti («civic culture») e azioni («civil society»).

\section{Conclusioni}

Non sono mancati, anche in tempi recenti, vari tentativi nel senso della identificazione di una nozione minimale della democrazia alla luce della molteplicità delle variabili in precedenza analizzate. D. Collier e $S$. Levitsky, ad esempio, hanno potuto ricostruire ben 550 sottotipi di democrazia rinvenibili in seno ai 150 studi e saggi dottrinali che più recentemente si sono incentrati sul tema: $:{ }^{347}$ molti dei sottotipi menzionati, tuttavia, si limitano a porre in evidenza specifiche caratteristiche istituzionali ovvero tipi di democrazie mature, mentre altri denotano forme minori e menomate di democrazia. Questi ultimi, in particolare, sono soggetti a grandi variazioni secondo la enfasi terminologica e concettuale che viene utilizzata in sede di teorizzazione. Il ricorso ad una aggettivazione capace di evocare una caratterizzazione ed un profilo di ordine sociale ed economico -approccio ritenuto particolarmente attraente negli anni sessanta e settanta- ha conosciuto un costante declino negli ultimi due decenni del XX secolo. Le moderne concezioni della democrazia appaiono piuttosto caratterizzate da divergenze in merito alla entità ed alle dimensioni, qualitative e quantitative, dei sistemi democratici, e quanto alla loro qualificazione sulla base di criteri di tempo e di spazio, che si intersecano

347 Cfr. Collier D. - Levitsky S., "Democracy with Adjectives : Conceptual Innovation in Comparative Research", in: World Politics, 1997, p. 430 y s. 
con coordinate di natura politica, storica, filosofica, religiosa, economica, antropologica.

Concezioni minimaliste della democrazia ne identificano la essenza, come si è osservato, nella competizione elettorale quale indefettibile meccanismo capace di favorire un fisiologico processo politico decisionale. ${ }^{348}$

Tali concezioni hanno peraltro implicato, come parimenti si è osservato, la elaborazione e il riconoscimento di requisiti ulteriori al fine della qualificazione in senso pienamente ed effettivamente democratico di singole esperienze costituzionali : il contenuto semantico del concetto di poliarchia rinvenibile nella ricostruzione di $\mathrm{R}$. Dahl poggia propriamente sulla esistenza di condizioni quali la presenza, accanto a rappresentanti eletti e ad elezioni libere, eque e frequenti, di partecipazione effettiva, parità di voto, libertà di espressione, fonti alternative di informazione, diritto all'informazione, autonomia associativa, cittadinanza allargata, universalità del suffragio. ${ }^{349}$

La categoria delle democrazie elettorali che è così venuta emergendo sembra tuttavia rappresentare unicamente un tipo o una categoria classificatoria intermedia rispetto al continuum descritto, ad un livello superiore, dalle democrazie liberali e, ai livelli inferiori, dalle pseudodemocrazie e dalle non democrazie.

$\mathrm{La}$ democrazia liberale, in particolare, appare elevarsi, rispetto alle concezioni meramente o prevalentemente formali in precedenza menzionate, per la necessaria presenza di requisiti ulteriori che, sulla scia della ricostruzione di Diamond più volte richiamata, ${ }^{350}$ possono compendiarsi nei seguenti fattori od elementi sostanziali : rappresentanti eletti ; fisiologico funzionamento del principio della separazione dei poteri, sia in senso verticale che orizzontale (horizontal accountability), con particolare riferimento alla indipendenza del potere giudiziario ; fisiologico funzionamento del principio di separazione fra potere civile e potere militare e garanzia della prevalenza del primo sul secondo ; adozione del principio della separazione fra Stato e religione ; effettiva garanzia dei diritti dell'opposizione politica e parlamentare in particolare ; garanzie a favore delle minoranze culturali, etniche, religiose; garanzia dei diritti di

348 Cfr. Schumpeter, J., Capitalism, Socialism, and Democracy, Harper, New York, 1947 , p. 269.

349 Cfr. Dahl, R., Sulla democrazia, op. cit., pp. 98-99.

350 Diamond, L., Developing Democracy. Towards Consolidatio, op. cit., pp. 11-12. 
espressione e di rappresentanza degli interessi dei cittadini, nonché del diritto di associazione; presenza di fonti alternative di informazione e garanzia del diritto di accesso alle medesime; riconoscimento e garanzia effettiva del principio di eguaglianza; riconoscimento e garanzia effettiva dei diritti fondamentali della persona.

Tali condizioni implicano tuttavia un pre-requisito: la condivisione, in seno alla singola esperienza costituzionale considerata, della Costituzione quale comune e generale tavola di valori, prescindendosi quindi dal loro contenuto assiologico, che può assumere una diversa connotazione nell'ambito delle varie forme di Stato o in seno a contesti geografici e culturali diversi: l'assenza dello spirito della Costituzione e della lealtà verso la Costituzione, vale a dire della concezione della Costituzione quale strumento fondamentale atto a garantire la stabilità e la continuità dell'ordinamento incentrato su principi basilari intimamente accettati e condivisi da parte dei membri della società che è destinata a reggere, sembra configurarsi come una delle principali cause di fragilità e debolezza delle democrazie illiberali venute ad esistenza nel contesto della terza ondata dei processi di democratizzazione che ha interessato il mondo postcomunista e gli ordinamenti del terzo mondo.

In assenza (o in conseguenza del venire meno) di uno o più dei requisiti citati, può assistersi ad uno slittamento delle democrazie liberali verso la categoria delle democrazie elettorali ovvero ad una involuzione delle democrazie elettorali in senso pseudodemocratico o semidemocratico o finanche autoritario: lo stato patologico che può aggredire i singoli sistemi democratici che attraversino esperienze di transizione costituzionale e sperimentino percorsi di consolidamento democratico sembra potersi concretare in una stagnazione del tasso qualitativo e quantitativo della democrazia ovvero in un regresso dato dalla fluttuazione all'interno di categoria strutturalmente e concettualmente subordinata.

I fattori capaci di rendere particolarmente vischioso e poroso il processo di evoluzione o di stagnazione democratica ovvero di implicare la qualificazione di singole esperienze quali democrazie vane o vacue (hollow democracies) sono riconducibili, come si è visto, a matrici diversificate, quali l'assetto istituzionale e costituzionale, i sistemi elettorali e partitici, il ruolo dei militari, l'incidenza di concezioni religiose e filosofiche, il contesto economico e strutturale, la struttura della società civile, la natura dei processi di transizione costituzionale, l'esito degli esperimenti di innesto costituzionale e di consolidamento democratico. 



\section{Transiciones constitucionales y consolidación de la democracia en los albores del siglo XXI}

Luca Mezzetti*

En los albores del siglo XXI se debe reconocer, al constitucionalismo liberal, un éxito múltiple sobre diversos aspectos. En efecto, por una parte, el modelo liberal ha cumplido en modo pleno (o está a punto de cumplir) la misión histórica que se le ha confiado con el fin de lograr una consolidación definitiva de la democracia en un número de ordenamientos que no es irrelevante, que coincide con las experiencias constitucionales de Europa occidental y de los países de matriz anglosajona del área norteamericana (Estados Unidos y Canadá) y del área pacífica (Australia y Nueva Zelanda), incluyendo también en esta última el caso de Japón. Se puede decir que el éxito del constitucionalismo liberal, en los ordenamientos mencionados, es pleno.

De todas formas, por otra parte, no se puede dejar de observar que el modelo liberal haya representado, en el contexto de las diversas oleadas de democratización que han tenido lugar después del año 1945, un punto de referencia para un número elevado de ordenamientos afectados por el proceso de descolonización y, en modo más reciente, afectados por el cambio de la forma de estado y de gobierno debido a la caída de los regímenes comunistas (es el caso de los países de Europa centro-oriental y del área ex-soviética) o bien, afectados por el tentativo de democratización y modernización de las estructuras constitucionales y de los sistemas políticos que proceden de una experiencia post-colonial (es el caso de los países africanos y asiáticos). En este segundo grupo de ordenamientos, el éxito del constitucionalismo liberal se evidencia en forma parcial.

\footnotetext{
"Traducción resumida a cargo de Claudia Herrera.
} 
En realidad, con relación a las funciones y a las tendencias del modelo constitucional liberal, se puede identificar un denominador común en los dos grupos anteriormente mencionados: la capacidad del modelo mismo de actuar como instrumento de resistencia y de reacción en las fases de deslegitimación política de los mitos antidemocráticos (Habermas), e igualmente como instrumento de consolidación de las conquistas democráticas en las fases sucesivas. En las experiencias constitucionales del mundo occidental, tales fases coincidieron por una parte, con la derrota de los regímenes autoritarios en 1945, y por otra, con la creación del Estado social de derecho; en las experiencias constitucionales de los países recién independizados (o que la hayan readquirido) o en vía de desarrollo (incluyendo en esta categoría a los ordenamientos de Europa centro-oriental), con la descolonización y con el comienzo de la modernización del orden institucional y económico, o bien al refundar el mismo, en el caso de los ordenamientos ex-socialistas. En tal sentido el «siglo breve», expuesto por la doctrina prestigiosa del historicismo (Hobsbawm) como trayectoria que se despliega sin solución de continuidad desde 1914 a 1989 , se manifiesta en realidad caracterizada por una cesura profunda y positiva - la victoria de las democracias contra las autocracias - capaz de configurarse como acontecimiento que puede privar de «cualquier legitimación quien desde entonces no hubiera rendido homenaje (si bien solo de manera oral, si bien solo con referencia a la forma literal) al espíritu universal de la ilustración políticas (Habermas).

La difusión parcial que el constitucionalismo liberal ha registrado en el contexto europeo-oriental, africano, latinoamericano y asiático se puede explicar por una serie de razones, que no se atribuyen de manera restrictiva a la dificultad de implantar y trasladar el modelo liberal en el seno de los ordenamientos que se encuentren preparados para acoger algunos contenidos y principios esenciales, rechazando - por el contrario- a otros. En efecto, al lado de los motivos o las causas de orden jurídico y cultural, surgen otras de tipo económico y estructural; o que derivan de factores de matriz exógena y endógena; o que proceden de las diversas peculiaridades de la sociedad civil; o que se identifican con razones de tipo religioso; o que son atribuibles a la función de los militares y de las fuerzas armadas. A la luz de tales consideraciones se puede comprender el hecho que un gran número de ordenamientos todavía sea llamado a medirse con un desafío doble: la solución de los problemas de desarrollo, la modernización de la propia estructura institucional y los asuntos generados a raíz de la globalización y de la internacionalización. 
En un gran número de hipótesis, las tareas conexas a estos asuntos con frecuencia se han mostrado (y se muestran todavía), como una carga de alcance cualitativo y cuantitativo excesivamente onerosa para las gráciles estructuras de los ordenamientos que, con fatiga una vez superada la fase de transición constitucional, se hallan en la fase de consolidación democrática que no es menos delicada de afrontar. En tal sentido, resulta de particular interés evocar, la imagen delineada por la acreditada doctrina política de Huntington, para subrayar la naturaleza (al menos de manera potencial) anfidroma de los procesos de democratización: a cada oleada de democratizaciones - que representa para algunas experiencias constitucionales el momento de atraco de una nave después de una navegación peligrosa en las aguas de la transición institucional - corresponde una oleada de regreso (reverse wave) que arrastra a otras, extenuadas y agotadas en el intento por llevar a cabo tal tarea doble (enorme), hacia el limbo, jurídico y político, de las democracias inciertas o hacia el abismo del regreso autoritario.

Por lo tanto, surge como una tarea fundamental de la doctrina constitucional, frente al actual y difuso interés por los temas de globalización y de la necesidad de suministrar respuestas adecuadas a los desafios planteados al constitucionalismo en el contexto internacional en su complejo (definición de funciones nuevas y actualización de las funciones del constitucionalismo; reformulación del ordenamiento y de las funciones del Estado nacional; definición de nuevos y diversos alcances del concepto y el contenido de la soberanía popular), preguntarse sobre la funcionalidad del constitucionalismo durante la consolidación democrática en un gran número de ordenamientos y de experiencias constitucionales que se hallan todavía a mitad del paso de la misma transición y que muestran índices de incertidumbre y de no cumplimiento en cuanto a la realización plena de la acción de convergencia entre el dato constitucional formal y la garantía de efectividad de los principios y de los instrumentos codificados en su seno.

Afrontar los problemas mencionados significa revelar los paralelismos y las asimetrías en el ámbito de los itinerarios seguidos por los diferentes conjuntos de ordenamientos durante la evolución y/o regresión de los procesos de transición constitucional y de consolidación de la democracia, tratando de delinear una serie de factores que, en cuanto dotados de una aptitud positiva o negativa, se han revelado en grado, respectivamente, según un nivel de gravedad decreciente; de impedir el proceso de tran- 
sición hacia la democracia y el pasaje sucesivo a la fase de consolidación institucional; de volver al proceso mismo extremadamente viscoso y particularmente incierto en sus éxitos finales de la fase de consolidación democrática. En fin, de influir según un grado de intensidad diverso, sobre el cumplimiento de la segunda fase y, en particular, sobre el éxito de las intervenciones de la ingeniería constitucional y de la reforma estructural realizadas al interior de cada uno de los ordenamientos.

Prescindiendo de los factores exógenos, de orden internacional o regional, los factores endógenos capaces de condicionar el perfeccionamiento de la fase de transición constitucional, y además los requisitos para consolidar la democracia, son atribuibles de manera esencial a cuatro contextos de referencia (relativos en forma respectiva); a la naturaleza del proceso de transición constitucional; al carácter del sistema económico adoptado por el ordenamiento en particular; a la cultura política del mismo; en fin al tipo de estructura constitucional escogida de manera.

\section{Las perspectivas de consolidación democrática resultan por lo ge-} neral condicionadas por la naturaleza del régimen anteriormente vigente

Aunque no resulta posible configurar una relación causa-efecto que sea mecánica y automática, entre la forma del régimen precedente y la forma de estado y de gobierno en vía de instauración-consolidación (Beetham D., "Conditions for Democratic Consolidation", en: Review of African Political Economy, 1994, p. 162), por una parte, es plausible en modo intuitivo, que una experiencia democrática previa haya dejado sedimentos positivos para la sostenibilidad de la democrazia por el pueblo y constituya una oportunidad válida con el objeto de no repetir los errores o causar las desviaciones ocurridas en el pasado; por otra parte, es posible que se verifiquen una serie de turbulencias durante el proceso de democratización, que tienden a traducirse, en algunos casos significativos, en actos de intento por debilitar el proceso mismo en sus fundamentos (es el caso de Rusia durante el período que sigue al año 1990), o bien se genera una alternancia entre períodos de gobierno democrático y períodos de gobierno autoritario (es el caso del "péndulo» que ha caracterizado a la mayoría de los ordenamientos del continente latinoamericano, al menos hasta la mitad de los años ochenta), o provoquen una sensación de des- 
afección y de escepticismo en cuanto a las perspectivas de consolidación democrática a largo plazo.

Si bien no es posible, de manera general, instaurar una conexión sistemática entre el tipo de régimen precedente y las perspectivas democráticas futuras, dos categorías de regímenes diferentes se han revelado en grado de dejar a sus sucesores una herencia particularmente pesada y penetrante, capaz de condicionar de manera profunda las trayectorias evolutivas y los contenidos de las reformas que se adopten por parte del nuevo régimen: regímenes militares y regímenes comunistas.

En particular, la superación de los regímenes militares implica la difícil tarea de despolitizar las fuerzas armadas y su propia organización con el fin de impedir en un futuro la intervención en política y de definir las esferas de separación entre el poder civil y el poder militar. Sin embargo sobre la base de la subordinación del segundo al primero como supuesto imprescindible. Tal tarea resulta más fácil cuando el régimen militar que se pretende superar termina por ser desacreditado o cae a consecuencia de una derrota militar (son los casos de Grecia, después de 1975, y de Argentina, después de 1983); se vuelve más complejo e incierto en su éxito final cuando el gobierno militar negocia a su propio favor un papel garantizado o un poder de veto frente al sucesor democrático (es el caso chileno posterior a 1990).

Por otra parte, el ocaso de los regímenes comunistas ha implicado la tarea - de enormes proporciones - de introducir en modo paralelo y simultáneo una economía de mercado y estructuras e instituciones democráticas. La simultaneidad descrita ha enseñado en múltiples casos específicos (en tal sentido constituyen un caso emblemático las experiencias de los países de Europa centro-oriental) los peligros inherentes en la operación de amplia escala consistente en la introducción de un nuevo sistema estructural (de mercado) en lugar de una economía planificada y de un nuevo ordenamiento institucional (democrático) en lugar de un ordenamiento autocrático-burocrático-centralista. Tales peligros residen, de manera particular, en los "costos" sociales y humanos que tal obra de restructuración implica y en el consecuente escepticismo destinado a apoderarse de los sujetos afectados por este cambio doble: esto ha generado, como consecuencia extrema, unos reflujos políticos que se han traducido, en algunos casos significativos (países Bálticos, Polonia, Bulgaria, Hungría), en el regreso al poder, aunque sea momentáneo, de la clase política socialista (depurada y reciclada), que de todos modos resulta dotada en 
los casos mencionados de un notable poder de negociación, de los contenidos de las reformas y de los tiempos y de las modalidades de realización de las mismas, con las fuerzas políticas que han desarrollado un papel crucial en la fase de transición constitucional.

En estas hipótesis el proceso de consolidación democrático inevitablemente se vuelve más lento y articulado y aparece menos evidente la solución de continuidad. Por el contrario, en otra parte más marcada y profunda, que separa el régimen pre-democrático del democrático.

\section{Las modalidades de transición hacia la democracia ejercen influen-} cia en la consolidación sucesiva

La doctrina ha experimentado por mucho tiempo y en modo diverso múltiples intentos para elaborar las tipologías de los procesos de transición, que no son fáciles de sintetizar.

Por ejemplo, es de recordar, como Huntington (en su ensayo "Democracy's third wave", en: Journal of Democracy, 1991, p. 12 y ss.) identifica un proceso que combina transformación (transformation), ya iniciado en el ámbito de un régimen autoritario, y sustitución (replacement), puesto en marcha por la sociedad civil y por la oposición, que el autor define con un neologismo como transplacement. Del mismo modo encontramos otra tentativa de síntesis conceptual en la obra de Linz ("Transitions to Democracy", en: The Washington Quarterly, 1990, p. 143 y ss) en la cual combina reforma (reform), proceso de cambio gradual y negociado, y ruptura (rupture), proceso de cambio de naturaleza rápida y radical, identificando una trayectoria evolutiva de tipo transactivo (transaction). En realidad estas y otras categorías son susceptibles de un gran número (tal vez infinito) de entrelazamientos y sus diferentes intersecciones permiten formular la hipótesis de varias tipologías, todas adherentes en cierta medida a experiencias concretas y no obstante desprovistas de una capacidad plena para suministrar una explicación satisfactorias de los procesos de transición constitucional y de consolidación democrática que han afectado las experiencias mismas.

En este sentido, es probable que resulte más conveniente la utilización, al lado de tales categorías tipológicas y de manera subsidiaria -que no significa residual - de otro criterio para identificar la naturaleza de los procesos de democratización: el alcance cualitativo y cuantitativo del consenso 
que acompaña a los procesos mismos, su difusión y capilaridad en el seno de la colectividad y de los grupos de interés que la caracterizan, así como la identidad de los sujetos protagonistas de los procesos de transición.

Por otra parte, la función de tal criterio ha sido asumida de manera implícita por la doctrina que, con respecto a la naturaleza incluyente o excluyente de los procesos de transición, ha desarrollado la teoría de los «pactos entre las élites" (ver O'Donnell-Schmitter-Whitehead, Transitions from Authoritarian Rule, Baltimore, 1986, passim; Przeworski, Democracy and the Market, Cambridge, 1991, passim) y ha subrayado que las perspectivas futuras de consolidación de la democracia sean incrementadas no solo a partir de un acuerdo formal sobre reglas de competición política entre los diferentes sectores de la élite política, sino también a partir de acuerdos informales que se estipulen con el fin de limitar la agenda de competición política, en modo tal que ninguno de los grupos se sienta amenazado en sus propios intereses que perciben como vitales.

La difusión del consenso representa una ventaja clara para la consolidación democrática. Sin embargo, los "pactos entre las élites» pueden resultar vulnerables bajo dos aspectos bien diversos. En efecto, si hay la participación de fuerzas que en modo irreducible resultan ser de naturaleza antidemocrática, por ejemplo los militares, en este evento las modalidades de transición pueden convertirse en objeto de compromisos bien amplios. De otra parte, si los pactos mismos producen consenso, por lo menos temporal y de manera transitoria, excluyendo a las instancias populares o a las fuerzas populares (hipótesis de «democracia a través de medios no democráticos» - democracy through undemocratic jeansenunciada por O'Donnell), resultarán vulnerables frente al planteamiento de tales instancias en un futuro.

Dicho de otro modo, es oportuno tomar en consideración - como se había mencionado con antelación- el criterio de profundidad y de difusión y extensión del proceso de transición y del consenso que lo acompaña, es decir en que medida este se introduce en la sociedad y no solo en las élites políticas. En tal sentido se yuxtaponen dos casos paradigmáticos, que es del caso aducir a modo de ejemplo: por una parte la praxis de la "convención nacional» desarrollada en muchos estados africanos, tiende a incluir en el proceso de democratización a los grupos de la sociedad civil en la medida más amplia posible; por la otra, en cambio, los pactos entre las élites se repiten con mayor frecuencia en las experiencias de los países latinoamericanos. 
En fin, con respecto a los sujetos protagonistas del proceso de transición y a la capacidad relativa para guiarlo o bien para participar en el mismo, un factor-clave debe identificarse en las modalidades seguidas por el proceso de formación de una nueva Constitución, que se puede configurar como producto y patrimonio de un sector de las fuerzas políticas o bien como resultado de un debate genuino nacional y como patrimonio del país en su conjunto. Ejemplos de los dos extremos de tal espectro nos lo ofrece la experiencia rusa (la Constitución de 1993 fue elaborada al interior del gabinete presidencial y después fue sometida a referéndum popular) y la experiencia de Uganda (la Constitución de 1996 fue objeto de debate y consulta entre los sectores de la población).

\section{La economía de mercado es una condición necesaria, pero no suficiente, de la democracia}

A tal conclusión se puede llegar, en particular, como consecuencia del análisis de las trayectorias evolutivas seguidas por los países de Europa centro-oriental y de Asia. La relación de causalidad que con frecuencia se instaura entre capitalismo y democracia, además de la contribución indiscutible que la introducción de una economía de mercado ha sido capaz de explicar en modo paralelo a la refundación democrática de una multiplicidad de ordenamientos constitucionales, en efecto no eximen para someter tal relación a una valoración más cuidadosa, con el fin de precisarla y de evidenciar, junto a las funciones positivas que se atribuyen a una economía de mercado, las potencialidades negativas de la misma, capaces por lo menos de manera tendencial, de minar el proceso de consolidación de la democracia.

Si se quiere analizar de manera individual los elementos positivos de la relación mencionada, se puede destacar, en primer lugar, el hecho que el mercado y la democracia parece que comparten la misma lógica antipaternalista, dejando al consumidor y al elector soberanos y jueces de sus propios intereses y haciendo que el éxito dependa, respectivamente, de las fuerzas políticas y de las empresas, del número de sujetos que alcancen a atraer hacia el propio producto en condiciones de libre competencia.

En segundo lugar, la economía de mercado se muestra en grado de favorecer la asignación del poder decisional y de otras formas de poder en sedes y esferas diversas de la estatal. Tal factor resulta funcional a la demo- 
cracia en una pluralidad de direcciones: facilita el desarrollo de una esfera autónoma de la "sociedad civil", que no es deudora del Estado por cuanto concierne a la disponibilidad de recursos, de informaciones y de capacidades de organización; tiende a restringir el aparato burocrático estatal; favorece la separación en esferas diversas de la competición por el poder económico y político.

Por otra parte, la operatividad del segundo de los elementos positivos citados, debe referirse no solo a economías planificadas de tipo soviético, sino también a formas de capitalismo controladas por el Estado. En efecto, si es evidente, el papel activo que el Estado posee en el ámbito del crecimiento económico en todas las fases del desarrollo capitalista, sin embargo es oportuno distinguir en tal contexto las hipótesis de intervención estatal de tipo simplemente complementario, de reglamentación y de manera eventual de correccón del mercado, de las hipótesis de intervención que llevan a que el Estado se sustituya al mercado y a que actúe como distribuidor principal de oportunidades económicas o bien como un sujeto que tiende a apropiarse en modo difuso del superávit económico. En efecto, las hipótesis planteadas por último producen — se nota tal fenómeno con referencia a los ordenamientos asiáticos- regímenes clientelistas y autoritarios, susceptibles de una democratización solo superficial y vulnerables, en la mayoría de los casos, frente a la difusión de los fenómenos endémicos de la corrupción.

Por otra parte, se configuran en modo no menos importante, las desventajas que puede producir la incontrolada introducción de la economía de mercado en correspondencia a la experimentación de las instituciones democráticas.

Las desigualdades sociales que han acompañado la liberalización de los mercados y la instauración de la libertad de competencia, la existencia amplia y difusa de los fenómenos de desocupación, las rápidas y frecuentemente impredecibles fluctuaciones de las economías de mercado, han convertido en unos casos significativos a los electores extremadamente vulnerables y súcubos de la movilización demagógica realizada, en los casos de especie, a favor y como soporte de las políticas autoritarias y de tipo exclusivista.

En los casos mencionados, el proceso de consolidación democrática aparece desprovisto de perspectivas de éxito positivo si no se encuentra acompañado de la creación de un sistema welfare y de democracia social capaz de proteger a las categorías más débiles o desfavorecidas de las vici- 
situdes del mercado y exige que las lógicas distintivas del Estado y el mercado sean reconocidas y preservadas de una erosión mutua.

\section{Las oportunidades de consolidación democrática resultan correlacionadas, aunque solo de manera tendencial, al desarrollo económico}

La ciencia política cuantitativa ha elaborado índices numéricos y estadísticos relacionados, respectivamente, con el proceso de democratización y de desarrollo económico y en serie de análisis estadística ha aplicado tales datos, entrecruzándolos, a un número de países bien amplio. Tal análisis, puesto en marcha desde hace tiempo por Lipset (en su ensayo "Some social requisites of democracy", en: American Political Science Review, 1959, p. 69 y ss.) y desarrollada en una pluralidad de contribuciones doctrinales sucesivas, entre las cuales no se pueden dejar de mencionar las de Axel Hadenius (ver la obra monográfica Democracy and Development, Cambridge, 1992 y el ensayo "The duration of Democracy: Institutional vs. Socioeconomic Factors", en: Beetham D. [coordinado por], Defining and Measuring Democracy, Sage Publications, 1995), ha llegado a varias conclusiones que son compartidas solo en parte: las perspectivas favorables para la consolidación democrática se sitúan en una relación directamente proporcional con relación al índice de desarrollo y al crecimiento de la economía.

Los motivos que inducen a observar una cierta cautela en relación al significado atribuído por los especialistas citados a los resultados de las propias investigaciones científicas coinciden en gran parte con los motivos de prudencia que, mencionados en el No 3 , sirven para circunscribir en modo amplio la configuración mecánica de la relación directa entre la consolidación democrática y la introducción de una economía de mercado. Por una parte, en tal contexto se puede destacar la existencia, de democracias subdesarrolladas $y$, por otra parte, de ordenamientos dotados de economías desarrolladas, pero que se caracterizan por tener un nivel de democratización modesto, déficit este que se puede notar, en particular, con referencia a la efectividad de las garantías de los derechos civiles y políticos (ejemplos de esto lo hallamos en un gran número de ordenamientos asiáticos). 


\section{Determinadas religiones son incompatibles con una sostenibilidad democrática plena}

La relación de congruencia unívoca entre protestantismo y democracia había recibido — como es sabido— una enunciación definitiva por parte de Max Weber: según tal tesis, el protestantismo, al incentivar una ética de responsabilidad personal, una vida asociativa rica y democrática y, por lo menos en sus variantes no conformistas, una separación clara entre Estado e Iglesia, proporcionó un terreno particularmente fértil para la democracia política.

La posición doctrinal aludida, que en tiempos recientes también ha encontrado otros seguidores respecto al momento de su enunciación (ver el ensayo de Lipset, "The Centrality of Political Culture", en: Journal of Democracy, 1990, p. 80 y ss.), es también destinada a una reexaminación profunda.

La naturaleza unidireccional y tendencialmente exclusivista de la posición misma, aparece en efecto circunstanciada en modo amplio por las transiciones democráticas que se verificaron en España y Portugal, países tradicionalmente católicos, por la experiencia de la teología de la liberación típica del catolicismo en América Latina, por la aptitud positiva de las jerarquías católicas eclesiásticas frente al proceso de consolidación democrática y del papel innegable que la Iglesia católica (y protestante) ha desarrollado en las fases de despegue de las transiciones constitucionales en algunos ordenamientos importantes de Europa centro-oriental (los ejemplos más significativos son la experiencia polaca y de Alemania oriental), contribuyendo en modo determinante a la realización del conocido efecto-dominó que se verificó con ocasión del desmantelamiento de los regímenes socialistas a partir de 1981, año del inicio de la lucha polaca por la libertad.

De otra parte, el credo ruso ortodoxo, el confucianismo y la fe islámica no parece que presenten características y contenidos que se encuentren en sintonía plena con las exigencias de desarrollo y consolidación de la democracia: el primero en cuanto privilegia una concepción de la voluntad popular trascendental más que empírica; el segundo en cuanto opta en favor de una subordinación clara del individuo al bien colectivo; la tercera en cuanto está caracterizada por una doctrina jurídica que no admite la separación entre el credo religioso y la política.

De todas formas en modo general, se muestra incompatible con la democracia cada forma de credo, de naturaleza espiritual o secular, que 
piense que tenga que reconducir la verdad final para la sociedad a un conocimiento superior y esotérico, al cual el no iniciado no puede tomar parte y al cual la autoridad política tiene que estar sujeta. Tal forma de credo parece constituir el presupuesto para la formación de derivas autoritarias y antidemocráticas, de entidad directamente proporcional con relación a los sectores de la sociedad que no comparten el mismo credo.

\section{La consolidación democrática encuentra serias dificultades en} presencia de sociedades articuladas y divididas en grupos culturales bien definidos y que son históricamente antagonistas

A mediados del siglo XIX John Stuart Mill había observado que las «instituciones libres son casi que imposibles en un país compuesto por diferentes nacionalidades», en cuanto cada persona teme más en las violaciones que se puedan causar por parte de las otras nacionalidades que aquellas causadas por parte del árbitro común, el Estado (en On Representative Government, cap. 16). El requisito de la unidad nacional, o bien la condición de una codivisión que sea pacífica y no conflictual — si bien en presencia de sociedades articuladas y complejas- de un común denominador mínimo de principios y valores fundamentales sobre los cuales se apoya cada ordenamiento y la observancia de reglas institucionales creadas, en primer lugar en sede constitucional, como criterio de ordenación de tal diversidad, representan efectivamente un background esencial en el ámbito del proceso de consolidación democrática.

Los ejemplos que pueden corroborar tal conclusión surgen copiosos: el proceso de transición constitucional y/o la fase sucesiva de consolidación democrática en efecto se han revelado particularmente arduos en las experiencias de los ordenamientos sub-saharianos, caracterizados por una fragmentación étnico-linguística extrema, a la cual se añade y se superpone la definición artificial de confines y territorios que se verificó con el ocaso del colonialismo; en algunas experiencias, con contornos a veces dramáticos, vividas en Europa centro-oriental y en los Balcanes (la referencia es, de manera obvia, a las transiciones ocurridas en el contexto de ex-Yugoslavia, pero también a las perturbaciones que han afectado y que todavía sacuden al "universo" que antes fue soviético, y que hoy es ruso y caucásico); en algunas experiencias asiáticas (conflictos étnicos y religiosos que han afectado a Indonesia, en particular en 1997) y, también latinoa- 
mericanas (un ejemplo, entre otros, nos lo ofrece el proceso de consolidación de la democracia en Guatemala, igualmente por la dificultad que muestran las minorías presentes en el territorio en aceptar los proyectos de reforma puestos en marcha).

\section{La forma de gobierno presidencial denota, con respecto a la} forma de gobierno parlamentaria, una funcionalidad menor para la consolidación democrática

En las fases de transición constitucional y de consolidación democrática, el "rendimiento» de las formas de gobierno parlamentarias por lo general se revela cualitativa y cuantitativamente mejor con relación a las formas de gobierno presidenciales. El número de poderes y de funciones de gran peso específico que se atribuyen normalmente al Jefe de Estado en las formas de gobierno presidenciales, factor al cual con frecuencia se acompaña un sistema político-partidista frágil o bien fragmentado, ha ocasionado derivas autoritarias de tipo presidencialista (de Vergottini) sobre todo en las repúblicas islámicas de la ex-URSS, en algunos ordenamientos latinoamericanos y en África, o bien de tipo populista en Asia y en algunos países latinoamericanos. Aparece radical el desvío del prototipo ofrecido por el modelo presidencial norteamericano, que en este sentido ha representado una imagen falsa y desorientadora de fácil y automática reproducción del modelo mismo en contextos que de manera evidente se encuentran poco preparados para acogerlo.

Las formas de gobierno parlamentarias, por el contrario, han reflejado una ductilidad y flexibilidad mayor, así como la capacidad de evitar o bien de amortiguar los conflictos que posiblemente surjan entre legislativo y ejecutivo: ejemplos de esto, los tenemos en modo particular, en algunas experiencias significativas de transición constitucional y de consolidación de la democracia (ya ocurrida) en los países de Europa centrooriental (República Checa, Hungría), pero también en varios ordenamientos asiáticos (Tailandia, Malasia).

En tal contexto, los sistemas electorales de tipo proporcional desarrollan una función política menos divisiva con respecto a los sistemas plurality, evidenciando así la propia capacidad para incentivar el compromiso entre los partidos y para la formación de un consenso, así como la creación de coaliciones de gobierno de gran alcance hasta lograr una unidad nacional. 
Factor este determinante en situaciones que vuelven implícita la aceptación en modo amplio de los procesos de cambio en acto, además de fluídas e inciertas en sus éxitos finales como son las de transición y de consolidación democrática.

\section{La sostenibilidad democrática aparece favorecida de manera} tendencial en los sistemas que adoptan un modelo federal o regional de repartición de poderes entre el ente soberano y los entes autónomos

Se trata de conclusiones a las cuales se puede llegar con referencia, en particular, a los ordenamientos en vía de transición/consolidación que se caracterizan por tener una división étnico-lingüística, racial y cultural evidente. En ese sentido, los sistemas que reconocen y garantizan, de acuerdo al modelo federal o regional, la existencia y el funcionamiento de las autonomías territoriales - constituyen ejemplos significativos, en el continente africano, el ordenamiento sudafricano, Nigeria y Etiopía; en el continente asiático, Malasia; en el continente latinoamericano, Brasil, Argentina y Venezuela — favorecen una multiplicación positiva y, en cierta medida, diferenciación a nivel local de los circuitos de dirección política que, al descentralizar el poder de decisión y al adelgazar el polo central, previenen o atenúan los impulsos y tendencias autoritarias y centralistas y valorizan las peculiaridades locales.

\section{El modelo constitucional de derivación liberal ha registrado una} afirmación solamente parcial

La exportación del modelo del constitucionalismo liberal en diversas partes del globo con relación a la realidad histórica-ideológica (Europa occidental y América del norte) que sirvió de cuna - prescindiendo de variantes a las cuales el constitucionalismo ha dado lugar desde el punto de vista histórico- ha encontrado dificultad y obstáculos en relación con los perfiles funcionales y procedimentales así como en lo que concierne a los contenidos axiológicos del constitucionalismo mismo.

A pesar que nos limitamos a considerar la adopción de los perfiles meramente funcionales/procedimentales del constitucionalismo - prin- 
cipio de la soberanía popular, principio de mayorías para adoptar decisiones políticas, principio de separación de poderes, control de constitucionalidad de las leyes etc.- - Semejante adopción se ha manifestado en muchos de los ordenamientos examinados, de acuerdo a la evaluación de los hechos, unicamente formal, superficial o bien priva de efectividad, en cuanto se ha concebido como un fenómeno de implantación de procedimientos e instrumentos en contextos culturales e institucionales que aún no están preparados para garantizar un funcionamiento adecuado. Si la investigación se extiende a la adopción de contenidos axiológicos del constitucionalismo liberal, nos percatamos del hecho que la consolidación parcial del mismo deriva también de la incapacidad de asegurar, manifestada en algunos ordenamientos, más allá de la adopción del modelo liberal, la garantía de los derechos de la persona, valores esenciales en la concepción liberal del Estado y de la Constitución. De otra parte, los tentativos por trasplantar el modelo constitucional de derivación liberal han dado lugar, en un gran número de hipótesis, a fenómenos de rechazo en cuanto son efectuados en presencia de valores constitucionales que son incompatibles con los liberales, que han llevado al fracaso de los tentativos mismos.

«Esto significa que ciertas aserciones sobre el éxito de la democratización, que hacen parte de los perfiles del gran fenómeno de la globalización, tienden a revelarse fruto de observaciones superficiales, que son desmentidas por los hechos» (de Vergottini). El constitucionalismo que, si bien referido a aquellos ordenamientos que habían sido la sede de elaboración y formación, aparece cercano al cumplimiento de la misión histórica que le ha sido asignada y que es llamado a medirse de acuerdo a los nuevos desafíos impuestos por el proceso de globalización, todavía parece que se mueve y opera, en primer lugar bajo el aspecto instrumentalprocedimental, a un nivel embrionario y primordial para la mayoría de los ordenamientos que experimentan procesos de transición, y aún es largo y lleno de trampas e incertidumbres el camino que le queda por recorrer.

De otra parte, bajo el aspecto de contenido y axiológico, si es fácil suponer la imposibilidad de remover, a corto y mediano plazo, los obstáculos de naturaleza cultural y político-institucional que hasta ahora se han interpuesto a la adopción de una mesa de valores liberal-democráticos por las experiencias tomadas en consideración, también a largo plazo es inevitable que estas experiencias se conviertan en la sede de aplicación, que a 
esta altura se difunde a nivel global como expresión de aceptación de los valores que son comunes a la humanidad entera, de los mismos principios constitucionales que se encuentran vigentes en las viejas democracias nacionales (derechos del hombre - internacionalizados y globalizados - y soberanía popular), en la conciencia plena que la universalización de los derechos fundamentales no impide la expresión de las diferentes culturas, sino que constituye una condición imprescindible para su legitimación, equiparación y protección jurídica.

La instauración de "un orden mundial nuevo» de acuerdo a las crismas de la democracia liberal y a la realización de una República universal fundada sobre los pricipios de justicia, de subsidiariedad y del federalismo y afirmada por una teoría de las virtudes civiles universales, enunciada a finales del siglo XX por una doctrina filosófica acreditada (Höffe), aparecen - al alba del siglo XXI- objetivos que aún no se encuentran en el horizonte; la actualidad de las funciones explicables por el constitucionalismo todavía aparece en su evidencia plena. 